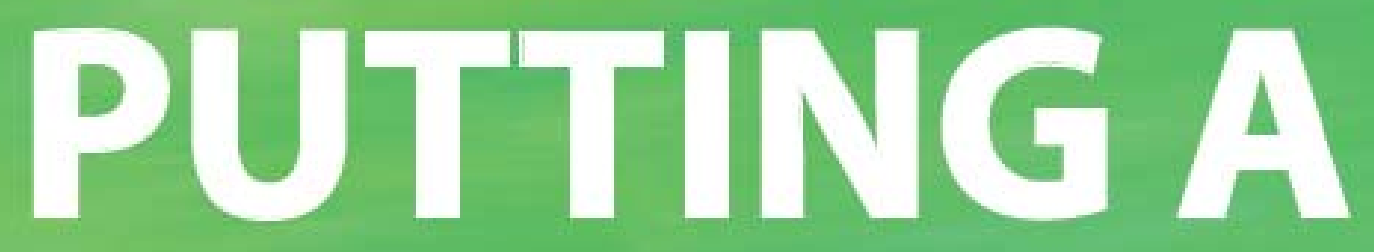

SPIN ON

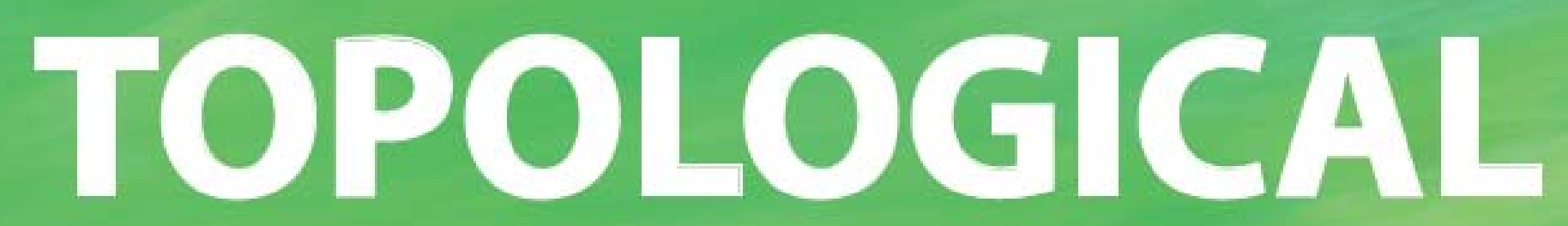

MATTER

Joris Voerman 


\section{PUTTING A SPIN ON \\ TOPOLOGICAL}

MATTER

Joris Voerman 



\title{
PUTTING A SPIN ON TOPOLOGICAL MATTER
}

\section{DISSERTATION}

\author{
to obtain
}

the degree of doctor at the University of Twente,

on the authority of the Rector Magnificus,

prof. dr. T.T.M. Palstra,

on account of the decision of the doctorate board,

to be publicly defended

on thursday the $18^{\text {th }}$ of April, 2019, at 12:45

by

Joris Anthonie Voerman

born on the $25^{\text {th }}$ of May, 1992

in Nieuwegein, the Netherlands 
This dissertation has been approved by:

\section{Promotor:}

prof. dr. ir. A. Brinkman

And co-promotor:

dr. C. Li 


\section{Doctorate Board:}

Chair and secretary:

prof. dr. J.L. Herek University of Twente

Promotor:

prof. dr. ir. A. Brinkman University of Twente

Co-promotor:

dr. C. Li University of Twente

Members:

prof. dr. I. Adagideli

prof. dr. ir. M. Huijben

Sabanci University, Istanbul

dr. ir. M. P. de Jong

University of Twente

University of Twente

prof. dr. ir. H. J. W. Zandvliet

University of Twente

prof. dr. U. Zeitler

HFML, Radboud University

The work described in this thesis was carried out in the Quantum Transport in Matter group, MESA+ Institute for Nanotechnology, University of Twente, the Netherlands.

This work was financially supported by the European Research Council (ERC) through the ERC Consolidator grant.

Putting a spin on topological matter

$\mathrm{PhD}$ Thesis, University of Twente

Printed by: GildePrint Drukkerijen, Enschede, the Netherlands

ISBN: 978-90-365-4751-2

DOI: $10.3990 / 1.9789036547512$ 


\section{Contents}

$\begin{array}{lll}1 & \text { Introduction } & 7\end{array}$

1.1 Introduction . . . . . . . . . . . . . . 8

1.2 Spin .................... . . . 8

1.3 Topological insulators ................. . . 9

1.4 Spin in a topological insulator . . . . . . . . . . . . . . . . . 13

1.5 Spin in a superconductor ................ 14

1.6 Spintronics . . . . . . . . . . . . . . . . 17

1.7 Outline of the thesis .................. 19

2 Materials and Methods $\quad 21$

2.1 Spin-momentum locking in BSTS . . . . . . . . . . . 22

2.2 ZrSiS Crystals . . . . . . . . . . . . . . . 28

$2.3 \mathrm{PdTe}_{2}$ superconducting junctions . . . . . . . . . . . 31

3 Spin-momentum locking in the gate tunable topological insulator BiSbTeSe 2 in non-local transport measurements 35

3.1 Introduction . . . . . . . . . . . . . . . . 36

3.2 Proof of principle . . . . . . . . . . . . . . . . . . 37

3.3 Tuning the Fermi energy . . . . . . . . . . . . . . . . 40

3.4 Discussion . . . . . . . . . . . . . . . . . . 42

3.5 Conclusions and outlook . . . . . . . . . . . . 46

4 Upper limit to the energy retention in the $3 \mathrm{D}$ topological $\begin{array}{lr}\text { insulator BiSbTeSe } & 47\end{array}$

4.1 Introduction . . . . . . . . . . . . . . . . . . 48

4.2 DC Measurements . . . . . . . . . . . . . . . . 49

4.3 AC Measurements ................. 51

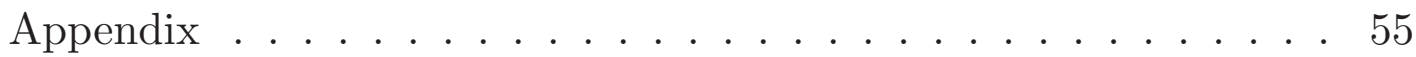

5 Origin of the butterfly magnetoresistance in $\mathrm{ZrSiS} \quad 57$

5.1 Introduction . . . . . . . . . . . . . . 58

5.2 Butterfly magnetoresistance . . . . . . . . . . . . 58

5.3 Shubnikov-de Haas oscillations . . . . . . . . . . . 63 
5.4 The Lifshitz-Kosevich model . . . . . . . . . . . . . . . . . 64

5.5 Conclusions . . . . . . . . . . . . . . . 70

Appendix ......................... 71

6 S-wave superconductivity in $\mathrm{PdTe}_{2}$ observed by tunneling spectroscopy on side-junctions $\quad 73$

6.1 Introduction . . . . . . . . . . . . . . . . 74

6.2 The experiment . . . . . . . . . . . . . . 74

6.3 The BTK Model . . . . . . . . . . . . . . . . . 76

6.4 The $\mathrm{I}_{c}+$ BTK Model . . . . . . . . . . . . . . . . . 79

6.5 Additional features . . . . . . . . . . . . . . . . 80

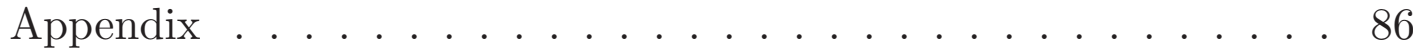

$\begin{array}{ll}\text { Summary } & 88\end{array}$

$\begin{array}{ll}\text { Samenvatting } & 91\end{array}$

$\begin{array}{ll}\text { Dankwoord } & 95\end{array}$

$\begin{array}{ll}\text { Bibliography } & 99\end{array}$ 



\section{Introduction}

Spintronics is heralded as the next step in integrated circuit technology beyond the current semiconductor technology. This chapter deals with the fundamental concepts of spin and the role that the electron spin plays in topological insulators and topological superconductors. The road taken and ahead of spintronics is briefly described, followed by an outline of the thesis. 


\subsection{Introduction}

Since the Nobel-prize-winning invention of the transistor in 1947 by Shockley, Bardeen, and Brattain, society has undergone some incredible changes that have had a major influence on our daily lives [1]. The past fifty years have seen the advent of the personal computer as well as the invention and virus-like spread of the world wide web. The miniaturization of transistors have led to the omnipresence of smartphones. A technology that has taken the market by storm and has permanently ingrained itself in the lives of many. Computers and related devices have become faster and more powerful year by year, because the individual transistors that make up the integrated circuits have shrunk in size. Simply put, every year we can put more transistors in the same area. Leading chip manufacturers now mass-produce transistors that are only $14 \mathrm{~nm}$ in size [2]. Obviously this continuous shrinking must come to a hold somewhere. Typical interatomic distances are on the order of several Angstrom, but before this limit is reached, the gate dielectric, that electronically separates the gate channel from the source and the drain, becomes too thin to effectively stop electrons from passing through it. Combined with the increased heat dissipated by more and more transistors per area, this shrinking-limit calls for a revolutionary new way of computing [3-6]. Conventional electronics only uses the charge of the electron for computations. Transistors switch between no conduction between the source (S) and drain (D), a logic " 0 ", and the presence of conduction, a logic "1". Electrons do have another property besides a charge of 1.6 . $10^{-19} \mathrm{C}$, that we might make use of: the intrinsic magnetic moment called "spin". Being able to manipulate and detect the electron spin can open up a wide range of computational applications $[7,8]$.

\section{$1.2 \quad$ Spin}

Electron spin is sometimes described as the magnetic moment that is associated with the circulating outer shell of the electron, similar to the magnetic moment of a coiled wire. This explanation draws parallels with the classical picture of angular momentum, e.g. the rotation of the earth, hence the term spin. Although this notion is simple to understand it is, unfortunately, also 
unphysical ${ }^{1}$. Nevertheless, all elementary particles have their own intrinsic magnetic moment, which we call spin. The value of the spin can be either integer or half integer, which separates all elementary particles in two distinct categories: spin integer bosons and spin half-integer fermions [9]. Electrons with $\operatorname{spin}^{2} 1 / 2$ are thus fermions, which means that upon exchanging the position of two electrons in the same quantum state, the wavefunction picks up a minus sign. When we now consider two electrons that share the same location and we take the minus sign into account, the resulting value of the wavefunction is zero. They cannot exist, or as physicists say: electrons obey the Pauli exclusion principle. Bosons, on the other hand, have no qualms about being in the same quantum state. Because the electron spin is $1 / 2$ electrons can come in pairs. Every state can be occupied by a $-1 / 2$ (spin down) and a $+1 / 2$ (spin up) electron, which are the only two flavors of electrons possible. A feature that will prove highly relevant throughout this thesis.

\subsection{Topological insulators}

In condensed matter physics we call a material an insulator when there is an absence of states within a large region $(\sim 100 \mathrm{meV})$ around the Fermi energy $\left(\mathrm{E}_{F}\right)$ for all possible electron momenta $k$. Figure 1.1(a) schemati-

\footnotetext{
${ }^{1}$ When we treat the electron as a point on its surface with charge $e$ that rotates, the current of this rotation for electron radius $R$ is given by:

$$
I=\frac{e \cdot v}{2 \pi R}
$$

where $v$ is the velocity of a point on the surface. For a circulating current we know that the magnetic moment $\mu=I \cdot A$, where $A$ is the area of the loop. The intrinsic magnetic moment of an electron is roughly equal to the Bohr magneton, $\mu_{B}$, so we can equate these two magnetic moments. When we put in the classical radius of an electron, $R_{e}=2.82 \cdot 10^{-15} \mathrm{~m}$, we find:

$$
\mu_{B}=\frac{e \cdot v}{2 \pi R} \pi R^{2}=\frac{e \cdot v \cdot R}{2}
$$

from which it follows that

$$
v=\frac{2 \mu_{B}}{e R}=4.1 \cdot 10^{10} \mathrm{~m} / \mathrm{s}
$$

This value is more than one hundred times larger than the speed of light. Not a very physical interpretation of spin indeed!

${ }^{2}$ In units of the reduced Planck constant $\hbar$, which will be omitted in this thesis
} 
cally shows this situation, where there is a valence band (VB) below $\mathrm{E}_{F}$, filled with electrons and a conduction band $(\mathrm{CB})$ above $\mathrm{E}_{F}$, devoid of electrons. For an electron to be accelerated in an electric field it would need to obtain a higher energy. Since there are no energy states available for it, it cannot do so. Insulators do not conduct electricity, as we know. For other materials the bands lie closer together, so that the thermal smearing, given by the Fermi-Dirac distribution, creates a small, but non-zero filling of the conduction band. Now there are a few electrons available for transport. These materials are known as semiconductors and are the workhorse materials in the computer industry right now, because they require only a small push to bump them out of this conducting state. When the Fermi energy crosses a band we call the material a metal. There is an abundance of available states and many electrons can participate in charge transport. Copper and gold are examples of metals used for their excellent electronic transport properties.

The energy bands that are so crucial for the electronic transport properties of the material are formed from atomic orbitals. For a single atom the different orbitals (often called "shells") have well-defined and separated energies, but in a periodic lattice these orbitals merge together and form a sea of possible energy states [10]. Solely based on these orbital energies there is an ordering of the bands in energy, 2s comes after 1s for example. Upon closer inspection there are other mechanisms that can alter the energy of a band, most notably spin-orbit coupling (SOC). Although SOC occurs in every system, it is most pronounced in larger atoms, since their outer shells have larger orbital angular momenta. The different spins in the bands each feel an opposite SOC. The energy bands move closer to each, one going to higher energies and the other to lower energies. For most crystals this has little effect, but in some cases the upwards moving orbital ends up higher than the downwards moving orbital, as indicated in figure 1.1(b). The band structure of such a material can still have an energy gap because the two crossing bands feel each other's presence and hybridize. We call the resulting gap an "inverted gap", but it still does not conduct electricity. Once we couple our inverted gap material to a regular insulator ${ }^{3}$ something curious happens at the interface. Because the orbitals, and thus the bands, are orthogonal states, the VB on one side cannot couple to the VB on the other side. It can only continue from the $\mathrm{VB}$ of one side to the $\mathrm{CB}$ of the other side. The end product is a surface state (SS) that has to span the

\footnotetext{
${ }^{3}$ This can be air or vacuum too
} 


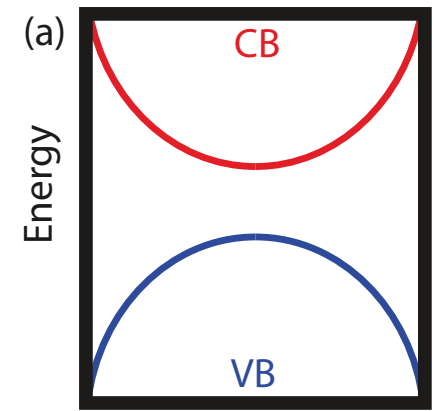

Momentum

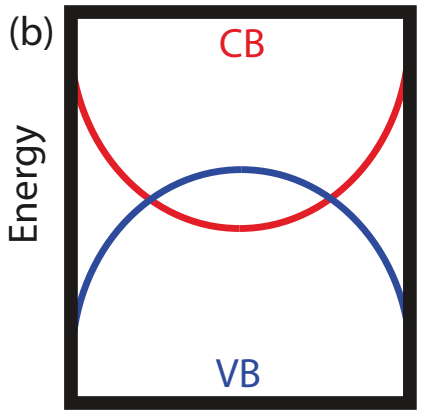

Momentum

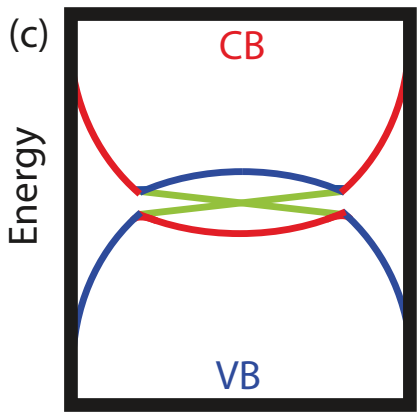

Momentum

Figure 1.1: Schematic band structures illustrating the origin of a topological surface state. (a) Schematic band structure of a trivial insulator. The red (blue) line indicates the bulk conduction (valence) band, labeled CB (VB). (b) SOC shifts the two orbitals closer to each other and in this case even crosses them. (c) The two orbitals hybridize into a new CB and VB, forming a TI. When the TI is connected to a trivial insulator a linear surface state appears, shown in green.

bandgap and cross the Fermi level, and thus the outer edge of the inverted gap material can conduct electricity. Figure 1.1(c) shows this is peculiar state of matter, that is called a topological insulator (TI).

\section{Weyl semimetals}

Only a few years after the discovery of TIs the collection of topological materials was expanded with Weyl semimetals (WSM) [11-16], named after the German mathematician Hermann Weyl who showed the possibility of the existence of massless fermions from the Dirac equation [17]. These massless Weyl fermions were first found in TaAs [18] and are characterized by linearly dispersing states in the Brillouin zone (BZ). In broad strokes, this is the $3 \mathrm{D}$ equivalent of a TI. TIs have linearly dispersing states, a 2D cone, in the surface BZ, whereas a WSM has a 3D cone in the bulk BZ. Generally, one could argue that the WSM is then the surface of a 4D material, but this seems rather complacent and raises more questions than it answers. The TI, however, is a good starting point towards understanding WSMs. In a TI the bands invert because of SOC and open a hybridization gap across the BZ, which must be spanned by a surface state because the crystal is finite ${ }^{4}$ and the inverted gap couples to a non-inverted gap. Now imagine that this TI is the BZ of a larger crystal. Somewhere inside this BZ there is an inverted gap that is connected to a non-inverted gap, already within the BZ or in the neighboring BZ. This would mean that there is a

\footnotetext{
${ }^{4}$ In technical terms the surface of the crystal is where the inversion symmetry breaks
} 
topologically protected linear state already in the bulk of the material: a WSM. This picture is, in fact, equal to figure 1.1(b). The difference between the TI and the WSM is that in the case of the WSM there is one direction in momentum space where the two inverted bands do not open a gap. In almost every cut through the BZ the dispersion will look like figure 1.1(c), but in one direction the bands are fully orthogonal and they remain crossed maintaining the dispersion of figure 1.1(b). From this drawing we can see that this crossing must always occur at two points in the BZ and in fact these so-called Weyl points (WPs) always come in pairs of two. Moving the two WPs in momentum space is analogous to moving the bands further away from each other or towards each other, since the WPs are positioned where the bands cross. From figure 1.1(b) we can deduce this relation between the WP position and the energy difference between the two bands. When the WPs move closer until they touch the system becomes trivial again. We now have the situation as described by figure 1.1(a), just with an infinitesimally small gap between the VB and CB [19].

Based on the picture of the SOC moving the bands closer to each other, but not splitting the spins, one would expect each of the WPs to be twofold degenerate in spin. Materials where this occurs are called Dirac semimetals (DSMs) and thus have four Dirac points in their BZ. Applying a magnetic field, for example, can lift this degeneracy. DSMs are slightly more common than WSMs and were predicted before WSMs [20, 21]. The workhorse WSM nowadays is TaAs $[14,15]$. Commonly used Dirac semimetals include $\mathrm{Cd}_{3} \mathrm{As}_{2}$ [22], $\mathrm{Na} 3 \mathrm{Bi}[23]$ and perfectly tuned $\mathrm{Bi}_{1-x} \mathrm{Sb}_{x}$, where $x$ is around $3 \%$ [24]. In this case the VB and CB just touch each other forming a Dirac cone more or less by accident. It is clear that this case is different from the robust WSM described earlier. In this thesis the Dirac semimetals $\mathrm{PdTe}_{2}$ (chapter 6) and $\mathrm{ZrSiS}$ (chapter 5) are used. Chapter 2 provides more information on the specific details of these crystals. ZrSiS is not commonly called a DSM or WSM, but a nodal line semimetal. As we have seen, a TI is gapped in all directions, a WSM has one direction in which there is a crossing at the Fermi level yielding two Dirac points. In a nodal line semimetal there is only one direction that is gapped, which results in a circular line of Dirac points [25]. 


\subsection{Spin in a topological insulator}

Electron spin plays a crucial role in the transport properties of the topological surface state. To understand what the effect of the electron spin is, the concept of a topological insulator can be built up from another starting point than that of section 1.3. When a current flows in an out of plane magnetic field the charge carriers experience a Lorentz force that drives the electrons to one side and holes to the other side, creating a voltage difference. This phenomenon was discovered by Edward Hall in 1987 and subsequently named the Hall effect [26]. Performing this experiment on a ferromagnetic sample means that the magnetic field will not only create a Lorentz force, but also magnetize the sample. Even when the field is then put to zero, the Hall effect remains because of the internal magnetization of the sample. This anomalous Hall effect (AHE) was first noticed a few years later by Hall and more thoroughly understood by Karplus and Luttinger [27-29]. In 1980 Klaus von Klitzing discovered that in his small devices the Hall resistance was exactly quantized, a discovery for which he won the Nobel prize five years later $[30,31]$. This quantum Hall effect (QHE) can be understood in terms of Landau levels (LL). Highly mobile charge carriers can be forced into a circular motion by the magnetic field as drawn in figure 1.2(a). This means that this carrier is no longer available for transport and actually forms something that is similar to an atom, since electrons also occupy circular orbits. Just as is the case for atoms the energy here is quantized and the resulting energy levels are called Landau levels. At the edge of the material the orbits cannot close, causing the electrons to only move along the edge in a hopping fashion.

In TIs a combination of these phenomena occurs. The charge carriers are forced into Landau levels even in the absence of an external magnetic field. The role of the magnetic field is in this case played by SOC, which makes it quite different from the AHE. In this case the effective magnetic field depends on the spin of the electron and is thus opposite for spin up and spin down electrons, which is why TIs are also known as quantum spin Hall insulators (QSHI) [32-35]. The 1D conducting edge channels that form in a $2 \mathrm{D}$ TI thus have the momentum of the electron coupled to the spin, as indicated in figure 1.2(b). This property of TIs is called spinmomentum locking (SML) and is what makes TIs such a desirable class of materials for spintronic applications. Simply running a charge current through a TI gives a spin polarization, making the TI an effective spingenerator. Once the spin polarization is formed, or spins are injected into a 
(a)

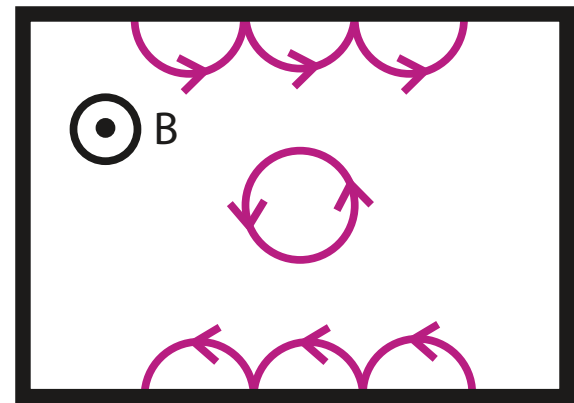

(b)

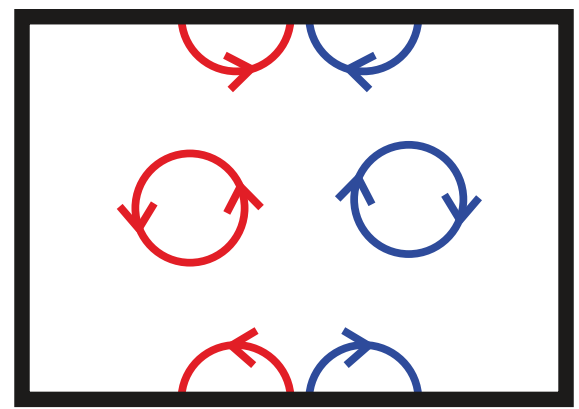

Figure 1.2: (a) quantum Hall insulator. An out of plane magnetic field forces electrons into circular orbits (LLs). At the edge the incomplete orbits provide transport in a hopping motion. The purple color indicates that this effect is the same for both electron spins. (b) quantum spin Hall insulator. SOC takes the role of the magnetic field forcing the electrons into LLs. Red and blue represent up and down electrons and have opposite rotation leading to spin-momentum locking on the edges.

TI, this information is very well protected. Because the spin and momentum are coupled, $180^{\circ}$ backscattering is prohibited in a $2 \mathrm{D}$ TI and is the only possible scattering direction in the 1D edge state. Flipping the spin and thus losing the information, requires an electron to move to the other edge of the TI. TIs can thus be used to transport spin information over distances longer than what is possible in other materials, even graphene that is known for its long spin relaxation length on the order of microns [36, 37]. The fact that a dissipationless current can run in the edge state of an ideal 2D TI could solve problems regarding the enormous heat dissipation that plagues current semiconductor technology. It should be noted however, that in the $2 \mathrm{D}$ surface of a 3D TI there are many scattering directions that are still allowed, even though $180^{\circ}$ is prohibited. A 3D TI will therefore still have to deal with heat dissipation.

\subsection{Spin in a superconductor}

In a superconductor the current is not carried by electrons, but by pairs of electrons known as Cooper pairs [38]. The theory of conventional superconductivity was first described by Bardeen, Cooper, and Schrieffer in 1957, who later earned a Nobel prize for their work, and is called BCS theory after the inventors [39]. In BCS theory two electrons from the same orbital, but with opposite momentum, couple to each other by means of a phonon. The coupled states are no longer bound to the Pauli principle and are moved to zero energy, leaving a band in energy devoid of states, 
called the superconducting gap $\Delta$. The mechanism that pairs the electrons in Cooper pairs is known as the order parameter (OP) and since we are dealing with a 2 -fermion wavefunction the OP must be anti-symmetric. Because the states share an orbital this part of the OP is symmetric, meaning that conventional Cooper pairs have an antisymmetric spin part of the OP. These Cooper pairs thus form a spin 0 singlet and behave as bosons, which allows all Cooper pairs to share the same wavefunction. The shape of the gap $\Delta$ in momentum space looks like an atomic s-orbital, which is why this conventional form of pairing is known as $s$-wave pairing.

Although the exact mechanisms remain unknown to this day, it is possible to form Cooper pairs through other pairing mechanisms. All superconductivity that cannot be explained by BCS theory is collectively known as unconventional superconductivity $[40,41]$. In these materials the two spins can be coupled differently, for example the spin-triplet state is noted as the most likely spin state of the Cooper pairs in $\mathrm{Sr}_{2} \mathrm{RuO}_{4}$ [42, 43]. Furthermore, the spatial configuration of the $\mathrm{OP}$ is not necessarily spatially homogeneous. The shape of the OP and the gap $\Delta$ can in principle take the shape of any orbital. The high $\mathrm{T}_{c}$ superconducting cuprate $\mathrm{YBa}_{2} \mathrm{Cu}_{3} \mathrm{O}_{7}$ (YBCO), for example, exhibits d-wave symmetry pairing in the OP [44].

\section{Andreev reflections}

Imagine an electron in a normal metal, that is connected to a superconductor. When the electron reaches the interface it cannot simply continue into the superconductor where the current is carried by Cooper pairs. Only when the electron has an energy equal to or greater than $\Delta$ can it enter as an electron. Instead, there are two possibilities for our electron: it can simply reflect like any electron at a potential barrier would, or it can reflect as a hole. The latter reflection is accompanied by a charge transfer of 2e, the charge of a Cooper pair. By reflecting as a hole, the electron can continue into the superconductor as a Cooper pair as illustrated by figure 1.3; a process known as Andreev reflection [45, 46]. These reflections can, through interference of the incoming and reflected wave, form bound states known as Andreev bound states (ABS). The ABS can sit at any energy below $\Delta$, even at zero energy. In this situation the ABS has some unusual properties. Like all ABS it is half-hole and half-electron, but when it has zero energy it fulfills the criteria of a Majorana fermion: A particle that is its own antiparticle [47]. We should not forget that it is not a particle, but 
(a)

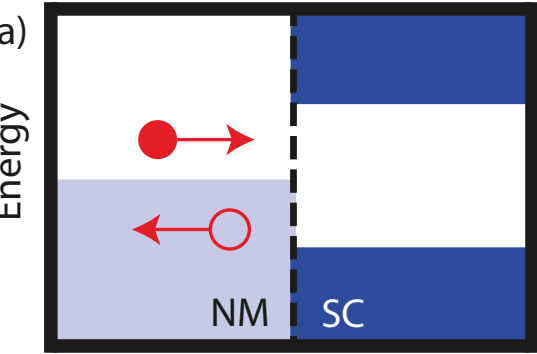

Place

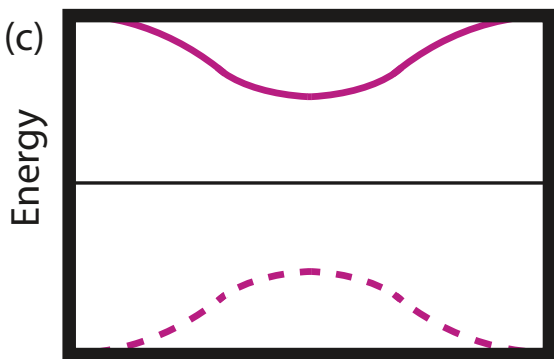

Phase

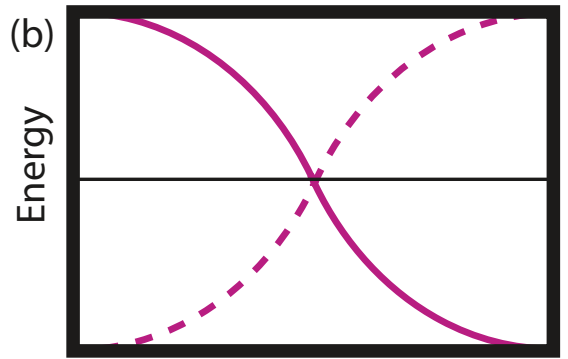

Phase

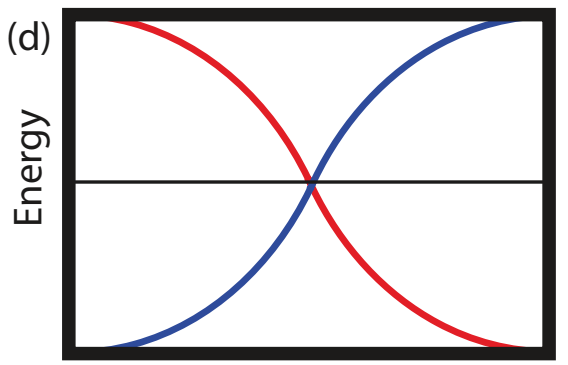

Phase

Figure 1.3: (a) Illustration of an NS interface. An electron (red closed circle) reflects as a hole (open circle) at the interface since it can enter the superconductor only as a Cooper pair. (b) Schematic energy-phase diagram of the ABSs before taking hybridization into account. The positive and negative energy mode are drawn as a solid and dotted line. The purple color indicates that both spin species are equally present. (c) The two ABSs have hybridized and formed a gap. The ABS at zero energy (the MBS) has disappeared. (d) The two ABS branches now belong to one spin, making them orthogonal. Because they can no longer hybridize the MBS remains.

a bound state and so it is called a Majorana bound state (MBS). Having experimental control over these MBS is one of the hottest topics in condensed matter physics right now and would be a huge boost to the field of quantum computing $[48,49]$.

Unfortunately, obtaining a MBS is not as simple as it was described just now. There is a finite coupling possible between the two branches of the Andreev bound state, which means that they hybridize and open a gap in energy, as drawn in figure 1.3(c). As long as these two branches are not orthogonal there will not be a MBS.If each branch were to only have one spin state, the two branches would be orthogonal and could no longer hybridize, thus preserving the MBS. This is also a sort of spin-momentum locking (SML), but of the Cooper pairs instead of electrons as in section 1.4, so it is referred to as topological superconductivity. This situation, as 
described in figure 1.3(d) can be obtained by either taking a material that exhibits SML and proximizing it with a superconductor, or finding a material that has both of these properties intrinsically: a topological superconductor. Scientists have found numerous materials that host topological states with SML, but topological superconductors are rare and highly sought-after $[50,52]$. Chapter 6 describes experiments investigating the nature of the superconductivity of $\mathrm{PdTe}_{2}$, which is a prime candidate for being a topological superconductor.

\subsection{Spintronics}

The field of spintronics aims to replace or improve existing semiconductor technology with the use of phenomena that relate to the electron spin $[5,8]$. Spintronic elements typically make use of one of two closely related effects: tunneling magnetoresistance (TMR) and giant magnetoresistance (GMR). A TMR structure consists of two ferromagnetic layers separated by an insulating layer. The total resistance of this stack depends on the alignment of the magnetization of the two ferromagnetic layers, as was first found by Jullière [53]. Later, the insulating layer was replaced by a normal metal, in which case it is called GMR. The simultaneous discovery of this effect by Fert and Grünberg was awarded with a Nobel prize in 2007 [54-56]. Both effects rely on the fact that tunneling into a ferromagnet (FM) depends on the number of available states to tunnel into. In a normal metal this number is equal for both electron spins, but in an FM there is a different number of states available for the two electron spins. Figure 1.4 illustrates the TMR effect. A parallel alignment of the ferromagnetic layers means that there are many electrons and many states available to tunnel into, which gives a low resistance. Antiparallel alignment of the FM layers means that the majority spins have only few states to tunnel into, increasing the resistance.

Current magnetoresistive RAM (MRAM) technology uses this effect as a way of storing information. A current running through a small wire has a magnetic field around it, which changes the magnetization of one of the ferromagnetic layers, thus switching it from a 1 to a 0 or vice versa. Even when the current is removed the magnetization will remain, a desirable memory feature called non-volatility. Scaling down MRAM further than its current state is hampered by stray magnetic fields. Shrinking down the bits and putting them closer means that the magnetic fields coming from the wires will extend over multiple bits and they will influence each other greatly. 
(a)

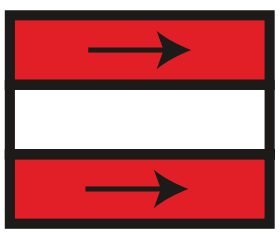

(c)

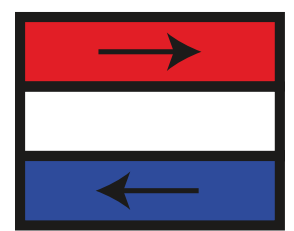

(b)

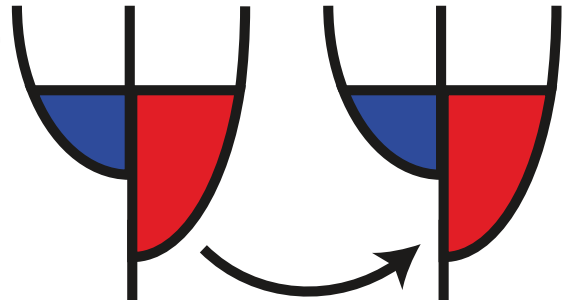

(d)

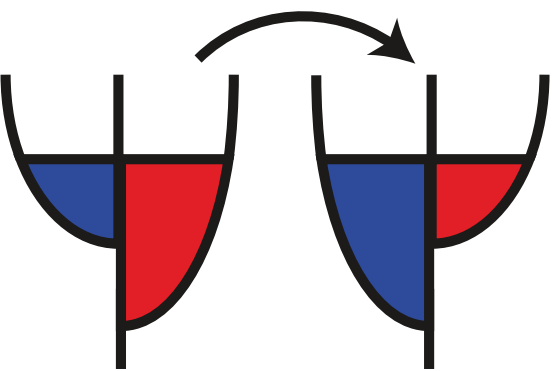

Figure 1.4: (a) Schematic of a TMR device in parallel configuration. Red represents positive magnetization of the FM layer, as indicated by the arrows. The whitespace shows the insulating middle layer. (b) Energy diagram of the tunneling. There are a lot of states available on the right for the electrons coming from the red part on the left. This is the low resistance state. (c) The TMR device in antiparallel configuration. Blue represents the negative magnetization as indicated by the arrow. (d) There are few states on the right for the red electrons coming from the left. This is the high resistance state.

(a)

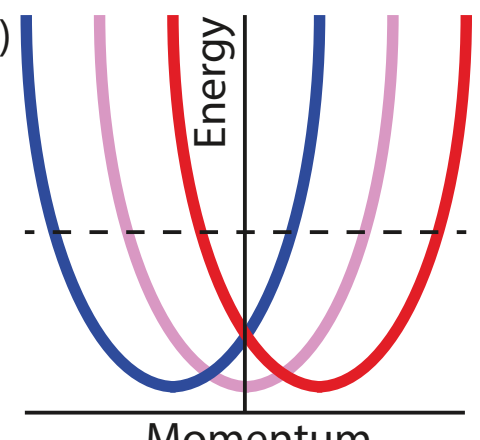

(b)

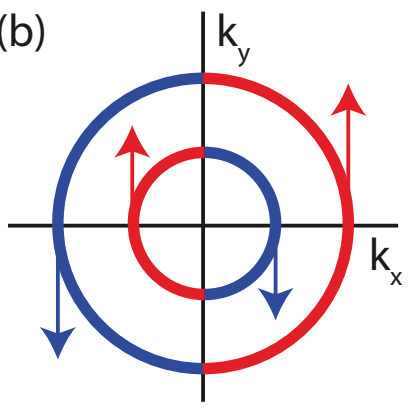

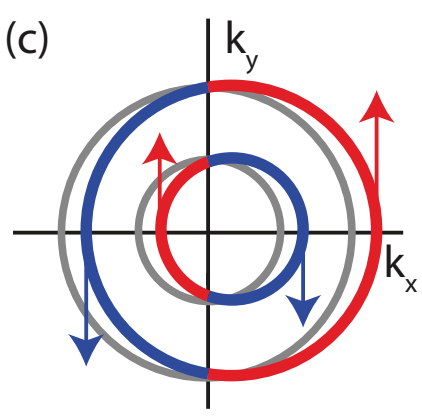

Figure 1.5: Illustration of the direct Rashba-Edelstein effect. (a) The Rashba effect splits a spin-degenerate band (pale purple) into two bands according to their spin (red and blue). The dotted line represents the Fermi energy. (b) The Fermi surface seen in the $\mathrm{k}_{x}-\mathrm{k}_{y}$ plane. The two concentric circles each have their spin locked to their momentum. The colors are derived from (a) to remind the reader of the spin texture. (c) An applied electric field shifts both circles in one direction. There is now more red than blue in the graph indicating a net spin-polarization has been created by the DREE. 
The field of spintronics has great interest in spin-transfer torque (STT), which would allow further miniaturization of magnetic memory elements [57]. STT is the effect by which an electric current running parallel to a FM layer can influence the magnetization of this layer. When an electron enters the FM, but the spin does not nicely match the magnetization it will slightly disturb the magnetization $[58,59]$. There are several physical phenomena that can generate a spin polarized current that in turn can be used to create STT as efficiently as possible. First of all, the spin-Hall effect (SHE), which was predicted by Dyakonov and Perel in 1971 [60, 61] and experimentally observed in 2004 [62,63]. Because of the spin-orbit interaction a charge current will be split in spin perpendicularly, analogous to the Hall effect which splits charge perpendicular to the current direction by a magnetic field. For some applications the spin-splitting perpendicular to the current is not ideal. The direct Rashba-Edelstein effect (DREE) is similar to the SHE in that it creates a spin-accumulation from a charge current, but it does so without physically separating the spins in the crystal [64-66]. The Rashba SOC splits the two spin types in a degenerate band in momentum (figure 1.5(a)). The Fermi surface then looks like two concentric cirles, each with their own spin-momentum locking, as illustrated in figure 1.5(b). An applied electric field shifts these circles slightly in one direction, which gives the current a spin-polarization, as shown in figure 1.5(c). Finally, the topological surface state (TSS) generates a spin-polarization from an applied electric field in the same way, but generally more efficiently. The Fermi surface in the TSS lacks one of circles, because that circle is moved to the other side of the crystal. In the DREE the two Fermi circles generate opposing spin-polarizations, but in the case of a TSS there is no Fermi circle to do so. This makes the TI one of the most promising ways of creating a large spin-polarized current and thus a large STT, as has been shown experimentally [67, 68].

\subsection{Outline of the thesis}

Chapter 2 delves deeper into the methods and materials that are used throughout this thesis. It explains how the nanodevices are made and what design choices are made in the run-up to the experiments themselves. We will study the origin and structure of some crystals that are in high demand in condensed matter physics for their extraordinary properties. The most notable material is $\mathrm{BiSbTeSe}_{2}$, which is the result of years of studies into 
perfecting a 3D TI.

In Chapter 3 I show the results of experiments probing the SML in $\mathrm{BiSbTeSe}_{2}$ (BSTS), a TI that has very little contribution of the bulk states to the transport properties. Electrons are spin polarized by sending a current through a crystalline flake of BSTS. Using ferromagnetic contacts I probe the spin polarization of the electrons in a non-local configuration. We will see that SML of the TSS is verified experimentally, allowing us to research the effect of gating on the SML.

The flakes of BSTS are used for a different purpose in the next chapter. It was recently proposed that spin-flip scattering in a TSS can polarize the nuclear spins of the TI. When the current is turned off these nuclear spins are randomized by thermal excitations, at which point they polarize the electrons again. By nature of SML these electrons all flow in the same direction, effectively turning the TI into a battery. A new finding that could perhaps open up a new research area in the field of battery technology. In Chapter 4 I show the results of AC and DC energy retention experiments performed on BSTS flakes. The results show no additional capacitance in the circuit, putting an upper limit to the retention dynamics in this 3D TI. Flakes of two different crystals of $\mathrm{ZrSiS}$ are studied in Chapter 5. This material is known to be a nodal-line semimetal, a peculiar type of Dirac semimetal, and can show an angle-dependent magnetoresistance, known as the butterfly magnetoresistance, that differs greatly from the expected cosine behavior. This chapter studies the differences between the crystals and seeks to find out what Fermi surface morphology lies at the root of the butterfly magnetoresistance. Through the careful analysis of the observed Shubnikov-de Haas oscillations we find that the transport can be carried by trivial or topological modes, depending on the angle of the magnetic field.

Chapter 6 deals with the characterization of the OP of a possible topological superconductor. Superconductor-normal metal side-junctions are made out of flakes exfoliated from a $\mathrm{PdTe}_{2}$ crystal, which is known to be superconducting and is a Dirac semimetal as well. Using tunneling spectroscopy I aim to answer the question whether the superconductivity is also topological and can host MBSs. We will see that the superconductivity has purely conventional $s$-wave pairing, with the exception of a small feature, which could indicate the presence of unconventional helical $p$-wave symmetry of the OP. 


\section{Chapter}

\section{Materials and Methods}

All experiments in this thesis are performed on carefully designed and created nanodevices. The basic element of these devices is an exfoliated flake of topological matter. This chapter gives an overview of the crystals that were used. The research of the past years that has led to these high quality crystals used today is summarized. Additionally, these device design choices are explained in this chapter, in combination with the different ways in which the devices have been made. 
All devices that were created for the experiments shown in this thesis share a common denominator: They have been fashioned out of flakes exfoliated from high quality crystals. Almost all of these crystals were grown by Yingkai Huang from the University of Amsterdam. This chapter lists the design considerations for each of the devices used throughout this thesis, as well as a concise literature overview regarding the relevant materials in the device. Each section deals with one chapter of this thesis, with the exception of chapter 4, which is included in section on the BSTS-devices of chapter 3 since the devices are made from the same material.

\section{$2.1 \quad$ Spin-momentum locking in BSTS}

Chapter 3 describes the experimental verification of the spin-momentum locking in stoichiometric BSTS. For these measurments I have created devices that not only measure the spin-polarization, but also generate and transport the spin information. The complete device thus has all the necessary elements for spintronic applications. Spin-polarization is generated by a TI, transported by a graphene layer, and measured by ferromagnetic electrodes. A full description of the experiments, results, and theoretical understanding is presented in chapter 3 .

\section{Graphene}

Atomically thin layers of carbon, known as graphene, have generated massive scientific, as well as popular, interest since Geim and Novoselov managed to exfoliate these carbon layers from a highly order pyrolytic graphite (HOPG) crystal and create a field effect transistor (FET) structure out of it [69]. Thanks to its small SOC and hyperfine coupling graphene typically has micrometer spin relaxation lengths, making it a perfect material for the transportation of spin information [36, 70, 71]. Theoretically the spin relaxation length in graphene could perhaps even be higher [72]. The choice for graphene in the non-local spin-polarization experiments of chapter 3 is therefore a rather straightforward one. This still leaves the choice of the method of obtaining graphene, as scientists have found a myriad of ways to create graphene [73]. The most notable ones are mechanical exfoliation, like Geim and Novoselov did, and chemical vapor deposition (CVD). Using exfoliation one obtains micrometer sized flakes of graphene that can be deposited on a substrate. The obtained graphene is small, but typically of very high quality and the spin-relaxation length is on the order of a few 
micrometer [70, 74, 75]. Using CVD, graphene layers can be grown in millimeter size on a metallic foil. The process is easily scalable to industrial size wafers and CVD graphene can be bought off the shelf [76]. Having such large patches of graphene is an obvious advantage over exfoliation, but the quality of the graphene is often lower, resulting in a shorter spin relaxation length [77]. The wet transfer process of the graphene to the substrate is more complicated and can negatively influence the quality [78-81], both in terms of spin lifetime as well as substrate coverage, since trapped liquids often rupture the graphene. The latter issue may be resolved by moving to a dry transfer process [82]. In recent years, the spin relaxation performance of CVD graphene has improved and perhaps even surpassed exfoliated graphene $[77,83]$. The performance of CVD graphene as spin transporting material is sufficient for the application in my devices. Having large sheets of graphene instead of micron-sized patches is a large advantage, because TI flakes will be exfoliated and deposited on top of the graphene. Having more graphene enhances the chance of getting a good TI flake on a good graphene sheet. Finally, the choice for CVD graphene over exfoliated graphene, is motivated by the fact that this puts my device one step closer to large-scale applications, as it is a scalable technique.

The sample fabrication starts with the deposition of a CVD grown graphene sheet on an empty substrate. The CVD graphene is supplied on copper foil by Advanced Chemicals Supplier (ACS materials). Both sides of the copper have graphene, but only one layer is needed. One of the layers is removed by RIE with an oxygen plasma, while the other side is protected by a spincoated layer of PMMA. The reactive ion etch is performed with a quite low $\mathrm{RF}$ power of $13 \mathrm{~W}$ for 60 seconds. The oxygen pressure inside the chamber was $7.5 \cdot 10^{-2}$ mbar. Separating the remaining graphene layer from the copper film is done by wet etching the copper using a solution of copper sulfate and hydrochloric acid: $\mathrm{CuSO}_{4} \cdot 5 \mathrm{H}_{2} \mathrm{O}: \mathrm{H}_{2} \mathrm{O}: \mathrm{HCl} \cdot \mathrm{CuSO}_{4}$. $5 \mathrm{H}_{2} \mathrm{O}$ is a blue powder, of which $15.6 \mathrm{~g}$ is used ${ }^{1}$. This is mixed with $44 \mathrm{~mL}$ of $\mathrm{H}_{2} \mathrm{O}$ and $50 \mathrm{~mL}$ of $\mathrm{HCl}$. The PMMA covered graphene on copper floats on this liquid so that the copper is etched away from the bottom until only the graphene and PMMA are left. Using a syringe, and careful pouring, the etchant is taken out of the beaker and replaced with DI water. This process is repeated until the graphene floats in a beaker of nearly pure DI water. The sheet is then scooped out with a $\mathrm{Si} / \mathrm{SiO}_{x}$ substrate. A small droplet of

\footnotetext{
${ }^{1}$ This amount of copper sulfate yields the remaining $6 \mathrm{~mL}$ of water, making ratio of water and hydrochloric acid 1:1
} 

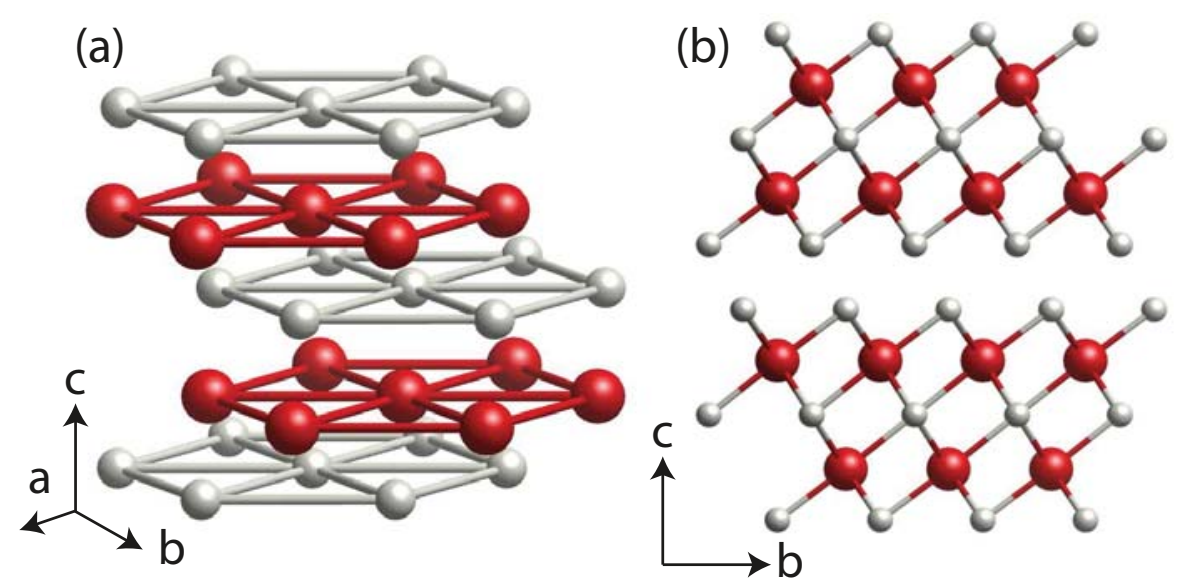

Figure 2.1: Crystal structure of $\mathrm{Bi}_{2} \mathrm{Se}_{3}$ and related compounds. The red spheres represent bismuth and/or antimony atoms. The gray spheres represent tellurium and/or selenium atoms. (a) One quintuple layer. The atoms within the QL are covalently bonded together also between the layers although these bonds are not shown for clarity. (b) Side view (the cb-plane) of the crystal structure. The QL are weakly bonded by van der Waals bonds making the materials easily cleavable along this plane.

isopropanol drives most of the water out from under graphene sheet. Any remaining liquid is absorbed in a tissue gently pressed to the side of the sheet. After blow drying them carefully with nitrogen they are placed on a hot plate of $50{ }^{\circ} \mathrm{C}$. Removing the PMMA protection layer with acetone is the final step of the graphene deposition.

\section{Topological insulators}

The prediction of a topological insulator, albeit not by that name, dates back to 1987 [84], but the field of topological matter has started to grow immensely since the first realization of a 2D TI in $\mathrm{HgTe}$, in 2007 [85]. In the same year, 3D TIs were predicted by Fu and Kane [51] in bismuth compounds that were already studied for their thermoelectric properties. Single crystals of $\mathrm{Bi}_{0.9} \mathrm{Sb}_{0.1}, \mathrm{Bi}_{2} \mathrm{Te}_{3}, \mathrm{Bi}_{2} \mathrm{Se}_{3}$, and $\mathrm{Sb}_{2} \mathrm{Te}_{3}$ were predicted, and found, to be topological insulators with a bulk band gap larger than that of $\mathrm{HgTe}$, which makes the bismuth compounds better suited for applications. Combined with pre-existing knowledge of the bismuth compounds the relatively new field of TI research skyrocketed [86-89]. All of these compounds crystallize in the same rhombohedral structure (space group $\mathrm{R} \overline{3} \mathrm{~m}$ ) as shown in figure 2.1. The unit cell consists of alternating layers of $\mathrm{Bi} / \mathrm{Sb}$ or $\mathrm{Te} / \mathrm{Se}$, 
that come in stacks of five and are therefore called quintuple layers. The bond between two quintuple layers is a weaker van der Waals-bond, which means that the crystal cleaves easily here. Even though these materials do host topological surface states, the bulk states contribute quite heavily to the transport properties, effectively masking the desired TI properties. In the subsequent years ternary and quaternary compounds of $\mathrm{Bi}, \mathrm{Sb}, \mathrm{Te}$, and $\mathrm{Se}$, were created and experimentally verified to have lower, and lower bulk contributions [90-93]. The alloyed $\mathrm{Bi}_{1.5} \mathrm{Sb}_{0.5} \mathrm{Te}_{1.7} \mathrm{Sb}_{1.3}$ exhibits transport almost exclusively dominated by the surface [92], but suffers from a lower mean free path in the TSSs due to disorder in the alloy. The stoichiometric $\mathrm{BiSbTeSe}_{2}$ (BSTS) has slightly more bulk contribution, but grows in a much more ordered way. It also has its Fermi level very close to the DP. Both these things enhance the transport properties of the topological surface states [93, 94]. The advances in TI crystal research were accompanied by parallel efforts to grow the TI compounds in thin layers, instead of single crystals. Many advances have been made in this area of research, moving from the basic two-compound TIs to ternary and quaternary compounds [95-97]. This field is now moving to multilayer stacks, something that cannot be done for single crystals $[98,99]$. At this time the quality of the exfoliated flakes is higher than that of the thin films. For this reason, as well as the aforementioned transport properties, the experiments of chapter 3 and 4 are performed on devices made out of exfoliated flakes of a $\mathrm{BiSbTe}_{2}$ crystal. The crystals are grown by Yingkai Huang at the University of Amsterdam, as described by Li et al. [198].

Depositing the flakes on top of the graphene seems like a challenging task. Usually, not every part of the graphene sheet has survived the deposition process and the BSTS flakes should be deposited on top of the good parts of the graphene, preferably without damaging it. Here we play the numbers game. We deposit a lot of flakes by mechanical exfoliation with residue-free cleanroom foil. Then we search for good flakes on good graphene, neglecting the rest. Typically, three or four of these sites can be identified using an optical microscope. The BSTS flakes should be protected from the etching process that shapes the graphene into strips. The negative e-beam resist AR-N 7520.11 is spun onto the substrate and baked for one minute at $85{ }^{\circ} \mathrm{C}$. This resist requires only a small dose of electrons to write a pattern, $30 \mu \mathrm{C} \mathrm{cm}{ }^{-2}$ is enough. In contrast to the usual positive resists, only the part of the resist that has been exposed remains. The substrate with the graphene and the now protected BSTS flakes is loaded into the 

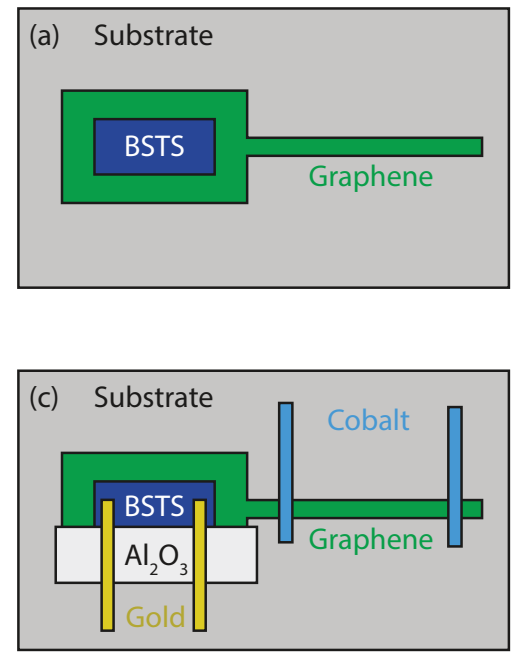

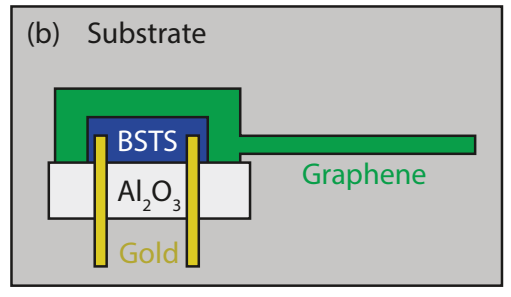

(d)

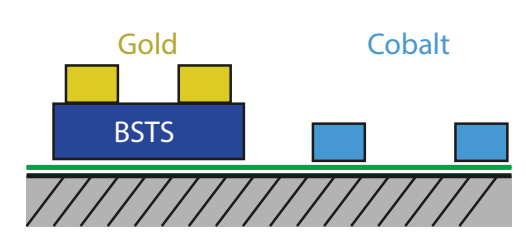

Figure 2.2: Schematic illustration of the fabrication process of the spin-momentum locking devices. (a) Top view. A BSTS flake is deposited on the graphene layer. The graphene has been patterned by EBL and etched in an oxygen plasma. (b) Top view. An $\mathrm{Al}_{2} \mathrm{O}_{3}$ layer is added by PLD to prevent a short from the gold to the graphene. Gold is sputtered over the oxide layer. (c) Top view. In the final step palladium capped cobalt electrodes are evaporated on the thin graphene strip. (d) Side view of the completed device. The normal metal electrodes, indicated as gold in the figure, only touch the top surface of the BSTS. The oxide layer is on the backside and has not been drawn.

RIE, where it is exposed to an oxygen plasma for 30 seconds. We are left with a square of graphene underneath the BSTS and a $400 \mathrm{~nm}$ wide strip that extends for $10 \mu \mathrm{m}$ from the square, as indicated in figure 2.2(a).

Simply growing gold contacts on top of the flake creates a short from the gold to the graphene underneath the flake. By depositing an insulating layer that covers the graphene fully and the flake partially, the short through the graphene is prevented. Using standard electron beam lithography (EBL) with an acceleration voltage of $20 \mathrm{kV}$ and a dose of $250 \mu \mathrm{C} / \mathrm{cm}^{2}$ a square is written in $200 \mathrm{~nm}$ thick PMMA A4 resist and filled with $40 \mathrm{~nm}$ of $\mathrm{Al}_{2} \mathrm{O}_{3}$ by puled laser deposition (PLD) at room temperature in an oxygen background. The PLD step is performed using a laser fluence of $1.5 \mathrm{~J} / \mathrm{cm}^{2}$. The oxide layer is not crystalline, but still does its job of separating the metal contacts from the graphene.

The third EBL step is rather straightforward and, using the standard EBL procedure, makes the pattern for the normal metal contacts that go over the oxide layer and on top of the BSTS flake. The normal metal con- 
tacts themselves are deposited by sputtering and can be gold, palladium, or aluminium. The thickness is typically $80 \mathrm{~nm}$ so that the contact can reach the top of the flake without breaking horizontally. Gold is a favorable metal, because it does not oxidize, but it can be difficult to lift-off. The palladium and aluminium layers suffer far less from this issue. Since aluminium does oxidize, it is capped with $2 \mathrm{~nm}$ of palladium to protect it. A schematic illustration of the device so far is shown in figure 2.2(b).

Now that the device can generate spin in the BSTS and transport it by the graphene, only the read-out part is left. Tunneling into a ferromagnet is a process that depends on the relative orientation of the spin and the magnetization of the ferromagnet. Inspired by Vaklinova et al. no dedicated tunnel barrier is made between the graphene and the ferromagnetic layer, as the interface between the two is enough of a barrier already [101]. The pattern for the ferromagnetic contacts is made using the standard EBL process. Each contact is $500 \mathrm{~nm}$ wide and lies perpendicular to the $400 \mathrm{~nm}$ wide graphene strip. The contacts are made of $40 \mathrm{~nm}$ thick cobalt, which is deposited by e-beam evaporation in a background pressure in the low $10^{-7}$ mbar range. To prevent oxidation of the contacts, they are capped in situ with $10 \mathrm{~nm}$ of palladium. Figure 2.2(c) shows a schematic of the final device viewed from the top and $2.2(\mathrm{~d})$ shows the side view of the devices. 


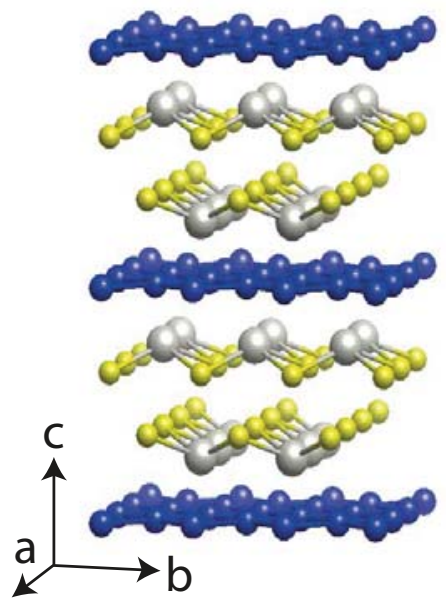

Figure 2.3: Crystal structure is ZrSiS, where the dark blue spheres are the silicon atoms, the gray spheres represent the zirconium atoms, and the small yellow spheres represent the sulfur atoms. The sulfur atoms form two planes that are loosely bound to each other making the crystal easily cleavable along the ab-plane.

\section{$2.2 \quad$ ZrSiS Crystals}

$\mathrm{ZrSiS}$ crystallizes in the tetragonal $\mathrm{PbFCl}$ structure (space group $\mathrm{P} 4 \mathrm{nmm}$ ) and has two square nets of sulfur atoms that face each other in the ab-plane of the unit cell [102], as shown in figure 2.3. These two planes are loosely bound to each other making the crystal easy to cleave along this axis, which is ideal for the mechanical exfoliation of thin flakes. ZrSiS was synthesized in the early 1960s by Dutch and German scientists [103-105], but it was not until 2015 that ZrSiS was theoretically proposed as a topological material [106]. The prediction of ZrSiS having states with linear dispersion that cross the Fermi level in any direction starting from the $\Gamma$-point of the Brillouin Zone (BZ) was experimentally confirmed by angle resolved photo-electron spectroscopy (ARPES) [107]. Nowadays, ZrSiS is recognized as a specific kind of topological material called a nodal line semimetal, where there are not one or a few Dirac points, but a loop of band crossings in the BZ [25]. The enormous magnetoresistance of $\mathrm{ZrSiS}$ and the fact that it is a topological material made of earth-abundant and non-toxic elements have attracted attention from the scientific community $[108,109]$.

The fabrication of the ZrSiS devices on which the experiments in chapter 5 have been performed has less steps compared to the complex BSTS devices, but can be challenging nonetheless. After creating an e-beam lithography or photolithography pattern, 60 to $80 \mathrm{~nm}$ thick gold contacts with a 

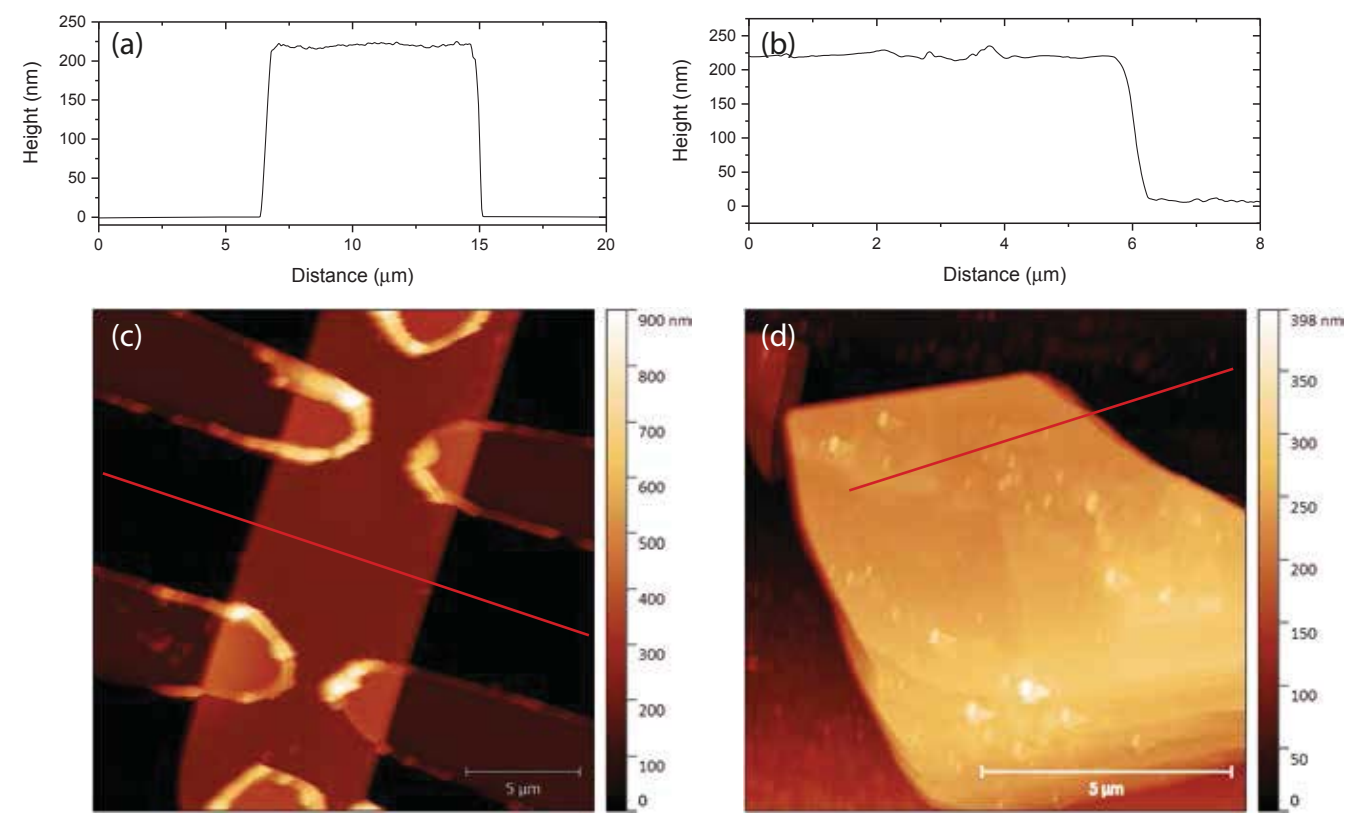

Figure 2.4: AFM scans of $\mathrm{ZrSiS}$ flakes and their corresponding height profiles. (a) and (b) Extracted height profile of a ZrSiS flake of a crystal made by Y. Huang and L. Schoop respectively. (c) and (d) AFM image of a ZrSiS flake of a crystal made by Y. Huang and L. Schoop respectively. The red line indicates the where height traces of (a) and (b) are taken.

$2 \mathrm{~nm}$ titanium sublayer were sputter deposited onto flakes of $\mathrm{ZrSiS}$. These flakes were exfoliated from two different ZrSiS crystals to compare the properties of the two crystals. Two of the flakes are shown in figure 2.4. One of the two crystals was made by Yingkai Huang at the University of Amsterdam, and the other was made by Leslie Schoop from the Max Planck Institute Stuttgart. The ZrSiS crystals made by Schoop gained scientific interest, because they exhibit an angle-dependent magnetoresistance that resembles a butterfly, and is therefore named butterfly magnetoresistance [110]. The origin of the butterfly MR is studied in chapter 5 where I compare and contrast my own experimental findings with what is known about $\mathrm{ZrSiS}$ in literature. Without going into too much detail here, it is clear that the extraordinary electronic properties of $\mathrm{ZrSiS}$ critically depend on the geometry of the BZ. The shape of the ZrSiS Fermi surface in turn depends heavily on the Fermi level. More than it would in a common material. Figures 2.5(b) and (e) show two calculations of the 3D BZ of ZrSiS by different authors $[110,111]$. Even at first glance the similarities and differences between the two are clear. The tubular hole-pockets in the $\Gamma X$-direction are disconnected at $k_{z}=0$ in figure $2.5(\mathrm{c})$ whereas they are fully contected in 

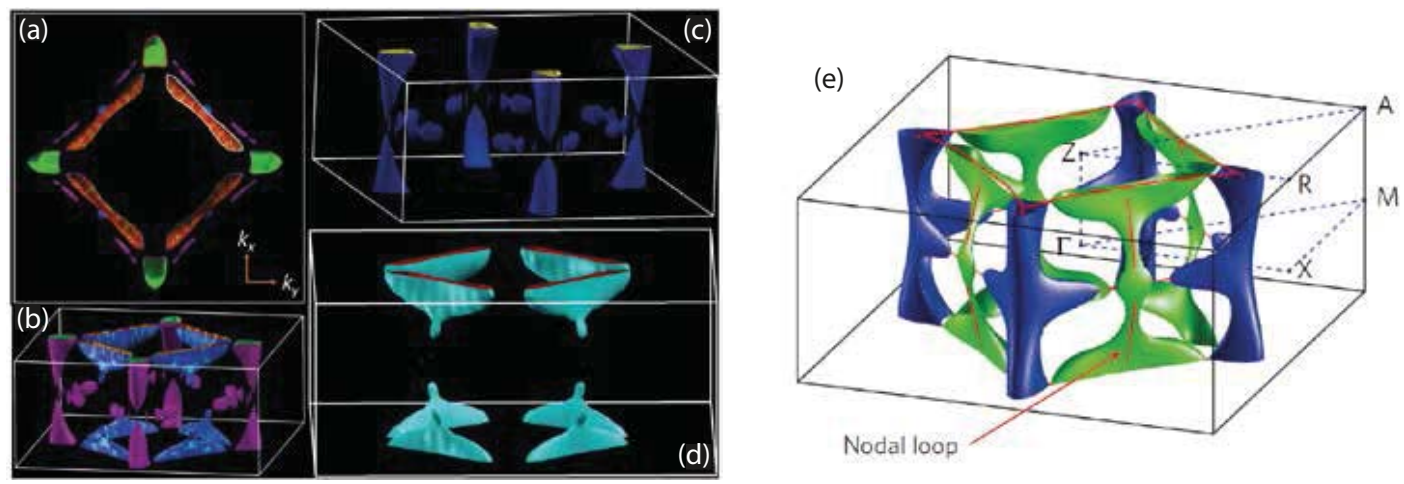

Figure 2.5: Two ZrSiS Brillouin zones. (a)-(d) are calculated by Ali et al. [110]. (a) displays the $k_{x}-k_{y}$ plane of the BZ. (b) shows the full 3D BZ where the hole pockets are colored purple and the electron pockets are blue. (c) and (d) each detail one of the two main features present in (b). (e) shows the BZ calculated by Pezzini et al. that looks qualitatively different [111]. All features are connected through the entire BZ, whereas some are separated in panel (b).

figure 2.5(e) (blue tubes). The small pockets in the $k_{x}-k_{y}$ plane can either be separate (left figure) or connected (right figure) to the tubular hole-pocket. The same reasoning holds true for the electron pocked known as the 'diamond' shape, shown in figure 2.5(d) and in green in figure 2.5(e). All in all, these figures teach us that minor changes can have major consequences; A butterfly effect itself. Figures 2.5(a), (c), and (d) display the $k_{x}-k_{y}$ plane of the BZ, the tubular feature, and the diamond feature respectively. 


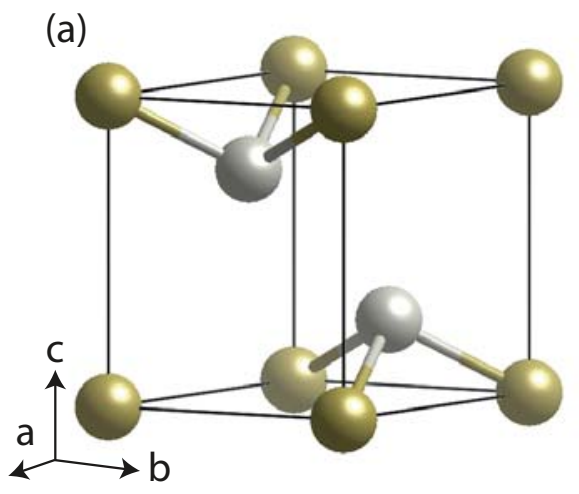

(b)
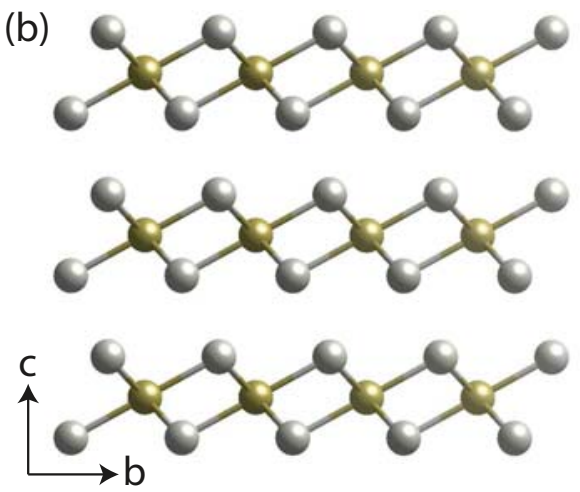

Figure 2.6: Crystal structure of $\mathrm{PdTe}_{2}$, where the gold (gray) colored atoms represent palladium (tellurium). Arrows in the bottom left corner indicate the crystal directions. (a) The unit cell of $\mathrm{PdTe}_{2}$. (b) Cut view of a $\mathrm{PdTe}_{2}$ crystal showing the cb-plane. The material is layered and the natural cleavage plane between the tellurium atoms can clearly be seen.

\section{$2.3 \quad \mathrm{PdTe}_{2}$ superconducting junctions}

$\mathrm{PdTe}_{2}$ is a transition metal dichalcogenide that crystallizes in the $\mathrm{CdI}_{2}$ structure (space group P $\overline{3} \mathrm{~m} 1$ ) as shown in figure 2.6. The synthesis of this crystal dates back to the fifties $[112,113]$ and it was, until recently, mostly known for being a superconductor [114]. In the past few years, physicists have discovered that $\mathrm{PdTe}_{2}$ is a Dirac semimetal as well [115, 117, 204], which raises the question whether or not $\mathrm{PdTe}_{2}$ is a coveted topological superconductor. Recent reports hint towards conventional, type-I superconductivity, but report multiple $\mathrm{T}_{c} \mathrm{~s}$ and leave room for the presence of unconventional superconductivity $[119,120,201]$. The investigation of the symmetry of the order parameter in $\mathrm{PdTe}_{2}$ is presented in chapter 6 . The devices used in this investigation are made from exfoliated flakes of a single crystal of $\mathrm{PdTe}_{2}$ grown by Yingkai Huang from the University of Amsterdam using a modified Bridgeman method, first used by Lyons et al. in 1976 for the synthesis of single crystalline $\mathrm{PdTe}_{2}[121,201]$.

The experiments presented in this chapter have been performed on numerous different devices that share the same feature at the heart of the design: a barrier. The interface between a superconductor and a normal metal, the junction, can teach us about the properties of the superconductor. The quality of this barrier, however, plays a crucial and sometimes underestimated role in the experiments performed on the devices. This section lists the different fabrication processes that have resulted in a wide 

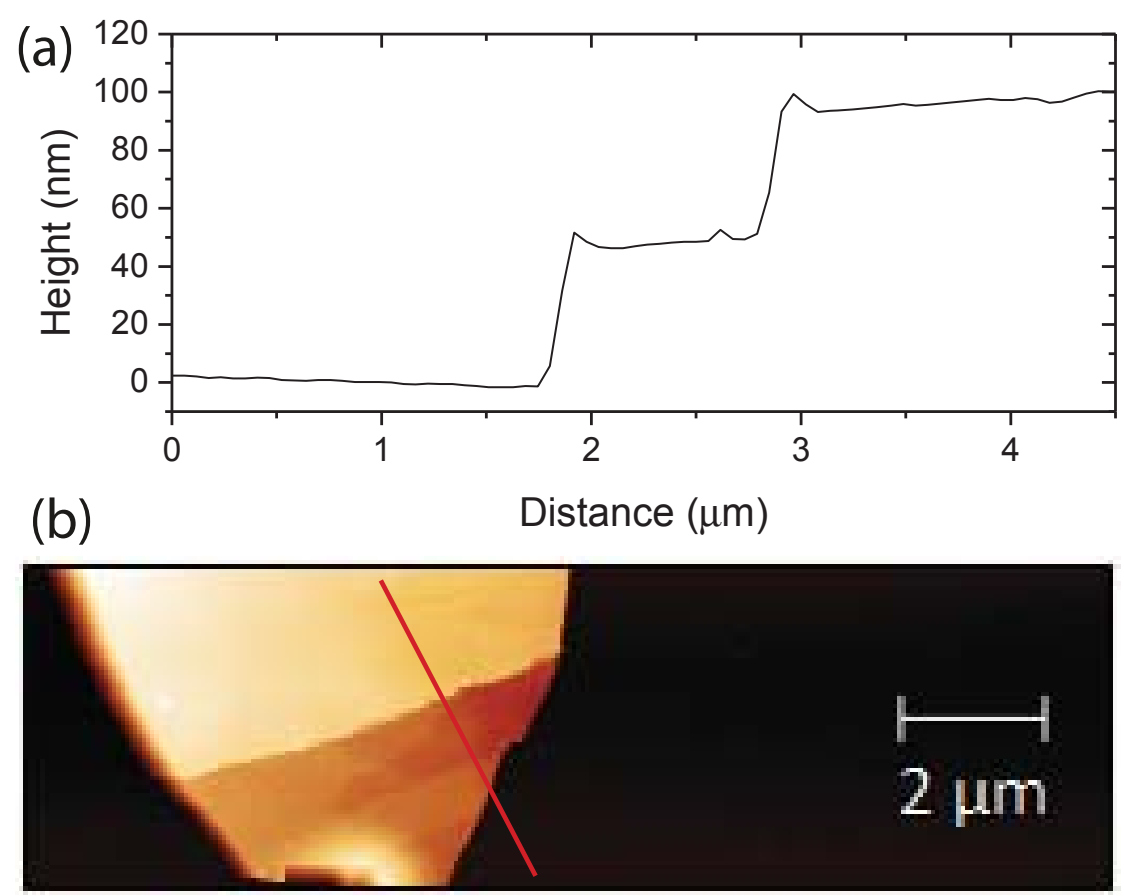

Figure 2.7: AFM scan of an exfoliated $\mathrm{PdTe}_{2}$ flake. (a) Height profile of the flake. (b) AFM image of the $\mathrm{PdTe}_{2}$ flake. The red line indicates where the height profile was taken.

range of devices. All further description of the experiment is presented in detail in chapter 6 .

Since the experiments are aimed at finding the in-plane properties of the order parameter (OP) of $\mathrm{PdTe}_{2}$ the junctions should be made in the ab-direction. $\mathrm{PdTe}_{2}$ is a layered material, which cleaves easily along the abaxis. Thanks to this property we can be sure that the exfoliated flakes have their c-axis pointing along the normal of the substrate. After standard EBL patterning, the first step in fabricating the side-junctions is thus to etch the flake as vertically as possible. The argon milling etch rate with a $25 \mathrm{~mA}$ ion beam and an acceleration voltage of $70 \mathrm{~V}$, is about $10 \mathrm{~nm} / \mathrm{min}$ as verified by AFM. All $\mathrm{PdTe}_{2}$ flakes are scanned by AFM to determine their height and thus the time required to etch through them. An example of such a scan is shown in figure 2.7. After argon milling the sample is transferred to whichever machine is required for the barrier fabrication without removing the resist. This process is called self-alignment, because the next layer fits perfectly to the etched part. The next step, the actual barrier fabrication, is done in four different ways.

The first, and most straightforward way, is to not include an insulating 
layer, thus creating an SN junction. The sample is transferred to a sputter machine and $60 \mathrm{~nm}$ of gold is sputter deposited without cleaning the interface. The edge of the $\mathrm{PdTe}_{2}$ is quite disordered thanks to the argon milling. Even so, the resistance of these kind of devices is low, on the order of tens of Ohms for a junction area of roughly $0.05 \mu \mathrm{m}^{2}$.

The previous process is not very controlled as it relies on side effects of the etching and possible oxidation in the atmosphere. A second way of creating junctions is to first clean the sample inside the sputter chamber, using a low $\mathrm{RF}$ power of $50 \mathrm{~W}$. Then the sample is transferred to the load lock where it is kept in an oxygen environment with a pressure of 10 mbar for one hour. The tellurium of the $\mathrm{PdTe}_{2}$, which is laid bare by the argon milling, will oxidize and form a barrier at the edge. The sample is then transported back to the main chamber where a layer of palladium is grown as the normal metal side of this SIN junction. The resistance of these devices lies around $200 \Omega$. In a different batch this process has been slightly altered. After the cleaning of the sample in the sputter chamber, a $1 \mathrm{~nm}$ layer of aluminum was deposited under an angle of 45 degrees to get an optimal coverage. This layer was then oxidized in the load lock for one hour in an oxygen environment with a pressure of 10 mbar. The palladium normal contact was grown directly afterwards without breaking the vacuum. It is important to not make the aluminum layer too think, as it will not oxidize all the way through. This leaves a conventional superconductor on one side of the interface, thus nullifying the investigation of the $\mathrm{OP}$ of $\mathrm{PdTe}_{2}$. One would only measure aluminum. Although this process seems better on paper, it produced devices with similar resistances: on the order of $200 \Omega$. A future study could investigate multiple aluminum deposition and oxidation steps, to increase the thickness of the $\mathrm{AlO}_{x}$ barrier.

The fourth and final barrier was made using ALD. This very controlled way of growing $\mathrm{Al}_{2} \mathrm{O}_{3}$ produces nicely closed barriers. It was, unfortunately, not possible to transfer the sample from the argon etcher to the ALD system without breaking the vacuum. The ALD grown layer was thus grown on an exposed surface. The same goes for the gold electrode on the other side of the oxide, since the sample was again exposed to air during the transfer to the sputtering machine. The ALD grown oxide layer was $1.2 \mathrm{~nm}$ thick. The resistance of these devices at room temperature was around $2 \mathrm{k} \Omega$, as anticipated. Upon cooldown, unfortunately, most devices increased in resistance to the $\mathrm{M} \Omega$ range. One device remained around $2 \mathrm{k} \Omega$, making it the prime candidate to study the nature of the $\mathrm{OP}$ of $\mathrm{PdTe}_{2}$. 

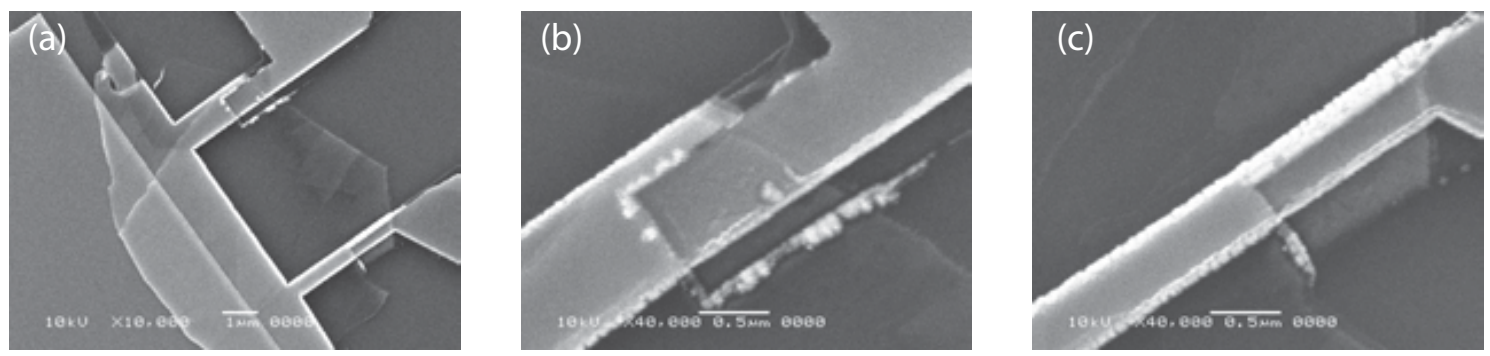

Figure 2.8: SEM images of the $\mathrm{PdTe}_{2}$ junction made by controlled oxidation of the flake. The magnification and scale bar are indicated at the bottom of each panel. (a) Overview of the device. The bottom-left corner shows niobium. The flake is in the middle, and the gold in the top-right corner. (b) and (c) each show a zoomed in picture of one of the junctions. The bottom-left part is $\mathrm{PdTe}_{2}$ and the top-right is gold.

As the final step of the fabrication the surplus of $\mathrm{PdTe}_{2}$ surrounding the junction is removed by argon milling. From the first etching process the time it takes to fully etch through the flake is determined, which can be used for this second etching process. The result of the sample fabrication is shown by the SEM images of figure 2.8. 


\section{Chapter}

\section{Spin-momentum locking in the gate tunable topological insulator BiSbTeSe 2 in non-local transport measurements}

The helical spin-momentum locking of an electron in a topological surface state is a feature excellently suited for the use in spintronic applications. We have fabricated devices that allow us to generate, transport, and detect the spin-polarization coming from an electronic current in the topological surface state of BiSbTeSe 2 ; a topological insulator reported to have a negligible bulk contribution to its conduction. This chapter describes the successful creation of such a device, as well as a study of the generated spin-polarized current as the BiSbTeSe $e_{2}$ surface state is gated through its Dirac point. A non-local voltage difference across separated ferromagnetic leads is observed, larger than previously reported in literature. The spinpolarization has a maximum when the Fermi level crosses the Dirac point. 


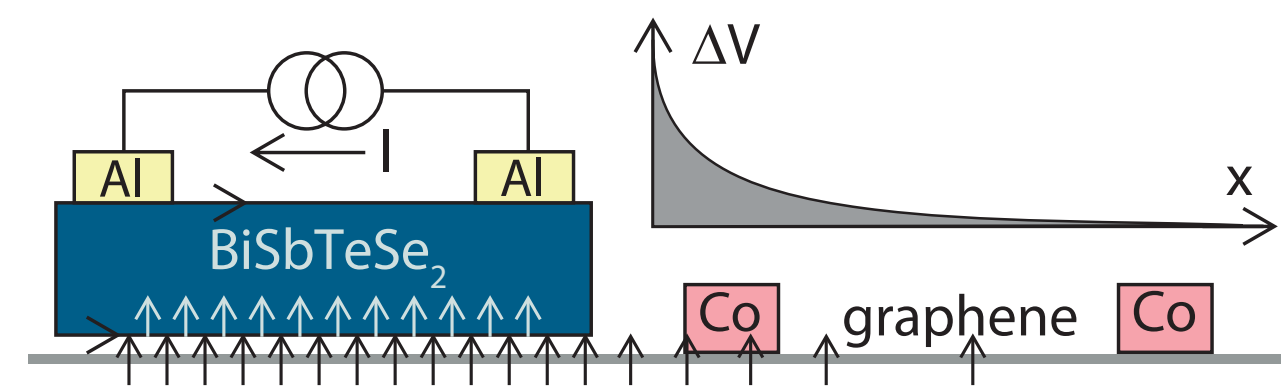

Figure 3.1: Schematic drawing of our device. The $150 \mathrm{~nm}$ thick BSTS flake rests on top of a CVD grown sheet of graphene, patterned into a strip. The BSTS is fitted with two Al contacts to drive a current, which is spin-polarized due to the SML. This spin-polarization, indicated by the arrows, diffuses through the graphene to the cobalt leads, where a voltage is picked up. The top right graph indicates the decay of the spin-polarization in the graphene channel.

\subsection{Introduction}

In the past decades there has been an ever-increasing interest in spintronic devices, which promise to be faster and more energy efficient than their charge based counterparts. On the road towards spintronic devices there is the challenge of generating, storing, transporting, and reading spininformation in nanoscopic devices. Three-dimensional topological insulators (TIs) are a recently discovered class of quantum matter, touted as a way of solving these problems. TIs have an insulating bulk, but are host to spinmomentum locked states on their surface [32-34, 50, 51]. This remarkable feature, where the spin and momentum of an electron in the topological surface state (TSS) are coupled to each other, has been demonstrated experimentally by spin and angle resolved photon emission spectroscopy (spin ARPES) [122-125], as well as a number of different spin-transport experiments $[101,126-137]$. Perhaps the most notable spin-related property in TIs is their record-breaking spin-transfer torque, which could prove very useful for spintronic applications such as memory cells and computational devices $[67,68,138]$. In many of the spin-transport experiments it has been shown that the observed spin-polarization depends linearly on the applied bias current through the TSS. It is therefore an essential property of this effect that the observed spin-polarization reverses as the applied bias current reverses, as there has been an observation of a similar effect, which does not reverse as the bias current reverses [134, 159].

Experimental investigations of the spin-momentum locking (SML) in TIs 
Chapter 3. Spin-momentum locking in the gate tunable topological insulator $\mathrm{BiSbTeSe}_{2}$ in non-local transport measurements

have been primarily focused on devices measuring the spin-polarization in a local configuration, i.e. the magnetic pick-up contacts lay in between the source leads. In a recent work by de Vries et al. [134] it was shown that there may be various influences on the measurements performed on such devices. Vaklinova et al. have found a solution to this problem in using a graphene sublayer as a way of transporting the spin-polarization, thereby measuring in a non-local configuration [101]. In their work it is suggested to improve the device by moving from a TI with considerable bulk conduction to a TI that has dominant and gate-tunable surface states. Here, we have realized this non-local and gate tunable configuration. First, we have used flakes exfoliated from a BiSbTeSe 2 (BSTS) single crystal, reported to have a minute bulk conduction contribution once the flakes are thin enough $(<$ $100 \mathrm{~nm}$ ) [140]. Additionally, we have made this device backgate-tunable. Not only does this allow us to remove small bulk contributions from the signal, but it enables the tuning of the Fermi level through the Dirac point (DP), moving from electron ( $\mathrm{n}$ ) to hole ( $\mathrm{p}$ ) type carriers. The continuous tuning of the Fermi level provides advantages over a similar study by Yang et al. of distinct n- and p-typed doped samples [135]. In this chapter we first report on spin potentiometric measurements performed on a BSTS flake in a non-local configuration as shown in figure 3.1 to show that SML can be observed in the device. The second section of this article describes gating experiments performed on a different BSTS/graphene device.

\subsection{Proof of principle}

We have fabricated devices out of a $150 \mathrm{~nm}$ thick BSTS flake mechanically exfoliated on top of a CVD grown sheet of graphene. Chapter 2 gives a detailed description of the choices made in the fabrication of the devices. For clarity, a brief summary is also included here. The graphene layer was patterned into a strip by e-beam lithography and etching with an oxygen plasma. Using pulsed laser deposition, a layer of $\mathrm{Al}_{2} \mathrm{O}_{3}$ is grown, that serves the purpose of electrically isolating the top contacts from the graphene sheet. Over the oxide layer two aluminum contacts with a $2 \mathrm{~nm}$ palladium capping are sputter deposited, so that contact is made only to the top surface of the BSTS. Finally, cobalt contacts are patterned and grown by e-beam lithography and e-beam evaporation. Since cobalt, like aluminum, oxidizes quite quickly these contacts are capped with a $10 \mathrm{~nm}$ palladium layer. Because of the complicated fabrication the device yield is low. Below, we describe 
two functional devices.

We cool a device down to $4.2 \mathrm{~K}$ and send a current through the BSTS flake. This means that there is an imbalance in the momenta of the electrons. There are more electrons moving in one direction, than in the other. Because the electron spin is locked to the momentum a spin-polarization is generated at both TSSs by the current applied in the BSTS and the spinpolarization at the bottom TSS diffuses to the graphene. In the graphene layer the spin-polarization decays with distance from the TI flake, since the electrons are subject to spin-flip scattering. The spin relaxation length in CVD graphene is on the order of several micrometer. Because of the decay, an observable difference in voltage $(\Delta \mathrm{V})$ is created between the two ferromagnetic (FM) cobalt leads. Figure 3.1 shows a schematic illustration of this in the top right corner. The arrows on the graphene indicate the spinpolarization. We have measured the voltage between the FM leads as a function of the magnetic field applied parallelly to the FM leads using lockin techniques. The spin-polarization is generated by applying a bias current through the BSTS together with an AC excitation. We have repeated this measurement for bias currents between $-8 \mu \mathrm{A}$ and $+8 \mu \mathrm{A}$. When the magnetization of the FM leads switches direction it becomes parallel (antiparallel) to the spin-polarization in the graphene underneath yielding a high (low) voltage difference, observable as a step in the measured voltage. Figure $3.2(\mathrm{a})$ and (b) show the results of two of these non-local spin transport measurements performed on our BSTS/graphene device. In figure 3.2(a) a DC bias current of $5 \mu \mathrm{A}$ was applied on top of a $2 \mu \mathrm{A} \mathrm{AC}$ current, whereas the opposite DC current, $-5 \mu \mathrm{A}$, was applied for figure 3.2(b). Upon sweeping a magnetic field, parallel to the FM leads down (black) and up (red), the non-local differential resistance changed in a step-like fashion around the coercive field of the FM leads. A constant offset of about $400 \Omega$ was subtracted from the measured differential resistance, $\mathrm{R}_{N L}$, defined as $\frac{d(\Delta V)}{d I}$. The finite slope of the step is caused by the switching of individual ferromagnetic domains in the cobalt leads, effectively smearing out the transition. The step in the non-local resistance indicates the presence of a spin-polarization underneath the FM leads. When the bias current is inverted the observed hysteresis loop also inverts, which shows that the spin-polarization in the graphene channel is indeed generated by the BSTS flake.

In figure 3.2(c) this is investigated further. This graph shows the step in non-local voltage observed in magnetic field sweeps for bias currents from -8 
Chapter 3. Spin-momentum locking in the gate tunable topological insulator BiSbTeSe $e_{2}$ in non-local transport measurements
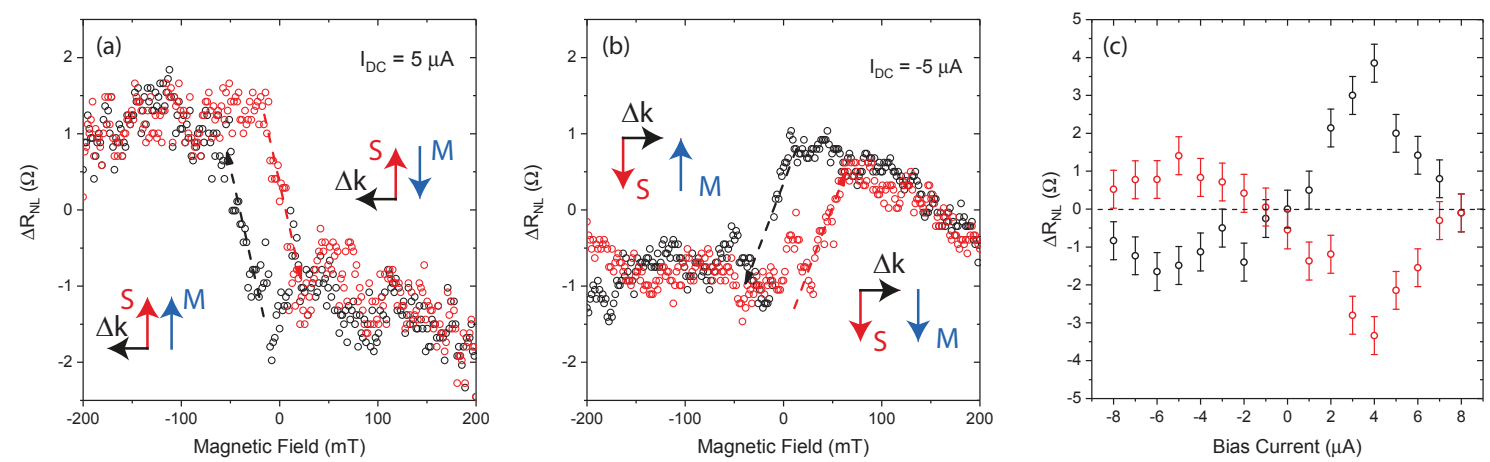

Figure 3.2: Non-local resistance measurements between two FM leads vs magnetic field for bias currents of $+5 \mu \mathrm{A}$ (a) and $-5 \mu \mathrm{A}$ (b) and an $\mathrm{AC}$ excitation of $2 \mu \mathrm{A}$. The black and red curves represent the down- and upsweep respectively. The arrows indicate the direction of the shift in momentum (black), spin-polarization (red, defined as the spin expectation value along the $\mathrm{k}_{x}$ direction) and magnetization of the FM leads (blue). A parallel spin-polarization and magnetization lead to a high non-local resistance. (c) Step in non-local resistance vs applied bias current. Black and red represent the down- and upsweeps respectively. The errorbars reflect the noise in each measurement. It is clear that the behavior is opposite for positive and negative bias currents showing that the observed spin-signal stems from the TSS.

$\mu \mathrm{A}$ to $+8 \mu \mathrm{A}$. Black and red datapoints represent the down- and upsweeps respectively. The errorbars reflect the fluctuations in the sweeps, that can be seen in figure 3.2(a) and (b), for example. For negative bias currents the black (red) datapoints have negative (positive) values, whereas the opposite is true for positive bias currents. The inversion of the hysteresis loop in bias current is thus a robust feature in our device, proving that the SML in the BSTS SS generates the spin-polarization that we observe. Additionally, we found that the spin signal, $\Delta \mathrm{V}$, depends linearly on the applied current for small currents $(<4 \mu \mathrm{A})$ as is shown in figure 3.2(c), showing that the $\mathrm{SML}$ in the TSS is the source of this effect. For larger currents $\Delta \mathrm{V}$ decreases, which we attribute to increased scattering due to Joule heating, in a similar vein as Vaklinova et al. [101].

Besides the loop inversion, there is another asymmetry present in figure $3.2(\mathrm{c})$ shows $\Delta \mathrm{R}_{N L}$. There is a noticeable difference between the $R_{N L}$ for negative bias currents and positive bias currents. This can tentatively be understood as a Schottky barrier between the metal contact and the TI. The presence of a Schottky diode can create an asymmetry between a positive and negative applied voltage, which is reflected in the strength of 
the measured signal. Theoretically, one expects the non-local resistance to increase linearly with the applied bias current [141, 142]. Overall, it is clear that the design works as intended and we can show the presence of SML in a transport measurement.

\subsection{Tuning the Fermi energy}

By applying a voltage difference between the flake and the bottom of the substrate we can tune the number of charge carriers present in the BSTS, which is equivalent to moving the Fermi level in energy. After all fabrication steps, the BSTS flakes typically have their Fermi level slightly above the Dirac point, but not in the bulk conduction band. The charge transport is carried by electrons in this regime. When we remove electrons, by applying a negative voltage to the back of the substrate with respect to the current drain on top of the BSTS flake, the Fermi level moves down in energy. Once it crosses the DP the current will be carried by holes instead of electrons [143]. Figure 3.3 shows schematically what this would mean for the generated spin-polarization. Contrary to what one would think at first sight, changing from n- to p-type carriers has no influence on the sign of the spinpolarization. Panel (a) shows the filling of the Dirac cone when a current that is carried by electrons is applied. Some negative $k$ states have moved to the positive $k$ side, which gives a net spin-polarization, as indicated by the red arrows. The dotted line indicates the tilt in the Fermi level, because of the applied current ${ }^{1}$. Figure $3.3(\mathrm{~b})$ shows the same situation, with the exception that the current is carried by holes instead of electrons. Because the applied current is the same, the holes move in the opposite direction as the electrons did. The current creates more holes on the negative $k$ side by moving more electrons into the positive $k$ side. The red arrows again indicate the spin that is associated with the branch of the Dirac cone. The holes that carry the current provide the same spin-polarization as the electrons did [135].

Figure 3.4 shows the main results obtained on a second device, similar to the first, but fitted with a backgate. We have repeated the measurements described in section 3.2 at different gate voltages. In our device the gate voltage is applied over both the graphene layer and the BSTS flake. From

\footnotetext{
${ }^{1}$ This way of drawing the Fermi level is both easy and insightful for the viewer. Please note that a Fermi level is a concept that, by definition, has only one energy. A more physically accurate drawing would show the line straight, but the cone tilted.
} 

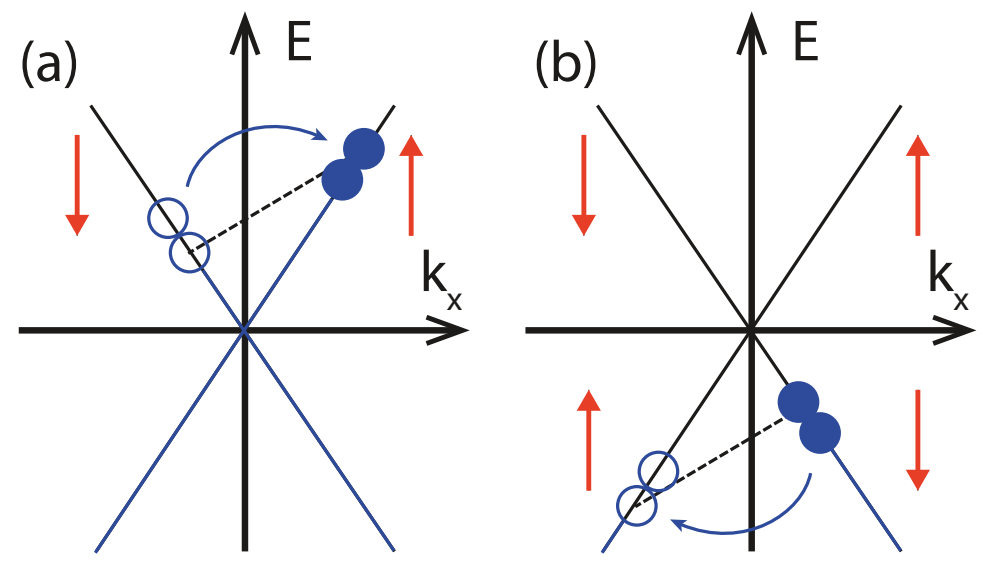

Figure 3.3: Schematic illustration showing the spin-polarization, as the result of an applied current, in a Dirac cone with SML. Filled and open circles represent filled and empty states respectively. The red arrows indicate the spin associated with the branch of the Dirac cone, where an up-arrow means spin pointing in the $k_{x}$ direction. (a) The current is carried by the n-type carriers. More electrons move to the positive $k$ states, which gives a net spin-polarization. (b) The current is carried by the p-type carriers. More holes are present in the negative $k$ side. This is still the same branch as for electrons. We thus obtain the same spin-polarization.

the measured two-probe resistance of the BSTS flake as a function of the gate we determine that the Dirac point lies at $V_{B G}=-65 \mathrm{~V}$, which is shown by the solid blue line in figure 3.4(c). The dashed black line serves as a guide to the eye. The carrier density of the graphene layer is sufficiently low that it cannot screen the electric field, which is the reason why we are still able to observe an effect of the back gate on the top surface of the BSTS. Typical carrier densities for CVD graphene are reported to be smaller than 4 . $10^{12} \mathrm{~cm}^{-2}[78,83,144,145]$. We are therefore able to measure the step in $R_{N L}$ above and below the Dirac point. The results of these measurements are shown in figures 3.4(a) and 3.4(b). The black and red curves in both fig. 3.4(a) and (b) represent the down- and upsweeps of the magnetic field respectively and can be transformed into each by adding a minus sign to $\Delta R_{N L}$ showing that a hysteresis loop is formed for all measurements. Figure 3.4(c) shows the results of magnetic field sweeps at backgate voltages between $0 \mathrm{~V}$ and $-100 \mathrm{~V}$. The bias and excitation currents were kept at $4 \mu \mathrm{A}$ DC plus $1 \mu \mathrm{A} \mathrm{AC}$. Although the large, eye-catching peak is situated at $V_{B G}$ $=-40 \mathrm{~V}$ it is still likely that it relates to the crossing of the DP at $V_{B G}=-65$ $\mathrm{V}$. The normal metal contacts used to source the current through the flake only contact the top surface of the BSTS flake, while the backgate mostly influences the bottom surface. It stands to reason that the voltage required 
to reach the DP in the top surface is higher than the voltage required to reach the DP in the bottom surface. This is visible in the discrepancy between the maximum of the solid line and the open dots in figure 3.4(c). When the Fermi level crosses the DP the non-local voltage difference does not change sign, highlighting the fact that the spin-polarization is unaltered when the system moves from n-type to p-type carriers, as was also suggested in a previous study performed by Yang et al. [135].

Figure 3.4(a) is qualitatively similar to the measurements performed on the first sample, shown in figure 3.2. There is a linear slope around $0 \mu \mathrm{A}$ and the curve bends back towards 0 when the applied current reaches values greater than $5 \mu \mathrm{A}$. It appears, however, that in both the down- and upsweeps an additional effect is present, which provides a constant offset in $\Delta R_{N L}$. The most striking difference between the measurements of the two devices is the size of the step in the $R_{N L}$, which is much larger in figure $3.4(\mathrm{a})$.

\subsection{Discussion}

At a much larger negative gate voltage the graph is different, except for the fact that the same constant offset effect is present in figure 3.4(b) as in 3.4(a). The difference between the two is that instead of the antisymmetric behavior of fig. 3.4(a), fig. 3.4(b) is symmetric in bias current. We believe that at these large voltages the Fermi level has left the Dirac cone of the TSS altogether and we are now looking at the transport properties of the BSTS bulk. Effects such as the direct Rashba-Edelstein effect (DREE) and the (inverse) spin Hall effect (SHE) become relevant in this regime. The DREE could, in principle, yield a spin-polarization like a TSS would, but it is expected to be smaller since there are always two Fermi surfaces with opposite helicity [141]. The SHE is a phenomenon where a spin-polarization is created from a charge current $[60,61]$, just as the measurements in this chapter show. The SHE, however, can be ruled out since the charge current and spin-polarization are necessarily orthogonal to each other and our devices only probe longitudinal effects.

A different explanation for the non-inverting $\Delta R_{N L}$ can be found when we consider imperfections in the device. CVD grown graphene that is transferred to a substrate using a wet transfer method, as used in this chapter, 
Chapter 3. Spin-momentum locking in the gate tunable topological insulator BiSbTeSe $e_{2}$ in non-local transport measurements
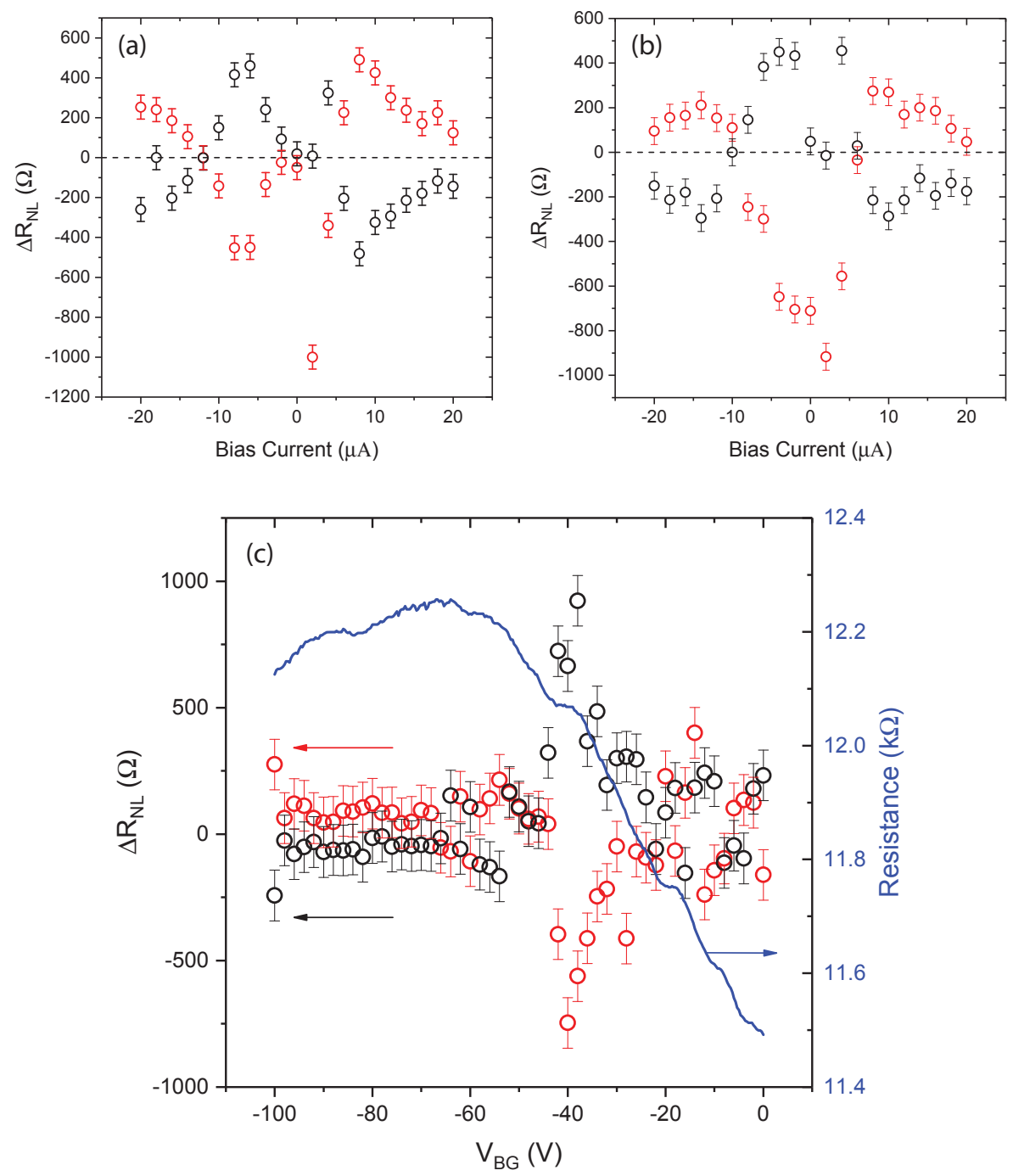

Figure 3.4: (a) and (b) show the steps in $R_{N L}$ extracted from magnetic field sweeps for constant DC bias currents from $-20 \mu \mathrm{A}$ to $+20 \mu \mathrm{A}$. The gate voltage was set to $V_{B G}=-20 \mathrm{~V}$ (a) or $V_{B G}=-80 \mathrm{~V}$ (b), which puts $E_{F}$ above or below the DP respectively. Black and red points represent the up- and downsweeps, showing that a hysteresis loop is obtained for all sweeps. The curve is qualitatively similar to our previously obtained result. (c) Steps in $R_{N L}$ extracted from magnetic field sweeps at backgate voltages ranging from $0 \mathrm{~V}$ to $-100 \mathrm{~V}$. The applied current was 4 $\mu \mathrm{A} \mathrm{DC}$ plus $1 \mu \mathrm{A} \mathrm{AC}$. The solid blue line shows the two-point resistance measured on the top surface of the BSTS as a function of the gate voltage. The vertical dashed line indicates the Dirac point and serves as a guide to the eye. 
has been reported to have even smaller scattering lengths than dry transferred CVD graphene, a material known to have smaller scattering lengths than exfoliated graphene, which can typically have spin relaxation lengths of $2 \mu \mathrm{m}[74,78,146]$. The lowered spin relaxation length in CVD graphene is primarily due to the presence of metallic adatoms such as copper, increasing the SOC [79-81]. During the gating we also heavily influence the relaxation length of the spin-polarization in the graphene layer, as was recently reported [147]. Another device feature to consider is the (tunnel)barrier between the graphene and the cobalt, which is likely to be different from contact to contact. There is no intentional oxide barrier between these layers, but rather a bad, and gate-dependent, contact between the graphene and the cobalt. In section 3.2 it was shown that this is sufficient to observe the spin-polarized current, but once the barriers start to differ from each other they may influence the measurements. Upon changing the magnetic field the tunneling probabilities for the two spin species should exactly invert. It is not unimaginable that for one or more of the FM leads this inversion is not complete. Perhaps the barrier strength is weaker for one of the leads, which limits its ability to distinguish between the spins. Figure 3.5 shows schematic band diagrams of such a FM lead. This difference between the individual cobalt leads is a likely cause of additional steps in non-local voltage, which may not invert with the flipping of the spin-polarization. Furthermore, the electronic band structure of BSTS may in practice be more complex than it is often drawn theoretically. Recent reports on BSTS and a related compound find a large influence of charge puddles on the transport properties $[148,149]$. Fluctuations of the bulk bands of BSTS may very well influence the spin-polarization. The presence of charge puddles is most notable in a gating experiment since a fixed Fermi level may lie outside of the charge puddles thus obscuring any effect related to the puddles.

There is another possible reason, that should not go unmentioned, for having a $\Delta R_{N L}$ that does not invert with the bias current. Backscattering in a TI must always be accompanied by a rotation of the spin, a consequence of SML. The spin in this process is conserved by the nuclear spins. So applying a current through a TI aligns the nuclear spins parallelly, very much like a battery. Tian et al. have recently reported experimental evidence of a non-inverting $\Delta R_{N L}$. They attribute this to this interaction between the electron spins and the nuclear spins [159]. The effect observed by Tian et $a l$., however, is of the order of several Ohms, which is smaller than what is observed here. In principle, our devices should be capable of showing this 
Chapter 3. Spin-momentum locking in the gate tunable topological insulator BiSbTeSe $e_{2}$ in non-local transport measurements
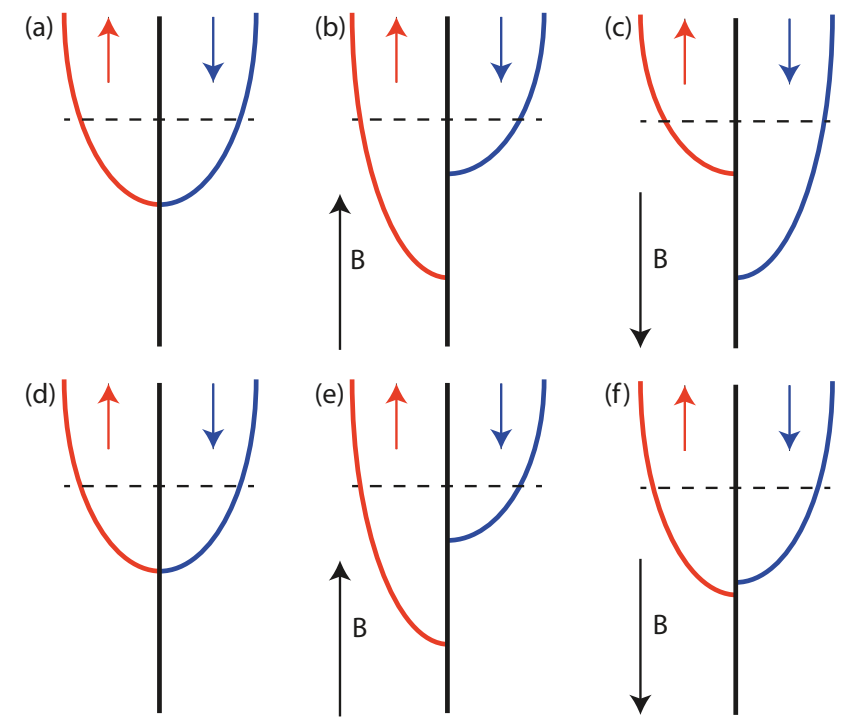

Figure 3.5: Schematic representation of the spin bands (red and blue arrows) in a ferromagnetic contact. The area of the cross-section of the Fermi level (dashed line) and the band is a measure for the amount of spins that tunnel in, and thus the obtained voltage on that lead. (a)-(c) Show the inversion of the magnetization for a perfect contact. The magnetic field is indicated for each illustration. For (a) and $(\mathrm{d})$ there is no field and no internal magnetization. (d)-(f) represent the same thought experiment, but with an imperfect ferromagnetic lead. This situation does not lead to an inversion of $\Delta R$. 
storage of spin information in the nuclear spins. The next chapter (chapter 4) describes the experiments investigating the storage of energy as spin information.

\subsection{Conclusions and outlook}

The steps in the non-local resistance reported in figures 3.2(c) and 3.4 are extracted from magnetic field sweeps such as those reported in figure 3.2(a) and (b). These steps are measured around the coercive field of the cobalt leads, where the effect we are interested in should, and does, occur. We want to stress, however, that not every sweep is successful. Even at field values far from the coercive field, there are occasional steps that in some cases do not step back to the original value upon reversing the magnetic field. Even though the results reported in this work are quite clear, the stability and reproducibility of the devices should be improved upon when one moves to spintronic applications of the spin-momentum locking in TIs. This may be related to the complexities of the fabrication process, or to the effect of the nuclear spins in the TI on the spin-polarization measurements. How the nuclear spins influence the spin-related transport properties of the BSTS will be investigated in the next chapter.

In summary, we have demonstrated that the spin-polarized signal measured in a non-local fashion, is generated by the spin-momentum locking in the TSS of the BSTS flake. BSTS has been shown to be a suitable candidate for usage in spintronic applications, both as a spin generator and spin transporter. Furthermore, we have investigated the behavior of the spin-polarized current when the Fermi level crosses the DP. Although conclusive evidence remains elusive, our results indicate that the efficiency of spintronic devices based on TIs is improved by having the Fermi level close to the DP. 


\section{Chapter}

\section{Upper limit to the energy retention in the 3D topological insulator $\mathrm{BiSbTeSe}_{2}$}

The hyperfine interaction between spin-momentum locked electrons in a TI surface state and the nuclear spins of the lattice atoms has been predicted as a way to enable storing energy as information and releasing it as energy again, effectively making the TI a spin battery. In this chapter I present both $D C$ and $A C$ measurements performed on three different BiSbTeSe $e_{2}$ samples investigating the storage of energy. In the slow $D C$ measurements there is no evidence of retention. The faster AC measurements similarly show that the capacitance associated with the decay of an applied voltage is no larger than what is measured on test resistors, putting an upper limit on the battery performance. Although a solid theoretical framework in three dimensions is required to understand all intricacies of the measurements, the experiments in this chapter put a clear upper limit on the performance of a BiSbTeSe $e_{2}$ spin battery. 


\subsection{Introduction}

In an ideal two-dimensional TI all charge transport occurs at the edges of the material, as was described in section 1.3. These edge states carry the current very efficiently since the spin-momentum locking prevents the carriers from scattering elastically. The transport in these $1 \mathrm{D}$ channels, however, is not resistanceless. Quite a few mechanisms have been suggested to contribute to the resistance of the edge mode. These include scattering on magnetic impurities $^{1}$ [150-152], the influence of the Overhauser field [154], and the interaction between electrons and the nuclear magnetic moment of the lattice atoms, known as hyperfine interaction [155-158]. The Overhauser field is the collective field produced by the aligned nuclear spins in the material, which can be maintained, for example, by the RKKY interaction [157]. This magnetic field tilts the two spin states in the same direction so that the left and right-moving edge state are no longer orthogonal and backscattering is now possible, which leads to resistance. Although the RKKY mechanism only plays a role at low temperature $[157,158]$, an Overhauser field should be considered at all temperatures. Recently, the hyperfine interaction has been suggested to be a key ingredient in the storage of energy in a topological insulator $[159,160]$.

In a TI any applied current is spin-polarized by the SML and the carriers can transfer their spin to the nuclear spins through the hyperfine interaction, aligning the nuclear spins in one direction. When the current is stopped, the nuclear spins relax to a non-polarized state by thermal processes. Upon relaxing, the spin information is transferred back to a charge carrier, that thanks to SML also obtains a certain momentum. These carriers move to one side creating an equilibrium potential across the device, much like a capacitor. When the device is left unconnected this open-circuit voltage should be maintained indefinitely. Attaching a load to the circuit allows one to extract work from the battery. Extracting work from nothing more than information is a revolutionary new method of storage, that has the potential to dramatically change battery technology. Tian et al. [159] have found experimental indications of the storage of information in a TI. Mert Bozkurt et al. have recently written a theoretical analysis of work extraction from a spin battery [160]. This chapter contains the report of experiments performed on three different devices, investigating the storage of and ex-

\footnotetext{
${ }^{1}$ Tanaka et al. have demonstrated that a single magnetic impurity does not change the DC electrical conductance [153], but multiple do
} 
Chapter 4. Upper limit to the energy retention in the 3D topological insulator BiSbTeSe 2

traction of work in a TI.

A spin battery made from a TI flake is, in essence, nothing more than a flake of a TI and two metallic contacts. The flakes were exfoliated from a high quality single-crystalline $\mathrm{BiSbTeSe}_{2}$ crystal and have thicknesses ranging between $80 \mathrm{~nm}$ and $200 \mathrm{~nm}$, which is thick enough to decouple the top and bottom surface. Two types of devices were made from these flakes. The first type (sample 1) has gold contacts that, with the use of ALD grown $\mathrm{Al}_{2} \mathrm{O}_{3}$, are decoupled from the side and bottom and thus only touch the top surface of the flake. The gold contacts are $12 \mu \mathrm{m}$ apart and the flake has a width of $13 \mu \mathrm{m}$. The second set of devices have niobium leads that cross the flake forming wide, but short channels ${ }^{2}$. The channels range in length between $200 \mathrm{~nm}$ and $300 \mathrm{~nm}$ and have a widths of $1.5 \mu \mathrm{m}$ (sample 2), and $7.3 \mu \mathrm{m}$ (sample 3).

\subsection{Measurements}

The devices, shown in figure 4.1(a), are connected to a measurement circuit designed to test the perfomance of the device as a battery. This circuit is shown schematically in figure 4.1(b). Using two switches, all three parts of a battery cycle can be tested. Using a switch to close the circuit, a constant $\mathrm{DC}$ voltage is sourced over a $10 \mathrm{k} \Omega$ resistor in series with the device. The constant current that runs through the BSTS is spin-polarized thanks to the SML and can thus polarize the nuclear spins upon scattering. Opening this switch again effectively creates an infinite load on the system. During this holding step an open-circuit voltage should be generated by the device. Closing a second switch connects the device to a $100 \mathrm{k} \Omega$ resistor to discharge the spin battery. The experiment cycles through the three steps in the following order: charging, holding, discharging, holding. Each step is maintained for 10 seconds. The results of the experiment on sample 1 are shown in figure $4.1(\mathrm{c})$ as well as a zoomed in version in (d). These measurements have also been performed on several channels of samples 2 and 3 and have yielded the same results. During the holding step of the cycle there will always be a finite offset voltage. To distinguish an offset in the voltmeter from an offset created by the holding voltage, the experiment is performed using a positive and a negative charging current. The holding voltage should depend on the charging current, which seperates it from any systematic offset in the measurement equipment. The black line

\footnotetext{
${ }^{2}$ These devices were made by Bob de Ronde at the University of Twente.
} 


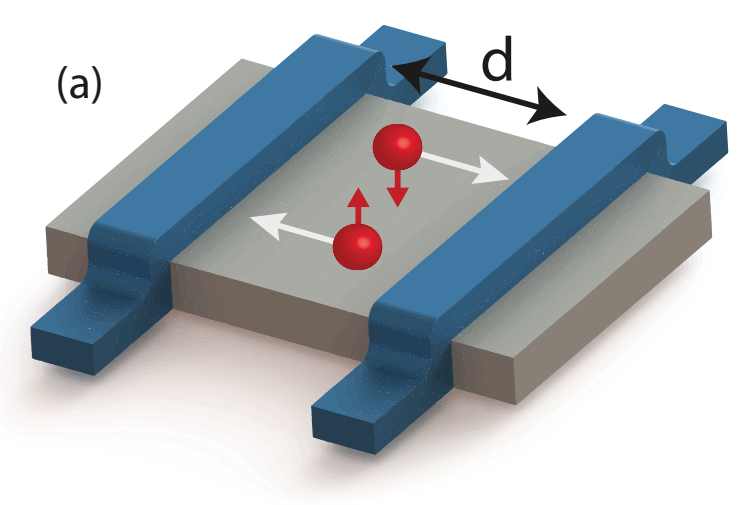

(b)

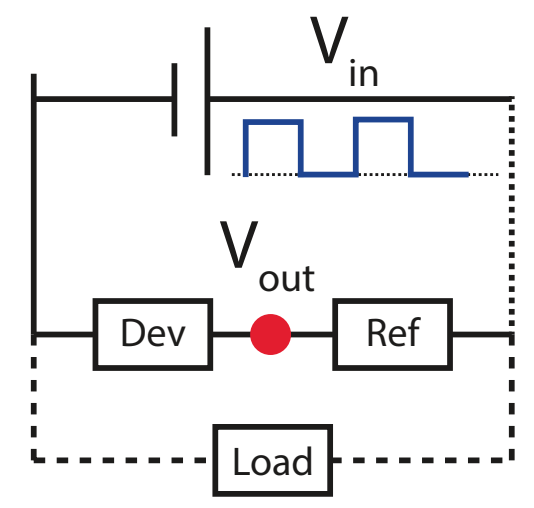

(c)
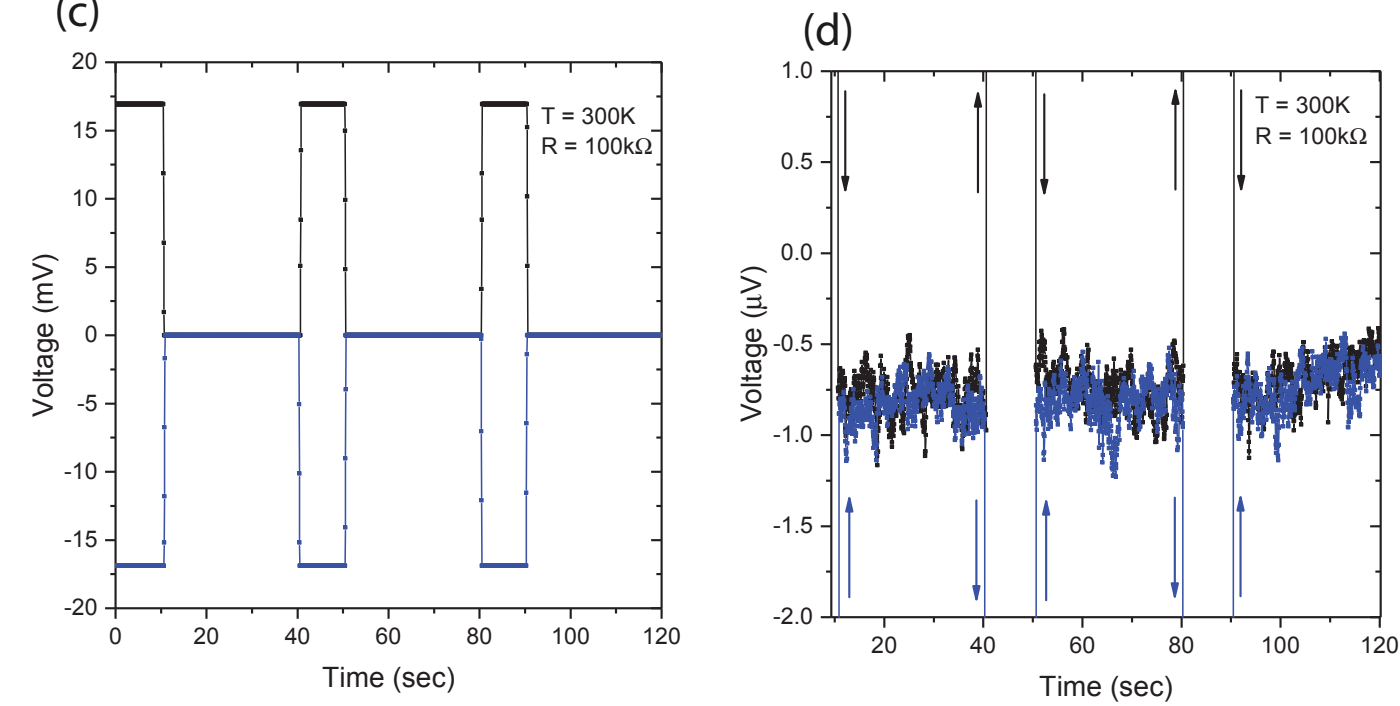

Figure 4.1: (a) Illustration of the experimental set-up. The niobium contacts (blue) are spaced a distance $d$ over the BSTS (gray). The red spheres represent spin-momentum locked charge carriers. (b) Schematic of the DC circuit. The dotted lines can be connected and disconnected to charge the device, or discharge over a $100 \mathrm{k} \Omega$ load resistor. (c) DC measurements performed at $300 \mathrm{~K}$. The black (blue) line is a positive (negative) current process. The large voltage level is the charging step. This is followed by holding, discharge, and hold again. (d) Small voltage zoom of the data in figure (b). 
Chapter 4. Upper limit to the energy retention in the 3D topological insulator BiSbTeSe 2

in figure 4.1(c) and (d) shows the results of this experiment for a positive charging current. The blue line is a repetition of the experiment for a negative charging current. It can clearly be seen that the finite holding voltage is the same for both charging steps. If there is any voltage generated by the spin battery it must either be smaller than the noise of $0.5 \mu \mathrm{V}$ or it decays on a timescale smaller than 0.1 second, putting an upper bound on the performance of the device. Mert Bozkurt et al. have modeled that the equilibrium voltage should depend linearly on the temperature, since the thermal energy is what causes the nuclear spins to relax [160]. The experiment in figure 4.1 is therefore performed at room temperature, which should generate the largest possible signal, even though thermally excited bulk carriers can aid the discharging of the battery. The DC experiment has also been performed at liquid helium temperatures and one obtains the same graphs.

\subsection{AC Measurements}

On the one hand, based on the experiments performed by Tian et al. there is no reason to expect an extremely fast decay of the equilibrium voltage [159]. On the other hand, the nuclear dynamics processess occur typically at a timescale of $\tau_{m}=1 / \gamma_{0} \Gamma_{T}$, where $\gamma_{0}=\lambda^{2} / 8 \pi \hbar^{2} v_{F}^{2}, \lambda=A_{0} v_{0} / S$, and $\hbar \Gamma_{T}=\left(\mu_{L}-\mu_{R}\right) \operatorname{coth}\left(\frac{\mu_{L}-\mu_{R}}{2 k_{B} T}\right)[160]$. In these equations $A_{0}$ is the average hyperfine coupling energy, $v_{0}$ is the unit cell volume, $S$ is the cross section of the helical edge states, and $v_{F}$ the Fermi velocity. $A_{0}$ can be determined by taking the average of all hyperfine coupling constants for every orbital level. Since the bismuth atom is the heaviest of the four in BSTS it will dominate not only SOC, but also the hyperfine interaction and we can limit the calculation to only the bismuth levels. Following Koh and Miller the average hyperfine interaction strength for bismuth is determined to be 62 $\mu \mathrm{eV}$ [161]. From the lattice constants and crystal structure of $\mathrm{Bi}_{2} \mathrm{Te}_{3}$ the unit cell volume is calculated to be $0.5 \mathrm{~nm}^{3}$ [162]. The theory of Metz Bozkurt et al. is applicable to $1 \mathrm{D}$ edge channels ${ }^{3}$ and so the edge state is estimated to be a tube with a radius of $1 \mathrm{~nm}$. The cross-section is then $2 \pi$ $\mathrm{nm}^{2}$, giving

$$
\lambda=A_{0} v_{0} / S=7.89 \cdot 10^{-34} \mathrm{Jm}
$$

\footnotetext{
${ }^{3}$ At the time of writing there is no theoretical prediction for energy storage in $3 \mathrm{D}$ TIs or Weyl/Dirac semimetals that I am aware of
} 
and using the Fermi velocity, estimated to be $5 \cdot 10^{5} \mathrm{~m} / \mathrm{s}$ [93], we find

$$
\gamma_{0}=\lambda^{2} / 8 \pi \hbar^{2} v_{F}^{2}=0.1 \cdot 10^{-10} .
$$

Taking a chemical potential difference of $1 \mathrm{meV}$ between the left and right contact (see appendix A for the influence of changing this chemical potential difference) we can calculate the scattering rate of the charge carriers at the temperature of the experiment, typically $300 \mathrm{~K}$,

$$
\Gamma_{T}=\frac{\left(\mu_{L}-\mu_{R}\right)}{\hbar} \operatorname{coth}\left(\frac{\mu_{L}-\mu_{R}}{2 k_{B} T}\right)=8 \cdot 10^{13} \mathrm{~s}^{-1} .
$$

Finally, the characteristic timescale for nuclear polarization dynamics is

$$
\tau_{m}=1 / \gamma_{0} \Gamma_{T}=1.3 \mathrm{~ms} .
$$

Although $\tau_{m}$ is not the actual decay time of a voltage generated by the spin battery it provides an estimate of the timescales we are dealing with and it explains why the DC measurements that sample every $100 \mathrm{~ms}$, do not show any evidence of retention on BSTS. Using an oscilloscope we can sample the voltage at a much higher rate. A square wave that alternates between zero and a high voltage is applied to the sample while the voltage over the the device is measured and averaged over 512 readings using an oscilloscope. An example of a measurement and the corresponding fit is shown in figure 4.2(a), where the black circles are the measured voltages. The red solid line is the best fit to the data using an exponential function. For this fit the decay time, or RC-time, of the circuit is used as a fitting parameter.

Any physical measurement set-up will yield a finite decay of the voltage no matter how hard one tries to limit the presence of any capacitance in the circuit. The challenge in the $\mathrm{AC}$ measurements of the spin batteries is to show that a measured capacitance can not be attributed to the set-up or other effects unrelated to the retention of spin in the TI flake. For this purpose the measurement set-up has been calibrated using three known resistors, that were wired and measured in the same way as the TI devices. The obtained decay times are plotted as a fuction of the resistance as black circles in figure 4.2(b). The measurements on three test resistors lie on the same straight line within the error margins. The dashed black line is the least squares linear fit that crosses the origin. The fact that all test devices share the same capacitance indicates that we are probing a stray capacitance in the circuit, which makes sense, because the test devices are 
Chapter 4. Upper limit to the energy retention in the 3D topological insulator $\mathrm{BiSbTeSe}_{2}$
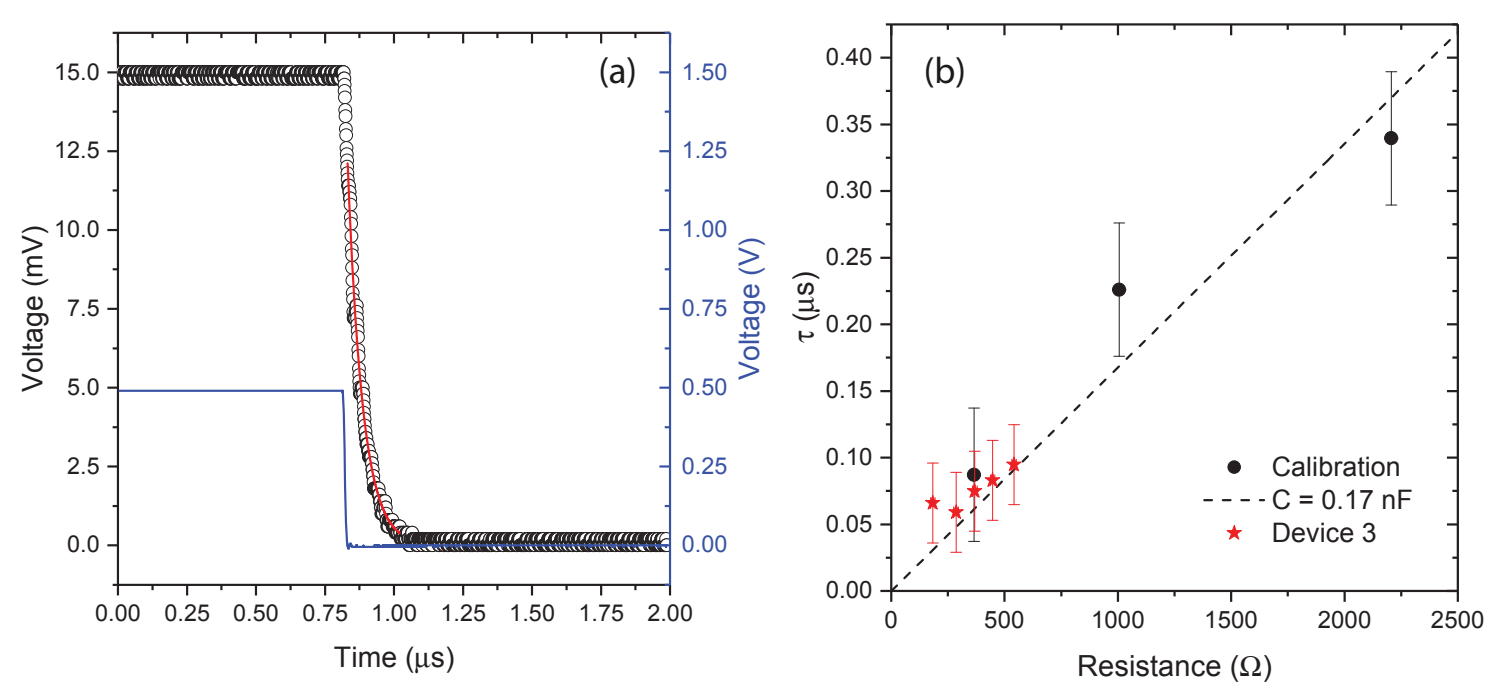

Figure 4.2: (a) Example of an AC measurement. The blue line is the applied current. The black circles show the raw data. The exponential fit is shown by the red line. (b) Extracted RC-times $\tau$ as a function of the total resistance in the cirucit. The black circles are calibration measurements on test resistors and the dashed line is a least squares fit through the origin corresponding to a capacitance of $0.17 \mathrm{nF}$. The red datapoints lie on the dashed line indicating that device 3 exhibits no additional capacitance.

simple resistors. Since $\tau=R C$, the slope of this curve is equal to the stray capacitance that is present in the set-up: $\mathrm{C}_{\text {stray }}=0.17 \mathrm{nF}$. The red stars in figure $4.2(\mathrm{~b})$ show the extracted decay times for BSTS device 3. Since this device has multiple channels of different length, it produces multiple datapoints in this graph that vary in resistance. The observed $\tau$ is four orders of magnitude lower than the estimate in equation 4.4. Although the exact origin of this discrepancy at this time can not be singled out, let alone quantified, it stands to reason that the $3 \mathrm{D}$ nature of the experiment plays an important role. The additional dimension in the surface state allows for scattering mechanisms that do not polarize the nuclear spins, yielding a smaller polarization. The presence of bulk states, for example, allows for the loss of spin information through the Dyakonov-Perel mechanism. Furthermore, a contribution of the bulk to the transport acts as a shunt to the surface state conduction. All in all, moving towards 2D TIs should see a significant increase in the observed decay time. 


\section{Temperature dependence}

Another way to reduce the loss of spin information is to cool down the devices. Not only will this suppress processes such as the Dyakonov-Perel mechanism, it should also slow down the relaxation of the nuclear spins, thereby increasing the observed decay time $\tau$. Device 3 was cooled down in a flowcryostat that allows us to control the temperature between room temperature and $4 \mathrm{~K}$. The AC measurements were repeated at different temperaures during the cooldown. Figure 4.3 shows the extracted decay times (black) and the resistance of the channel (red), as a function of temperature. Between $220 \mathrm{~K}$ and $300 \mathrm{~K}$ the device shows semiconductor-like behavior; a resistance that increases with decreasing temperature. Below $220 \mathrm{~K}$ the temperature dependence becomes metallic; decreasing with decreasing temperature. This cross-over around $220 \mathrm{~K}$ indicates the transition from a bulk-dominated transport to transport that is governed by the metallic surface states, which is typical of a TI. At the lowest temperatures the niobium leads become superconducting, which translates to a sharp decrease of the resistance. Partly because the leads itself have zero resistance, but mostly because the proximity effect effectively shortens the BSTS channel. The extracted $\tau$ follows the same temperature dependence as the resistance of the device, as can be seen in figure 4.3. This shows that the change in $\tau$ can be fully attributed to a change in resistance, and that the total capacitance of the circuit remains unchanged. This observation solidifies the fact that the tested devices do not add an additional capacitance to the circuit.

In short, we have fabricated three BSTS devices and studied their retention of energy in both slow (DC) and fast (AC) measurements. An open circuit voltage in a DC measurement would be our smoking gun, showing that the devices behave as a battery. These measurements, however, showed no open circuit voltage within the measurement noise. Sampling the voltage at a much larger rate allows us to track the decay of the voltage across the devices as a function of time. The observed decay time is comparable to the test resistors, indicating that the devices do not store and release energy. Cooling down the devices does not change the capacitance associated with the BSTS devices. A more extensive model of the energy retention in 3D TIs is required to understand this low upper limit to the battery perfomance of the devices. Moving towards the 1D limit, which can already be understood theoretically, should see a significant increase in the capacitance that can be observed in our measurement set-up. It also allows one to control the bulk contribution to the transport by applying a gate electric field, thereby 
Chapter 4. Upper limit to the energy retention in the 3D topological insulator $\mathrm{BiSbTeSe}_{2}$

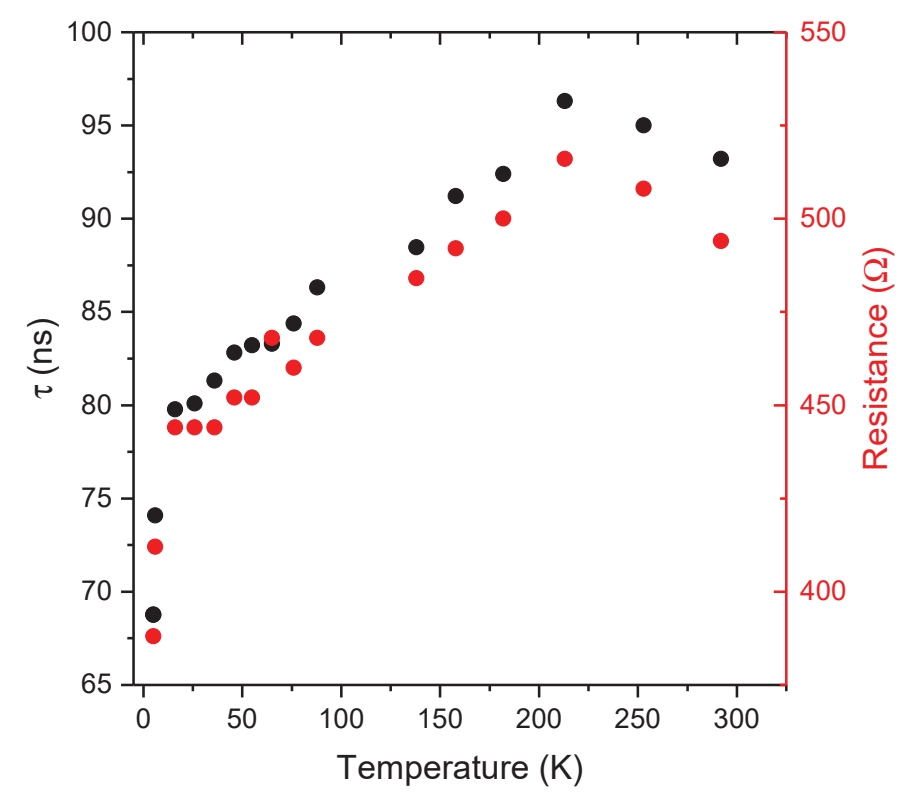

Figure 4.3: Temperature dependence of the RC-time $\tau$ (black) and the resistance (red) of device 3 . The curve shows semiconductor-like behavior above $220 \mathrm{~K}$, which changes to metallic behavior below $220 \mathrm{~K}$. This cross-over is indicative of a topological insulator that has its Fermi level in the bulk bandgap. At low temperatures the superconducting transition of niobium creates a sharp decrease in resistance.

enhancing the spin battery performance. 


\section{Appendix A: Scattering rate}

The applied voltages in the experiments presented in this chapter do not exceed $10 \mathrm{mV}$. Figure A.1 shows a plot of $\Gamma_{T}$ as a function of the voltage difference. This is equation 4.3 of this work and was written by Mert Bozkurt et al., equation 10. In the range of experimentally realistic voltages there is virtually no change in $\Gamma_{T}$ and thus in the observed decay time.

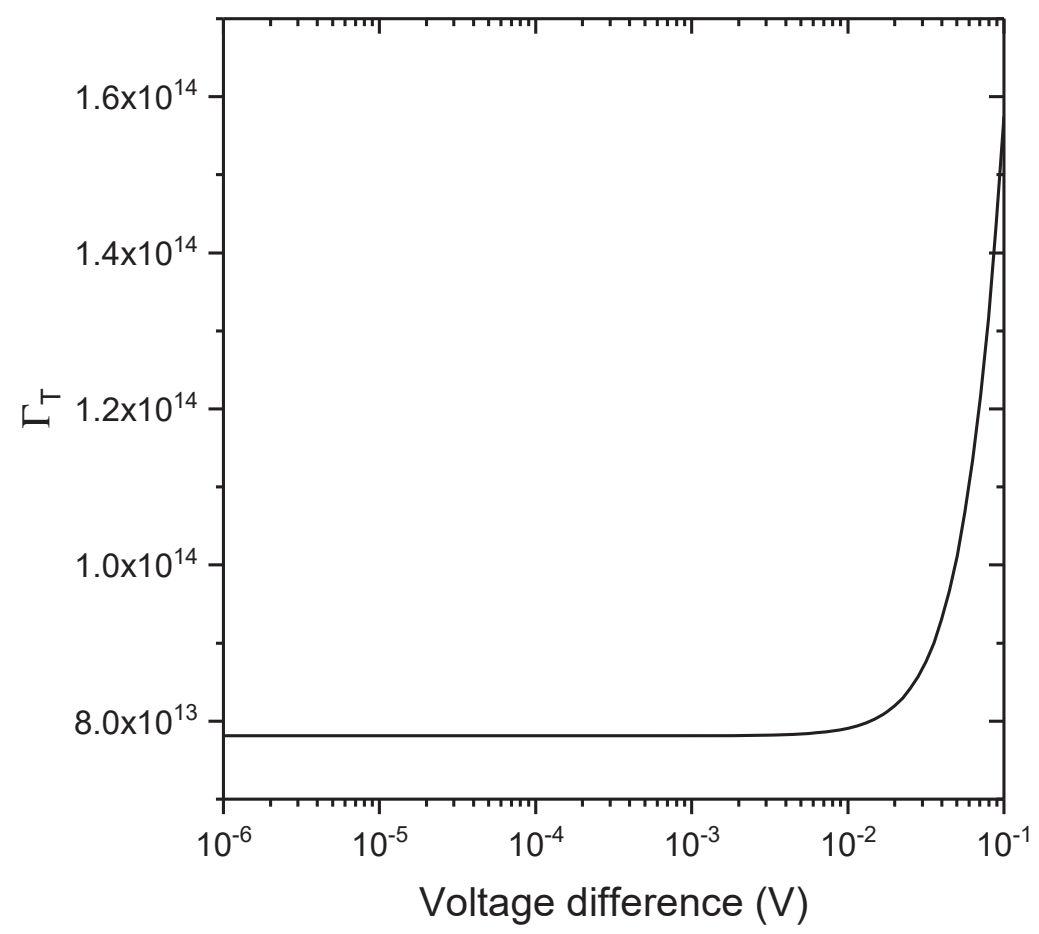

Figure A.1: Scattering rate as a function of the applied voltage as given by equation 4.3. For voltages below $10 \mathrm{mV}$ the effect of changing the charging voltage is negligable. 


\section{Chapter}

\section{Origin of the butterfly magnetoresistance in $\mathrm{ZrSiS}$}

ZrSiS has been identified as a topological material made from nontoxic and earth-abundant elements. Together with its extremely large and uniquely angle-dependent magnetoresistance this makes it an interesting material for applications. In this chapter I study the origin of the so-called butterfly magnetoresistance by performing magnetotransport measurements on four different devices. The butterfly $M R$ is attributed to perfect electron-hole compensation in the two-band Drude model, generated by the Zeeman-effect. Furthermore, the observed Shubnikov-de Haas oscillations are carefully analyzed using the Lifshitz-Kosevich equation to determine their Berry phase and thus their topological properties. Although a link between the butterfly $M R$ and the Berry phase remains uncertain, the topological nature of ZrSiS is shown. 


\subsection{Introduction}

ZrSiS belongs to a class of electronic materials known as nodal-line semimetals $[25,107]$. These materials are distinct from topological insulators (TI) and Dirac/Weyl semimetals (DSM/WSM) in that the touching of the bands occurs not in one place, but in lines or circles [164-168]. A number of studies have reported on the enormous, nonsaturating magnetoresistance (MR) that $\mathrm{ZrSiS}$ displays $[108,110,169,171,216]$, a feature that makes $\mathrm{ZrSiS}$ a good candidate for sensor applications, for example. The general usability of $\mathrm{ZrSiS}$ is supported by its stability and elemental abundance. The material contains three non-toxic and ubiquitous elements and shows no signs of degradation whatsoever $[107,109]$. Not only is the MR in ZrSiS extremely large, it has a peculiar angular dependence as well, showing a maximum MR when the angle between the applied magnetic field and the sample surface is $45^{\circ}$, instead of a perpendicular field as one would expect. When plotted, the graph has a distinct butterfly shape and so the effect has been christened butterfly magnetoresistance[110, 169, 172, 216]. Although a lot has been written about both the experimental side of the large butterfly $\mathrm{MR}$ and the theoretical side examining the intricate band structure from which the transport properties arise $[25,107,168,173,174]$, a clear link between the two has not yet been established. Due to the high mobility of the charge carriers in $\mathrm{ZrSiS}$, studying quantum oscillations provides us with a great opportunity for understanding the unique transport properties of this material, as illustrated by the number of reports on quantum oscillations in $\mathrm{ZrSiS}$ [111, 175, 176, 189]. In this chapter I study the origin of the butterfly magnetoresistance. To this end I find the charge carrier density and mobility of four different $\mathrm{ZrSiS}$ thin flakes, as well as their dependence on the angle of the magnetic field. This is followed by an analysis of the measured Shubnikov-de Haas oscillations, which together lead to a possible explanation of the butterfly MR. The SdH also provide an opportunity to study whether or not any topological properties of the nodal line in $\mathrm{ZrSiS}$ shine through.

\subsection{Butterfly magnetoresistance}

To create the devices, I have exfoliated flakes of two different ZrSiS crystals. Crystal 1 was made by Yingkai Huang at the University of Amsterdam. Crystal 2 was made by Leslie Schoop at the Max Planck Institute for Solid 

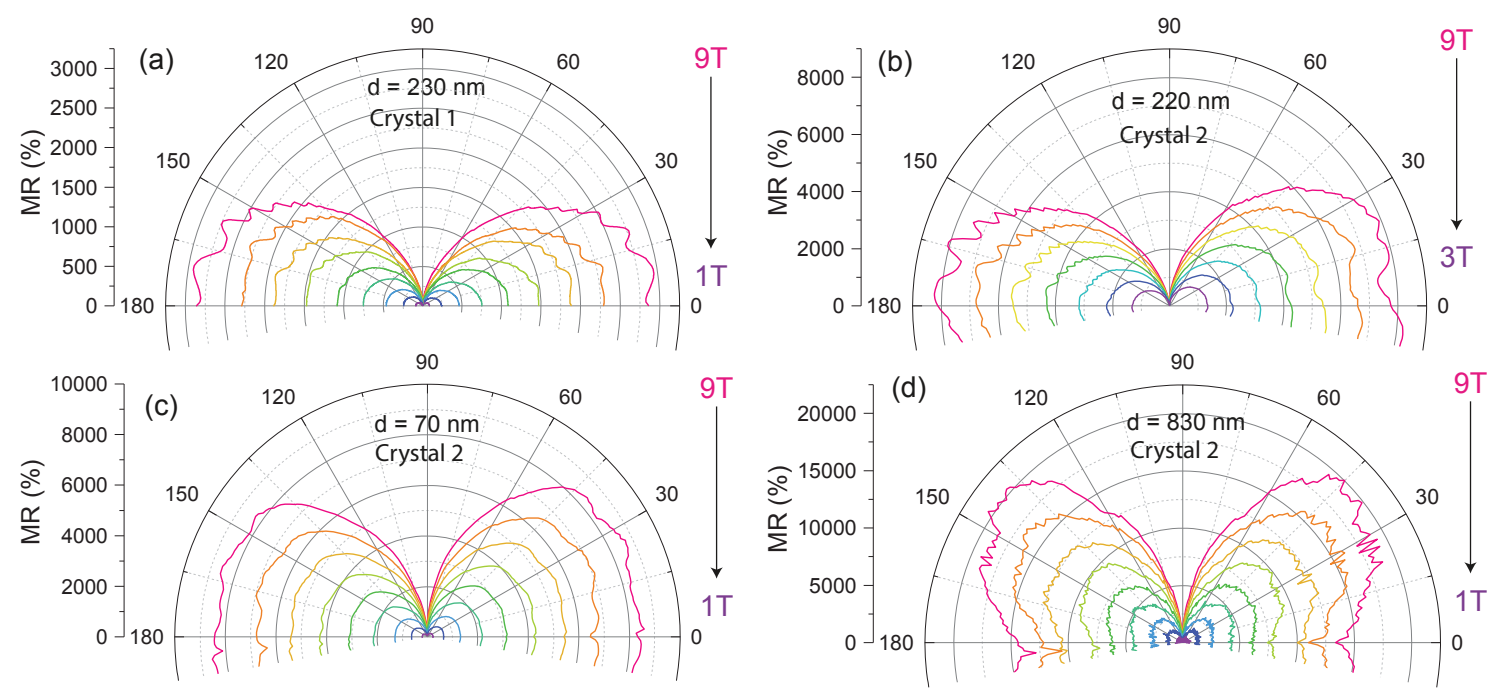

Figure 5.1: Angle-dependence of the MR of two samples made from different ZrSiS crystals, where 0 degrees is out-of-plane. Device (a) is made from a $230 \mathrm{~nm}$ thick flake of crystal 1 . The MR does not exceed $2500 \%$ and the angle-dependence of the MR has a maximum at 0 degrees. (b) ADMR of a $220 \mathrm{~nm}$ thick flake of crystal 2. The MR has a maximum around 20 degrees. (c) ADMR of a $70 \mathrm{~nm}$ flake of crystal 2 showing a flat maximum MR of $8000 \%$ between 0 and 45 degrees. (d) ADMR of the $820 \mathrm{~nm}$ thick flake of crystal 2. The MR reaches values of $20.000 \%$. The angle-dependence of the MR is clearly butterfly-shaped, having a maximum at 45 degrees.

State Research in Stuttgart. Due to the crystal structure of ZrSiS there is a natural cleavage plane between the sulfur layers, as is shown in detail in section 2.2. All exfoliated flakes therefore show the (001)-plane as their top surface [107]. The flakes were deposited on a Si substrate with a 300 nm thick $\mathrm{SiO}_{2}$ top layer. After determining the thickness of the flake using AFM, gold contacts were designed and deposited on the flakes using e-beam lithography and RF sputtering. In this work we describe measurements performed on four standard six-probe devices made in this fashion. The first of these uses a flake from crystal 1 , the other three have flakes from crystal 2. I have studied further devices based of crystal 1 including a bulk devices contacted with gold wires and silver epoxy. All of these measurements showed similar results and are therefore left out of this chapter.

The devices were cooled down to $2 \mathrm{~K}$ in a Quantum Design Physical Property Measurement System (PPMS) on an insert that can be rotated 180 degrees in a maximum magnetic field of $9 \mathrm{~T}$ while recording the longitudinal and transversal voltage in the device in a four-probe configuration, using a 
$100 \mu \mathrm{A}$ current. Here $0^{\circ}$ and $180^{\circ}$ are defined as an out of plane magnetic field. Figure 5.1 shows the angle-dependent magnetoresistance (ADMR) of all four devices. The MR is calculated as $100 \% \times[R(H)-R(0)] / R(0)$. The results for the different devices mainly differ on two points: the size of the MR and the shape of the angle-dependence of the MR. Although slightly obscured by the Shubnikov-de Haas oscillations that are present for high magnetic fields and angles close to 0 , the maximum MR in figure 5.1(a) is observed at $0^{\circ}$ and $180^{\circ}$, as one would expect for a regular thin conductor. The maximum MR in figure 5.1(d) lies around $45^{\circ}$, a feature that is known as the butterfly MR. The size of the magnetoresistance varies by a factor of 8 between the two graphs. The flake from the first crystal of $\mathrm{ZrSiS}$, figure 5.1(a) has an MR of about $2500 \%$, whereas the flake from the second crystal of ZrSiS shows an MR exceeding $20.000 \%$. The angle-dependent MR of the $220 \mathrm{~nm}$ and $700 \mathrm{~nm}$ thick devices is plotted in figure 5.1(b) and 5.1(c) respectively. Both the $70 \mathrm{~nm}$ and $220 \mathrm{~nm}$ thick device do not show such a clear butterfly-shaped MR as the $830 \mathrm{~nm}$ thick device does. Their maximum MR is 2.5 times lower, around $8000 \%$. Furthermore, this maximum is not clearly located at $45^{\circ}$, as it is in figure $5.1(\mathrm{~d})$. For the $220 \mathrm{~nm}$ thick device, figure 5.1(b), the maximum MR at $45^{\circ}$ is absent altogether. The $70 \mathrm{~nm}$ thick device, figure 5.1(c), does reach the maximum at $45^{\circ}$, but keeps this value towards $0^{\circ}$ (perpendicular field) instead of decreasing. It effectively displays a sum of the regular ADMR and the butterfly MR. Comparing all measurements we can conclude that crystal 2 exhibits a butterfly MR similar to what was observed by Ali et al. [110].

\section{The origin of the butterfly}

Having observed a butterfly MR in some, but not all samples, provides a great opportunity to study this anomalous version of the extremely large MR. Such a large increase in resistance can be found in other materials as well and can be caused by a near perfect balance between electron and hole densities in the two-band Drude model $[169,181,182]$. In this model the longitudinal resistivity is given by

$$
\rho_{x x}(B)=\frac{1}{e} \frac{\left(n_{h} \mu_{h}+n_{e} \mu_{e}\right)+\left(n_{h} \mu_{e}+n_{e} \mu_{h}\right) \mu_{h} \mu_{e} B^{2}}{\left(n_{h} \mu_{h}+n_{e} \mu_{e}\right)^{2}+\left(n_{h}-n_{e}\right)^{2} \mu_{h}^{2} \mu_{e}^{2} B^{2}}
$$

For large fields the rightmost terms of the numerator and denominator dominate the leftmost terms, so we can focus on them. From the denominator it is clear that when the hole and electron density become equal the 
resistivity has a maximum. The exact value of the resistance and the MR still depends on the mobilities of the two bands. Figure 5.2 shows the fit of the two-band Drude model to the longitudinal and transverse conductivity of the two most distinct devices. Figure 5.2(a) shows the data and the best fit to the data of the device made from crystal 1 , which exhibits the lowest MR and no butterfly MR. Figure 5.2(b) shows the data and fit of the $830 \mathrm{~nm}$ thick sample made from crystal 2. This sample shows strong anisotropic ADMR and a much larger MR in general. Comparing the two panels of figure 5.2 tells us that in both cases the electron and hole density are nearly compensated, indicated by the $\sigma_{x y}$ that tends to zero for large magnetic fields. These measurements support the idea that electron-hole compensation is the mechanism that generates the enormous magnetoresistance in ZrSiS. To understand the connection to the butterfly MR, the two-band model is applied to the longitudinal and transverse MR obtained at different angles between the sample and the magnetic field. The model was unable to accurately describe the data obtained at $30^{\circ}$, which is why these fitting parameters have been left out. The result of all other fits is presented in figure 5.3(a). The black and red curve describe the electron and hole carrier density respectively. The angles where the MR peaks, around $45^{\circ}$, are also the angles where the electron and hole densities cross in figure $5.3(\mathrm{a})$, further corroborating the explanation of the butterfly MR in terms of near-perfect electron-hole compensation.

In principle, rotating a sample should not alter the carrier density. The fact that a change in carrier density is observed in figure 5.3(a) can, however, be understood in a magnetic field. Besides the formation of cyclotron orbits typically associated with magnetoresistance, the magnetic field also induces a Zeeman shift in the energy levels. The Zeeman effect can, in a complex band structure such as that of ZrSiS, slightly alter the size and shape of the Fermi pocket and thereby alter the carrier density of the material. Figure 5.3(b) shows the extracted fitting parameters for different ranges of the perpendicular magnetic field. It is clear from this figure that the field is able to tune the carrier density. The carrier density highly depends on the exact crystal parameters, such as the position of the Fermi level, which is likely the reason why the butterfly is not observed in all devices. Contributions of the Zeeman effect to an extremely large MR have previously been identified in $\mathrm{MoSi}_{2}$ [182], $\mathrm{WTe}_{2}[183,184]$, and $\mathrm{Cd}_{2} \mathrm{As}_{3}$ [185]. 

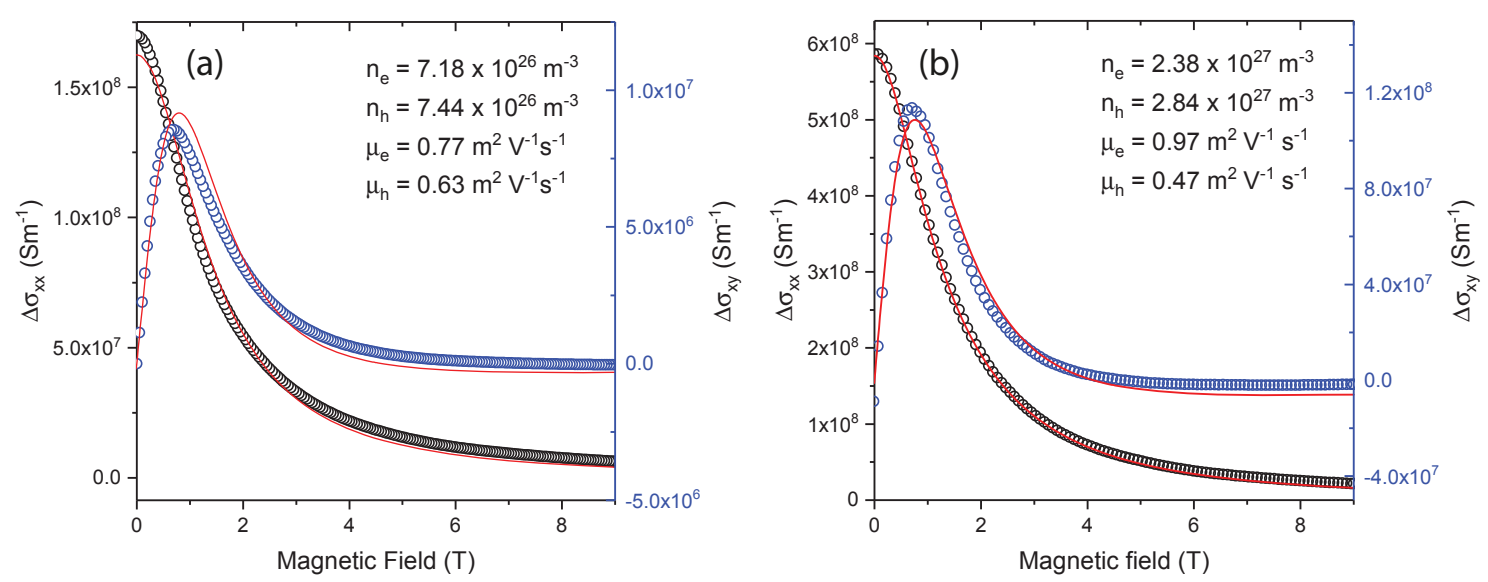

Figure 5.2: Two-band Drude fits (red lines) of the longitudinal (black circles) and Hall (blue circles) conductivity for two devices measured using a bias current of 100 $\mu \mathrm{A}$. All fitting parameters are indicated in the top-right corner. (a) Measurement data of the $230 \mathrm{~nm}$ thick flake of crystal 1. (b) Measurement data of the $830 \mathrm{~nm}$ thick flake of crystal 2.
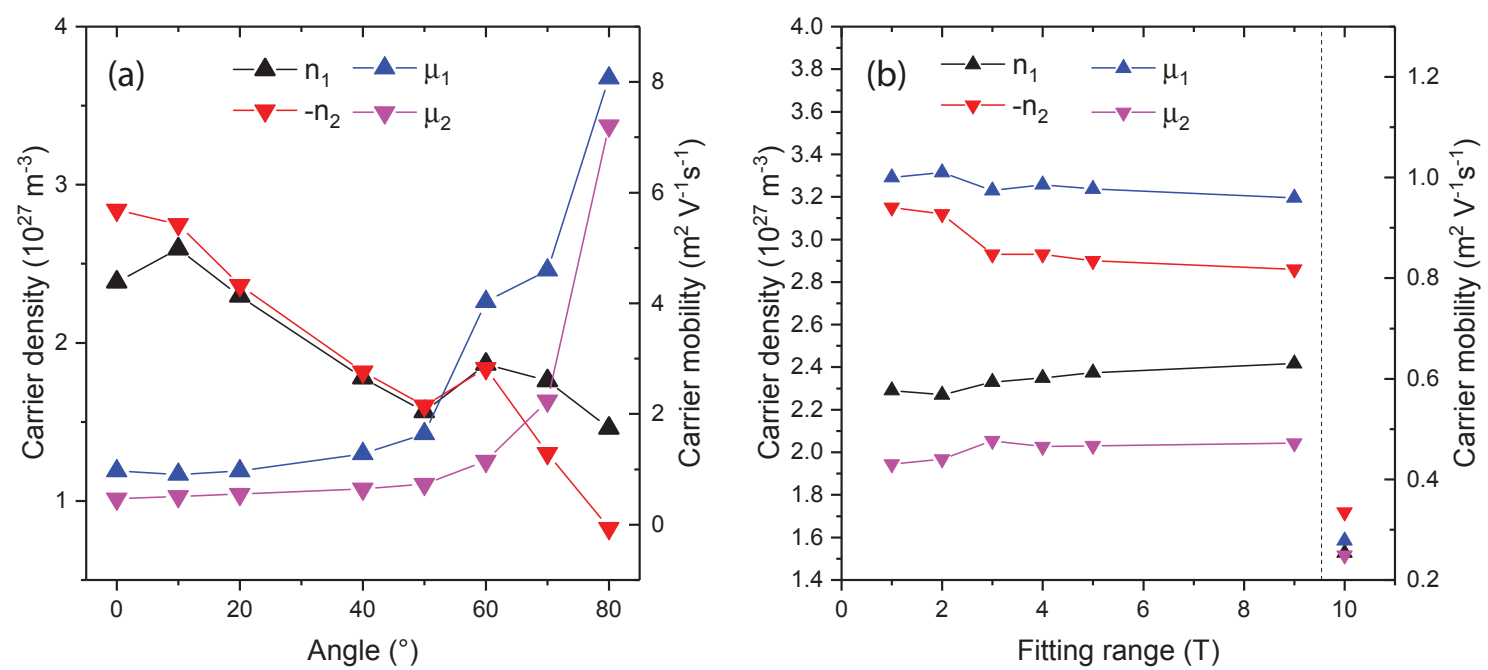

Figure 5.3: Carrier densities and mobilities extracted from two-band Drude fits on the longitudinal and Hall resistance of the $830 \mathrm{~nm}$ thick flake of crystal 2. (a) Parameters for different angles between the sample and the magnetic field. Only the out-of-plane component of the magnetic field has been used. (b) Fit parameters for different fit ranges of the perpendicular field ( $0 \mathrm{~T}$ up to the indicated value), showing that the magnetic field influences the carrier density. The datapoints indicated to be $10 \mathrm{~T}$ are separated by the dashed line and belong to fits performed for the range $\mathrm{B}=5 \mathrm{~T}$ to $9 \mathrm{~T}$. 


\subsection{Shubnikov-de Haas oscillations}

To distill the $\mathrm{SdH}$ oscillations for the longitudinal magnetoresistance, the measured voltage was symmetrized. The background was subtracted from the signal by smoothening the oscillations. Figure 5.4 shows the fast Fourier transforms of all the oscillations. The data has been offset for clarity and for some larger angles the data has been amplified for visibility, as shown by a factor after the angle indication. Two distinct peaks can be identified at the perpendicular orientation $\left(0^{\circ}\right)$, located at frequencies of $16 \mathrm{~T}$ and 243 T. These SdH frequencies are well known in literature [109, 110, 175, 216], and their position as a function of the angle between the sample and the magnetic field is a clear indicator of the butterfly MR. This link is clear from comparing figures 5.1 and 5.4, and has been observed in literature [110]. The oscillation with a frequency of roughly $16 \mathrm{~T}$ does not move as a function of the angle, indicating that the Fermi pocket it corresponds to is spherical. The Onsager relation tells us the extremal cross-section, $S$, of the Fermi pocket that is responsible for an oscillation [186],

$$
S=\frac{2 \pi e F}{\hbar},
$$

where $F$ is the frequency of the oscillation in Tesla. For a $16 \mathrm{~T}$ oscillations this gives a reciprocal area of $1.53 \times 10^{-3} \AA^{-2}$. The peak that starts at $F=243 \mathrm{~T}$ in all panels of figure 5.4 corresponds to a reciprocal area of $1.53 \times 10^{-3} \AA^{-2}$. Both these frequencies are in good correspondence with literature $[110,111,175]$. The $3 \mathrm{D} \mathrm{BZ}$ of $\mathrm{ZrSiS}$ is a fourfold symmetric structure that has two distinct features. Section 2.2 includes two illustrations of the $\mathrm{BZ}$ from literature as a reference to the reader. In the $\Gamma Z$ direction one finds elongated tubular shapes that extend in the $k_{z}$ direction. They have also been named 'petals', because viewed from the top they look like petals on a flower. These tubular shapes are hole pockets and have an extremal cross-section that corresponds well with a SdH-frequency of $243 \mathrm{~T}$. The other main feature is an electron-pocket situated in the $\Gamma M$ direction. They are known as the 'diamond' or 'dog-bone' feature and have a larger extremal cross-section than the tubes, corresponding to a SdH-frequency between 550 and $600 \mathrm{~T}[111,175]$. The smallest reciprocal area, corresponding to the $16 \mathrm{~T}$ peak, is more difficult to link to the Fermi surface. Depending on the Fermi level some smaller pockets may connect to or be separated from the two main features, especially the tubular hole-pocket. These small pockets are a likely origin of the low frequency oscillation. Another possible explanation is that the holes circle around the smallest part 
of the tube. Depending on the position of the Fermi energy the tubes may stretch through the entire $\mathrm{BZ}$ or be cut off around the $k_{x^{-}} k_{y}$ plane. As long as the Fermi surface at the edge of the tube is not closed, but has a hole in it, this provides another extremal cross-section. This cross-section is not the largest, but the smallest loop a carrier can make. The BZ calculated by Pezzini et al. [111] shows a fully connected tubular hole-pocket and the Fourier spectrum of their SdH-oscillations does not show a peak around 16 $\mathrm{T}$, supporting this idea.

The behavior of the $243 \mathrm{~T}$ peak with the angle between the sample and the magnetic field is a clear indicator of the presence of butterfly shaped MR. Figures 5.4(a) and (b) both show the peak moving towards larger frequencies, indicating that the extremal cross-section increases in size. A perfectly cylindrical Fermi surface would give a $\cos (\theta)$-dependence of the frequency. By treating the tubular hole-pocket as an ellipsoid we can fit the aspect ratio of this ellipsoid. Doing so yields a ratio of 2.2 for the two ellipsoid axes for figures 5.4(a) and (b). The $F(\theta)$ dependence of the 243 $\mathrm{T}$ peak displayed in figures 5.4(c) and (d) is quite the opposite of that in figures 5.4(a) and (b). Although figure 5.4(c) still faintly exhibits a right moving peak, both (c) and (d) clearly show that from $30^{\circ}$ onwards the peak splits and moves to lower frequencies. This is in perfect correlation with the appearance of the butterfly magnetoresistance to the point that the thinnest device, presented in figure 5.1(c) and figure 5.4(c), shows a mixture of regular and butterfly angle-dependence in both its MR and $\mathrm{SdH}$ oscillations. From this comparison it is clear that the hole-pocket plays an important role in the appearance of the butterfly MR. A Zeeman shift of this pocket by itself will not yield a change in the carrier density and thus no butterfly MR. Most likely an interaction of this hole-pocket with the larger electron-pocket takes place. The corresponding SdH frequency has been studied in literature [111, 175], but is too large to be reliably measured in our set-up. Albeit faintly, a corresponding peak can be seen in some of the Fourier spectra, such as the $10^{\circ}$ lines of figure $5.4(\mathrm{~b})$ and $(\mathrm{d})$.

\subsection{The Lifshitz-Kosevich model}

Quantum oscillations in materials, such as the Shubnikov-de Haas oscillations observed here in $\mathrm{ZrSiS}$, can be described with the model created by Lifshitz and Kosevich in 1956 [187, 188]. Appendix A contains a detailed description of the model used in this section. A careful analysis of data 

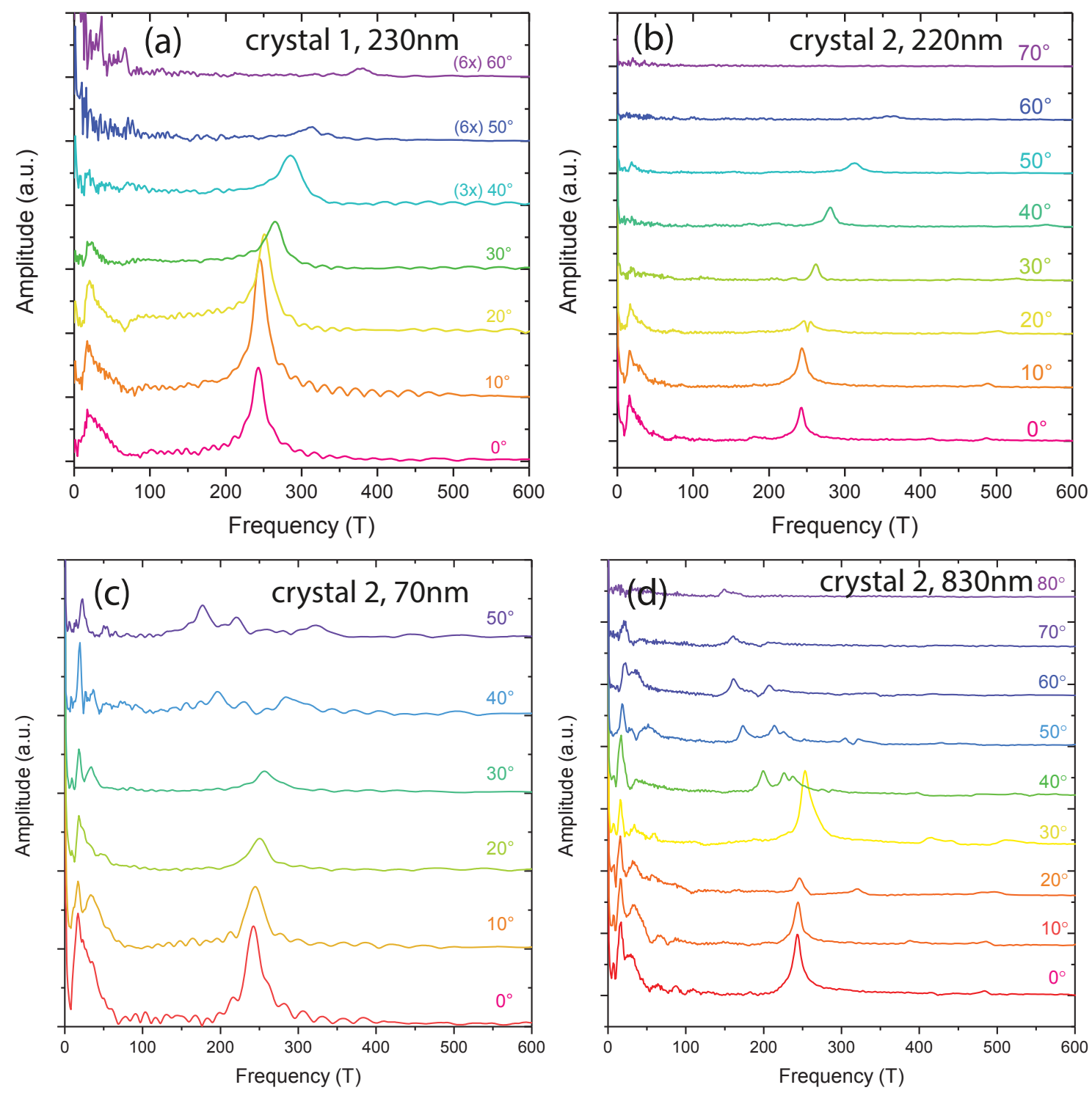

Figure 5.4: (a)-(d) Fourier spectra of the Shubnikov-de Haas oscillations measured at different sample orientations. The data is offset for clarity. The angle between the sample and the magnetic field is indicated in the graphs for each line. The thickness of the device on which data has been taken is indicated on the top of each graph. Figures (a) and (b) show the $243 \mathrm{~T}$ peak moving towards higher frequencies with increasing angle. These devices do not exhibit the butterfly-shaped MR. Figures (c) and (d) each show two peaks moving towards lower frequencies for angles larger than $30^{\circ}$. These devices do show butterfly-shaped MR. 
allows us to extract the phase of the SdH-oscillations and can then tell us whether or not the charge carrier has picked up an additional phase along its trajectory. The Lifshitz-Kosevich (LK) model can thus be used to determine whether or not that particular path through the BZ is accompanied by a Berry phase $\phi_{B}$, which is a clear signature of topological transport. Furthermore, the phase of the quantum oscillations is influenced by the carrier type, the dimensionality of the band, and the dispersion of the band (linear of parabolic). In a recent publication, $\mathrm{Li}$ et al. have collected all possible phase shifts in the LK model for a topological nodal-line semimetal, showing that claiming non-trivial transport is not so trivial [189]. The circular orbit around the hole-populated tube from which the $243 \mathrm{~T}$ oscillation originates is associated with a phase shift of $\delta=0$. They come to the conclusion that this orbit encloses a Dirac point, which should yield a discernable Berry phase in the quantum oscillations. Other investigations into the topological nature of this Fermi pocket have yielded a variety of conclusions, including fully trivial [216], fully topological [111], Berry phases that are neither $\pi$ nor 0 [176], and a Berry phase that changes from trivial to topological for some angles of the magnetic field [110]. These articles have been written before the publication of the theoretical work by Li et al. and have all assumed 3D carriers, whereas Li et al. note that the $243 \mathrm{~T}$ frequency is mostly in the 2D regime [189]. Hyun et al. have studied the Berry phase in materials with a square-net substructure, belonging to the $\mathrm{P} 4 / \mathrm{nmm}$ or Pnma space group, and in ZrSiS specifically [178]. In their work they highlight the tubular holepocket as a "Berry hot spot", a Fermi surface that encloses a large Berry curvature. Importantly, they note that the Berry phase strongly depends on the Fermi energy and electron-hole asymmetry.

Figure 5.5(a) and (b) show, as an example, two typical SdH oscillations measured on the device that exhibits clear butterfly MR. Nearly all of the extracted oscillations are characterized by a beating pattern, which is especially prevalent from $40^{\circ}$ onwards. Modeling these oscillations using the LK model with one frequency is possible, but less than ideal. By applying a bandpass filter around the desired frequency some of the beating can be suppressed. Even then, it is not always possible to fit the full extent of the oscillation, but for angles below $50^{\circ}$, a portion of the data has been fit. The Berry phases obtained by this procedure are shown as black circles in figure $5.5(\mathrm{c})$. The dashed lines serve as a reading guide and indicate $\phi_{B}=\pi$, which is typically associated with topological states. Since there is an obvious beating pattern in the $\mathrm{SdH}$ oscillations a LK model using two 
frequencies is more appropriate. The red lines in figures 5.5(a) and (b) are the best fits to the data using this two frequency model. All relevant fitting parameters are indicated in the top-right of the respective panel. The Berry phases resulting from this analysis have been included in figure 5.5(c). The red and magenta triangles belong to one of the frequencies, while the blue and violet triangles belong to the other. The phases have been drawn as red and magenta to remind the reader of the fact that for angles of $40^{\circ}$ and larger there are several peaks in the $\mathrm{SdH}$ spectrum (figure 5.4(d)). Here, we examine the left-most one of the three.

Beating is caused by the presence two similar, but different, frequencies. If the Zeeman splitting plays an important role in the formation of the butterfly MR, as was suggested in section 5.2, two nearly identical SdH frequencies are to be expected. In general, Zeeman splitting does not change the shape of the two (now non-degenerate) pockets. However, in the complex $\mathrm{BZ}$ of $\mathrm{ZrSiS}$, it is possible that the Zeeman shift pushes two different bands so close to each other that they start to deform, which would show up as a beating pattern between the altered and unaltered pocket. Since the Zeeman effect depends on the strength of the magnetic field, so should the beating frequency. Unfortunately, incorporating this into the LK model, by making the frequency difference depend linearly on $B$, I have not been able to accurately model the $\mathrm{SdH}$ oscillations.

The main feature of figure 5.5(c) is that the obtained $\phi_{B}$ increases with angle. Although it starts out at the trivial state zero for the $1 \mathrm{~F}$ model and $\mathrm{F}_{1}$ of the $2 \mathrm{~F}$ model, the phases increase, alternating between trivial and topological. For clarity the Berry phases have been drawn to go up to $5 \pi$, even though in reality $\phi_{B}$ is $2 \pi$-periodic. At $40^{\circ}$, the angle where the peaks in the Fourier spectrum split, the Berry phase suddenly makes a jump of $1.5 \pi \pm 0.4 \pi$. Generally speaking, it is possible that the Berry phase deviates from the standard cases of zero (trivial) and $\pi$ (topological). These values are only found when the path through the BZ fully encompasses the Dirac node, as for example a spherical pocket does. Any extremal cross-section must then necessarily fully encompass the Dirac node if it is present, hence we find either $\phi_{B}=0$ of $\phi_{B}=\pi$. If we were to saw off a small part of the sphere, and perhaps elongate it into a cigar shape (as is the case in $\mathrm{ZrSiS}$ ), we have created another extremal orbit, one that does not fully enclose the Dirac node and thus encircles an arbitrary amount of Berry connection. In such a situation there can be any $\phi_{B}$ between zero and $\pi$. 
In terms of the spin-texture, only the in-plane projection of the spin makes a full rotation and there is an additional perpendicular component that maintains its value. Considering a spherical Fermi pocket, the Berry phase obtained from the path integral of the Berry connection is the same for all angles of the magnetic field. Although the extremal orbit that generates the $\mathrm{SdH}$ oscillation moves over the sphere so that it lies perpendicularly to field, there will always be a Berry phase of $\pi$. This is also true for a tubular, or cigar-shaped, Fermi pocket, although the extremal loop becomes elliptical upon rotation. Only when we consider a minimal extremal cross-section, such as an open top of a cylinder or the aforementioned sawed-off-sphere, can we expect the Berry phase to change with rotation of the field. Carriers cannot traverse the nicely balanced elliptical orbit through the pocket as before, because the pocket is open on one side. Another possibility is that the pocket deforms due to the Zeeman effect and the crowded bandstructure, as was introduced earlier in this chapter. This can be seen by looking at one of the mathematical expressions of the Berry phase [179, 180].

$$
\phi_{B}=\int_{S} \boldsymbol{\Omega}_{n} d \mathbf{S},
$$

which says that the Berry phase is equal to the amount of Berry curvature, $\Omega_{n}$ that pierces an area $S$. This Berry curvature is analogous to the field lines known from electromagnetism. If (the angle-dependence of) the Zeeman effect changes the shape of the pocket it therefore also influences the Berry phase. It is unlikely that purely this change in Fermi surface morphology is responsible for the angle-dependence of $\phi_{B}$ that was reported in figure 5.5(c). From Hyun et al. and the comparison of this work with other works in literature we know that the topological properties of $\mathrm{ZrSiS}$ are highly sensitive to properties such as the Fermi level and electron/hole ratio $[110,111,175,178,216]$. The latter could be the link between the Berry phase and the butterfly MR, as the importance of the electron/hole ratio was also identified in section 5.2. Upon tilting the magnetic field one could make two pockets touch and fuse together, instantly increasing the size of the loop, $S$, which would show as a sudden jump in Berry phase such as was observed by, for example, Ali et al. [110]. Although a definitive explanation cannot be identified, it is not unlikely that this mechanism is responsible for the jump in Berry phase at $40^{\circ}$ in figure 5.5(c). 

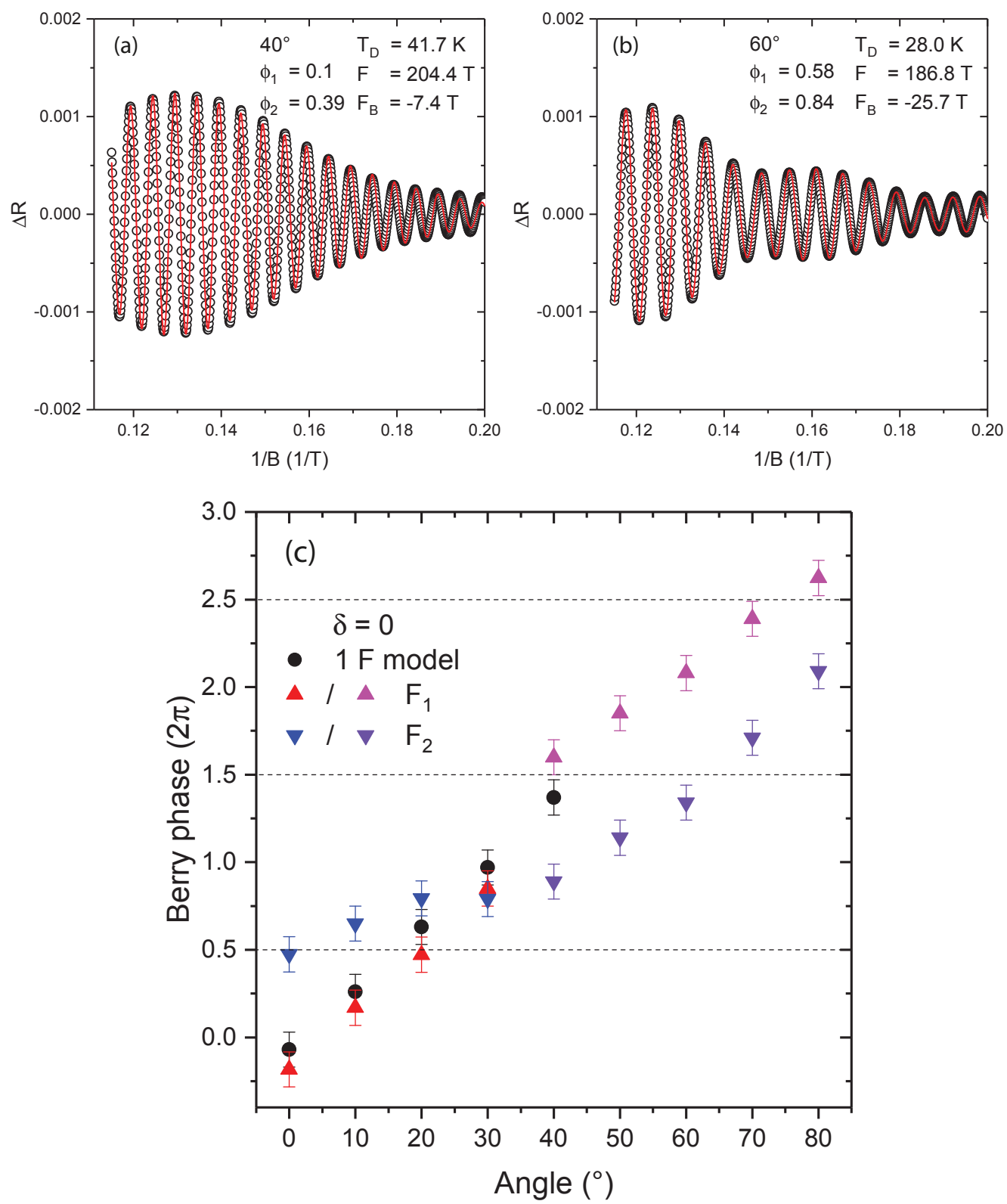

Figure 5.5: (a) and (b) show two examples of the LK model applied to the extracted $\mathrm{SdH}$ oscillations (black circles). The fits (red lines) have been made by adding two frequencies to create the beating pattern. All relevant fitting parameters as well as the corresponding angle are indicated in each panel. $F_{B}$ is the difference between the two used frequencies. (c) Berry phases obtained from the LK fits, using $\delta=0$. The black circles are derived from fits using only one frequency. Red/magenta triangles show $\phi_{B}$ of the first frequency. The blue/violet triangles show $\phi_{B}$ of the second frequency. 


\subsection{Conclusions}

The magnetoresistance in ZrSiS can safely be labeled extremely large. This large MR can be understood by considering a two-band Drude model where the two bands are equally populated by opposite charge carriers. This nearperfect electron-hole compensation may also be the driving mechanism behind the peculiar butterfly-shaped magnetoresistance, where the MR has a maximum not for a perpendicular magnetic field, but when the angle between the current and magnetic field is $45^{\circ}$. The hole and electron density become similar around this angle, because of the Zeeman effect and create a maximum in the MR. This mechanism is supported by the angle-dependence and magnetic field-dependence of the carrier densities in the Drude model, as well as the observation of beating patterns in the $\mathrm{SdH}$ oscillations.

From careful analysis of the SdH-oscillations I can conclude that the tubular hole-like Fermi pocket in the $\Gamma Z$ direction of the $\mathrm{BZ}$ is a contributing factor to the butterfly MR, in combination with the larger, neighboring electron pocket. Rotating the sample in a magnetic field allows one to study the size of the extremal cross-section of the pocket as viewed from different angles. Any device that exhibits butterfly MR will show a transition from becoming larger with increasing angle to splitting into several distinct areas that become smaller with increasing angle. It remains uncertain, however, whether the topological properties of this hole-like Fermi pocket play a role in the creation of the butterfly MR or that the near-perfectly electron-hole compensated Drude model is solely responsible for this peculiar effect. By fitting a two-frequency Lifshitz-Kosevich model to the extracted Shubnikovde Haas oscillations and taking into account the appropriate phase shift for the Fermi pocket under study, I can conclude that the pocket has topological properties as it clearly shows a non-zero Berry phase that increases with angle. Although a field-dependence of the beating could not be confirmed, the Zeeman effect remains a likely candidate for the formation of the butterfly MR. 


\section{Appendix A: The Lifshitz-Kosevich model}

In 1954 Ilya Lifshitz and Arnold Kosevich found the connection between the Fermi surface and the oscillating magnetization of a material as a function of the applied field, known as the de Haas-van Alphen effect [187, 190, 191]. Oscillations in resistance, known as the Shubnikov-de Haas oscillations, have the same origin and can therefor also be described using the work of Lifshitz and Kosevich.

The model used in this chapter has four parameters that can be tuned to fit the model to the data. It is based on works in literature by $\mathrm{Hu}$ et al. [172] and Li et al. [189] and is written as a function of the magnetic field $B$ :

$$
\Delta R \propto B^{\lambda} R_{T} R_{D} R_{S} \sin \left[2 \pi\left(\frac{F}{B}+\phi\right)\right]
$$

where

$$
\begin{array}{r}
\phi=-\gamma-\delta \\
R_{T}=\frac{\alpha T \mu}{B} \sinh \left(\frac{\alpha T \mu}{B}\right) \\
R_{D}=e^{-\alpha T_{D} \mu / B} \\
R_{S}=\cos (\pi g \mu / 2)
\end{array}
$$

In these equations

$$
\begin{array}{r}
\alpha=\frac{2 \pi^{2} k_{B} m_{0}}{\hbar e} \\
\mu=m^{*} / m \\
\gamma=\frac{1}{2}-\phi_{B} / 2 \pi
\end{array}
$$

The frequency, $F$, Dingle temperature, $T_{D}$, phase of the signal, $\phi$, and a scaling constant, have been used as tunable parameters. Other parameters have been fixed. These are the factor $\lambda=1 / 2$, the ratio between the effective mass of the carriers and bare electron mass $\mu=0.08$, Landé factor $g=2$, and the measurement temperature, which was $T=2$ for all measurements. All other parameters, except for $\delta$ are physical constants. The parameter $\delta$ plays a crucial role in the extraction of the Berry phase from the total phase of the signal and was intensively studied by Li et al. as was mention in section 5.4 [189]. 



\section{Chapter}

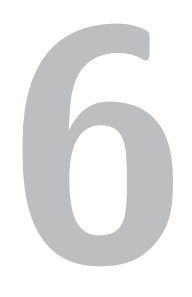

\section{S-wave superconductivity in $\mathrm{PdTe}_{2}$ observed by tunneling spectroscopy on side-junctions}

I have fabricated superconductor-normal metal side-junctions with different barrier transparencies out of $\mathrm{PdTe}_{2}$ crystalline flakes and measured the differential conductance spectra. Modeling the measurements using a modified Blonder Tinkham Klapwijk (BTK) formalism confirms that the superconductivity is mostly comprised of the conventional $s$-wave symmetry. It was found that for junctions with very low barrier transparencies, the junctions can enter a thermal regime, where the critical current becomes important. Adding this to the BTK-model allows us to accurately fit the experimental data. Finally I show additional features found in the $d I / d V$ spectrum of the fully ballistic junction, including a zero bias conductance peak. This peak cannot be adequately explained using only a conventional s-wave model and hints at a small contribution of helical $p$-wave pairing to the superconductivity.

This work was done in close collaboration with J.C. de Boer from the University of Twente. I have been primarily involved in device fabrication, measurements, and writing. 


\subsection{Introduction}

The search for the elusive Majorana particle has brought physicists to the area of topological superconductivity. The mixture of Dirac physics and superconductivity (SC) is seen as a promising way of creating Majorana quasiparticles [192], which in turn opens up the possibility of quantum computing through a process called braiding [49]. Experimental research has focused on the interface effects of superconductors coupled to either semiconductors with strong spin-orbit coupling [193, 194] or topological matter [195-198]. Topological superconductors, for example $\mathrm{Cu}_{x} \mathrm{Bi}_{2} \mathrm{Se}_{3}$, are also studied in the context of Majorana physics [199, 200]. The transition metal dichalcogenide $\mathrm{PdTe}_{2}$ belongs to the $\mathrm{P} \overline{3} \mathrm{~m} 1$ space group and is known to be a superconductor [119, 201, 202]. Recent experiments have shown that this material is also topological as it possesses a type-II Dirac cone [203-205], highlighting it as an extraordinary material, that could host unconventional superconductivity intrinsically [52]. Notably, Teknowijoyo et al. have narrowed the possible order parameter $(\mathrm{OP})$ symmetries down to three candidates: $\mathrm{A}_{1 g}$ (conventional $s$-wave) pairing, $\mathrm{A}_{1 u}$ (helical $p$-wave) pairing, or $\mathrm{E}_{u(1,0)}$ (nematic $p+f$-wave) pairing, by showing that the order parameter of $\mathrm{PdTe}_{2}$ is nodeless [120]. The latter two pairings are nontrivial. Experiments investigating the nature of the superconductivity in $\mathrm{PdTe}_{2}$ have so far found no indication of unconventional superconductivity [119].

In this chapter I present tunneling spectroscopy measurements performed on $\mathrm{PdTe}_{2}$-normal metal side-junctions, to shed light on the in-plane properties of the order parameter and distinguish between the three possible OPs that Teknowijoyo et al. have singled out. The data is modeled using a combination of the Blonder Tinkham Klapwijk (BTK) formalism [206] and the effect of the critical current, $I_{c}$, on the differential conductance [207$212]$. Finally, I show additional features found in the $d I / d V$ spectrum of the purely ballistic junction, together with an analysis of these features that are beyond our BTK and $I_{c}$-model.

\subsection{The experiment}

I have fabricated superconductor-(insulator-)normal metal ( $\mathrm{S}(\mathrm{I}) \mathrm{N})$ junctions out of exfoliated flakes of a $\mathrm{PdTe}_{2}$ crystal. The crystal has a preferred cleavage plane, which orientates all flakes with the $c$-axis out of plane. Y. Huang from the University of Amsterdam has grown a single crystal of $\mathrm{PdTe}_{2}$ by 
Chapter 6. S-wave superconductivity in $\mathrm{PdTe}_{2}$ observed by tunneling spectroscopy on side-junctions

a modified Bridgman method. High purity Pd (99.99\%) and Te (99.9999\%) were used as starting materials. The desired components were sealed in an evacuated cone-ended quartz ampoule. The ampoule was heated up to 800 ${ }^{\circ} \mathrm{C}$, kept for 48 hours and then cooled down to $500{ }^{\circ} \mathrm{C}$ at a rate of $3{ }^{\circ} \mathrm{C}$ per hour, followed by furnace cooling.

Section 2.3 gives a detailed description of the fabrication steps taken to create the devices on which the experiments in this chapter have been performed. For the sake of clarity, a small summary is included here as well. All devices are prepared by $\mathrm{Ar}^{+}$milling through the flake prior to the deposition of a barrier and normal metal, in order to create a side-contact, allowing us to probe the in-plane properties of the superconducting order parameter. All patterning for these steps was done using standard electronbeam lithography. The devices differ in their interfaces between the $\mathrm{PdTe}_{2}$ and the normal metal. The first type of devices was made without a specific barrier and is a $500 \mathrm{~nm}$ wide $\mathrm{SN}$ interface between $\mathrm{PdTe}_{2}$ and gold, with a normal state resistance $\left(R_{N}\right)$ of about $30 \Omega$ at $15 \mathrm{mK}$. The second type of devices was made by transferring the argon milled flakes to a sputter machine where they were cleaned of contaminations by low RF power plasma etching. On the cleaned surface, $1 \mathrm{~nm}$ of $\mathrm{Al}$ was sputter deposited, followed by oxidation in 10 mbar of oxygen for one hour to form an $\mathrm{Al}_{2} \mathrm{O}_{3}$ oxide barrier. To finalize the devices, a normal metal layer of palladium was sputter deposited on the aluminum oxide without breaking the vacuum. The $R_{N}$ was about $200 \Omega$ at $15 \mathrm{mK}$. The third type of devices was transferred to an atomic layer deposition (ALD) machine after argon milling, where a 1.2 $\mathrm{nm}$ thick $\mathrm{Al}_{2} \mathrm{O}_{3}$ layer was grown at $100{ }^{\circ} \mathrm{C}$, followed by ex-situ deposition of $40 \mathrm{~nm}$ of gold by sputter deposition. These SIN junctions have an $R_{N}$ of about $2 \mathrm{k} \Omega$ at $15 \mathrm{mK}$. For each type, measurements on one representative device are presented in this chapter.

A DC bias current with a small AC excitation is driven through the junctions while measuring both the $\mathrm{DC}$ and $\mathrm{AC}$ response across the junction to probe the differential resistance. The measured differential resistance is numerically inverted to differential conductance and plotted against the measured DC bias voltage. The results of these measurements at the lowest temperature reached $(T<100 \mathrm{mK})$ are shown as gray circles in figure 6.1. Comparing the three graphs we see a clear evolution of the main feature around zero bias. In figure 6.1(a) we see a dented plateau around zero bias, accompanied by sharp dips in conductance at $\pm 0.5 \mathrm{mV}$. Figure $6.1(\mathrm{~b})$ shows 

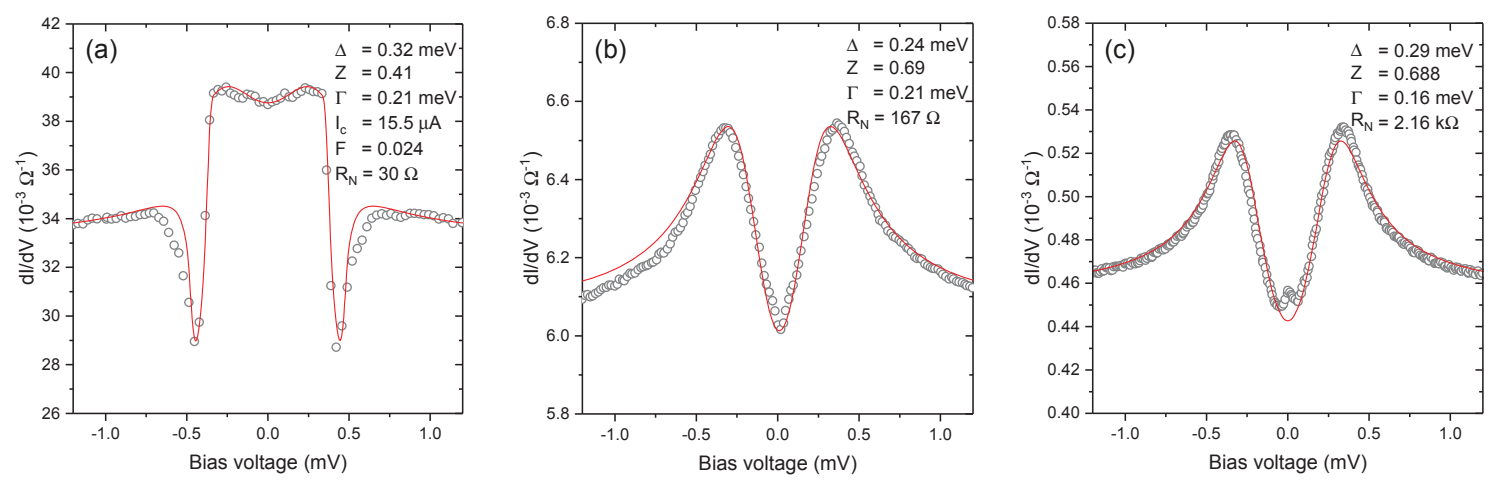

Figure 6.1: $d I / d V$ spectra of three $\mathrm{PdTe}_{2}$ junctions with different resistances measured at base temperature (gray circles). The red line is the best fit to the data. All relevant fitting parameters, as well as $R_{N}$, are included in the panel. (a) $d I / d V$ measurements and fit of a BTK-model with very transparent $\operatorname{PdTe}_{2} / \mathrm{Au}$ interface $\left(R_{N}=30 \Omega\right)$ and critical current effects. (b) $d I / d V$ measurements and fit of a BTK-model with slightly less transparent $\mathrm{PdTe}_{2} / \mathrm{Al}_{2} \mathrm{O}_{3} / \mathrm{Pd}$ interface $\left(R_{N}\right.$ $=167 \Omega$ ). (c) $d I / d V$ measurements and fit of a BTK-model with an opaque $\mathrm{PdTe}_{2} / \mathrm{Al}_{2} \mathrm{O}_{3} / \mathrm{Au}$ interface $\left(R_{n}=2.16 \mathrm{k} \Omega\right)$.

a quite different shape. The dented plateau around zero has been replaced by an Andreev-like spectrum with coherence peaks surrounding clear dip. The final device, of which the differential conductance is shown in figure 6.1(c), has the highest normal state resistance. Just as the data in figure 6.1(b), the measured $d I / d V$ spectrum in 6.1(c) looks like a clear Andreev spectrum. Around zero bias a small zero bias conductance peak (ZBCP) is visible.

\subsection{The BTK Model}

Because the $\mathrm{PdTe}_{2}$ flakes are less than $100 \mathrm{~nm}$ thick, which is less than the reported superconducting coherence length [120, 201], the conductance spectra are modeled numerically using a 2D BTK formalism for different order parameters. In this model, the bands are assumed to be parabolic since the Fermi energy is much larger than the energy where the type-II Dirac points reside [205]. The chemical potential mismatch, $\mu_{s c} / \mu_{n}$, is set to 1 for simplicity, so that $Z=H m_{e} / \hbar^{2} k_{s c}$, with $H$ the height of the $\delta$-shaped barrier, is the only barrier parameter. Teknowijoyo et al. have experimentally determined the $\mathrm{OP}$ in $\mathrm{PdTe}_{2}$ to be nodeless, which, together with crystal symmetry constraints, leaves us with three different pair potentials: $\mathrm{A}_{1 u}$ (conventional $s$-wave), $\mathrm{A}_{1 u}$ (helical $p$-wave), and $\mathrm{E}_{u(1,0)}$ (nematic $p+f$ - 
wave). The latter two correspond to the d-vectors $\mathbf{d}_{A_{1 u}}=k_{x} \hat{x}+k_{y} \hat{y}+k_{z} \hat{z}$ and $\mathbf{d}_{E_{u(1,0)}}=k_{x}\left(k_{x}^{2}-3 k_{y}^{2}\right) \hat{x}+k_{z} \hat{y}+k_{y} \hat{z}$. Before moving on to fitting the measured conductance spectra, let us look at the general features of the three OPs in a 2D BTK-model, where $k_{z}=0$. Figures 6.2(a)-(c) show the angle dependence of the superconducting gap magnitude. It is obvious from these plots that all three are nodeless. Note that the OPs of the $s$-wave, (a), and $\mathrm{A}_{1 u}$ pairing, (b), differ in their angle-dependence of the phase, rather than gap magnitude. Figures 6.2(d)-(f) show the normalized conductance as a function of the angle with respect to the interface normal. Brighter colors indicate a higher conductance in these graphs. Both the $\mathrm{A}_{1 u}$ and the $\mathrm{E}_{u(1,0)}$ pairing exhibit helical edge states within the superconducting gap. The panels labelled (g)-(i), show the calculated conductance spectra for dimensionless barrier strength $Z=0,0.5,1$, and 4 . The legend is included in figure (i). These $d I / d V$ spectra are the result of angle averaging over a semicircle directed at the interface.

Comparing the model to the differential conductance curves displayed in figure 6.1, it appears that only conventional $s$-wave pairing cannot adequately explain all our findings. Although the data obtained on the two high resistance devices can be nicely replicated using $s$-wave pairing with some degree of broadening, the sharp dips and elevated plateau of the lower resistance device are absent in figure $6.2(\mathrm{~g})$, which shows the resulting differential conductance of the $s$-wave BTK-model. The helical $p$-wave $d I / d V$, figure $6.2(\mathrm{~h})$, does exhibit sharp dips and a rising plateau, albeit far more rounded than the experimental data. Unconventional superconductivity, as long as it is nodeless [120], is not unimaginable in $\mathrm{PdTe}_{2}$ as the unique spin (or pseudo spin) structure of a Dirac semimetal (DSM) can stabilize unconventional pairing mechanisms $[52,200]$. However, using a combination of conventional $s$-wave and helical $p$-wave pairing, we were unable to accurately model the data of figures 6.1(a), i.e. the most transparent junction. The differential conductance of the two most resistive junctions, on the other hand, can be fitted well using only the conventional $s$-wave pairing model. The result of this fitting procedure is shown as a red line in figures 6.1(b) and (c). Every part, except for the ZBCP in 6.1(c) is described by the BTK-model, using a dimensionless barrier strength $Z \approx 0.7$. Let us leave the ZBCP for now and turn our attention to the lower $R_{N}$ junctions. 
(a)
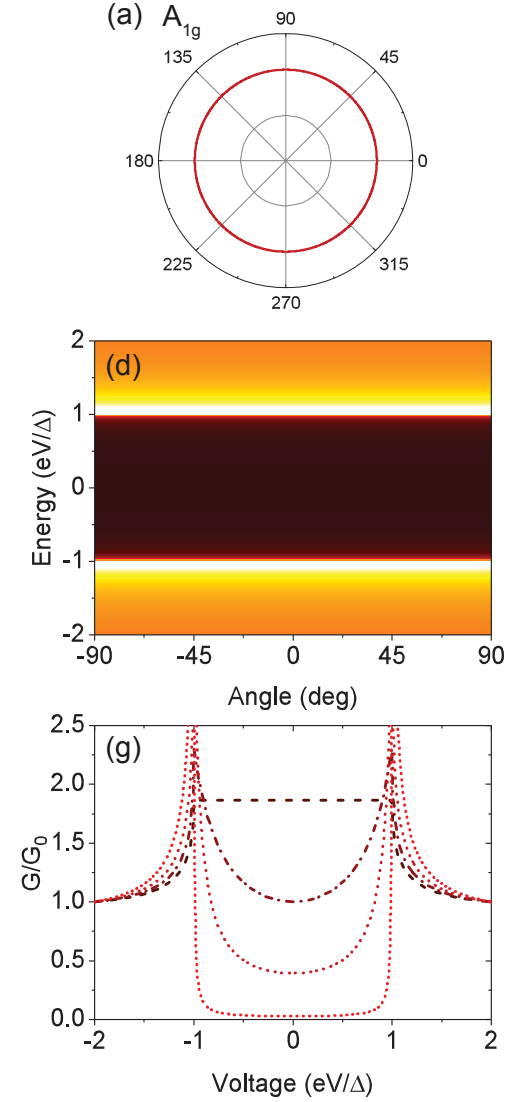

(b)
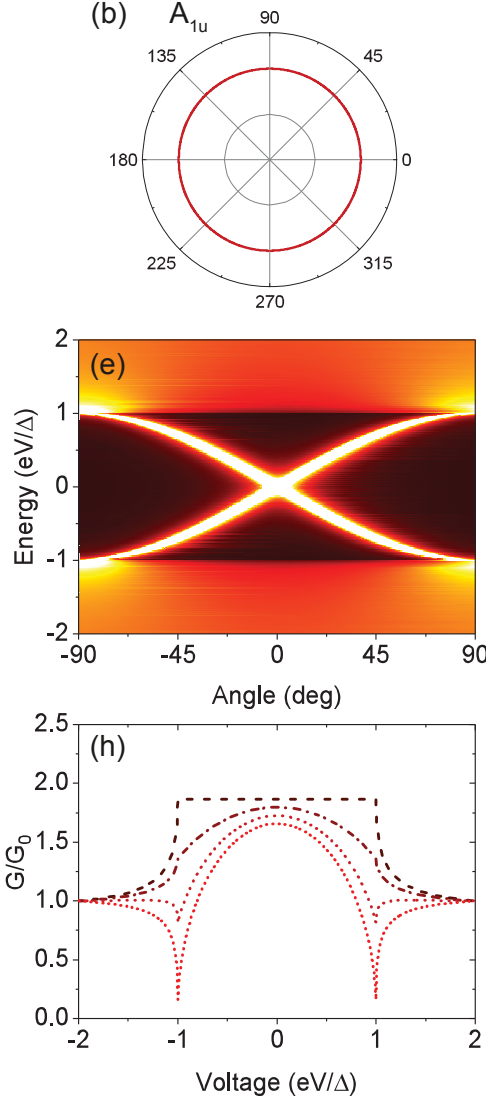

(c)
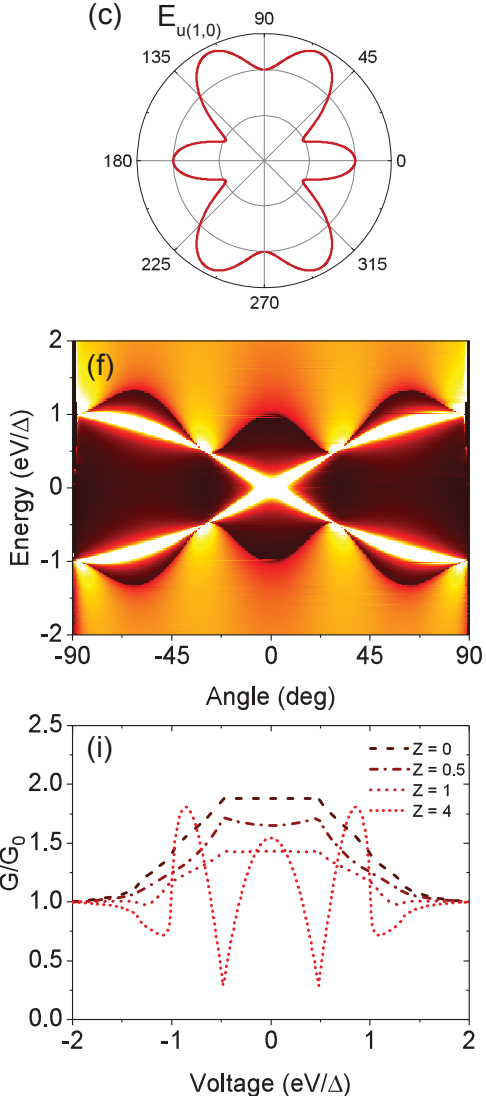

Figure 6.2: The BTK-model for conventional s-wave symmetry, $\mathrm{A}_{1 u}$, and $\mathrm{E}_{u(1,0)}$ pairing. (a)-(c) show the angle dependence of the gap, $\Delta$, indicating the shape of the OP and the fact that it is nodeless. (d)-(f) show the angle dependent conductance at different energies calculated for barrier strength $Z=4$. The color scale reflects the conductance, where brighter colors indicate higher conductance. Both $\mathrm{A}_{1 u}(\mathrm{e})$, and $\mathrm{E}_{u(1,0)}$, (f) have helical edge states at zero energy. Note that due to the anisotropy of the $\mathrm{E}_{u(1,0)}$ pair potential, some of the states with large $k_{y}$ components on the normal metal side have no superconducting equivalent at the same energy and result in zero conductivity. (g)-(i) are the conductance spectra obtained for different dimensionless barrier strengths, $Z$, in the BTK-model. They are the result of averaging the conductance over angles between -90 and +90 degrees. The legend in (i) shows which line represents which $Z$ and is valid for (g) and (h) as well. 


\subsection{The $\mathrm{I}_{c}+$ BTK Model}

To explain the origin of the sharp dips and dented plateau of figure 6.1(a) the BTK-model is extendedby taking into account the influence of the interfacial critical current on the obtained $d I / d V$ spectra [207-212]. The BTK-model assumes ballistic transport through the junction at all bias voltages, but in the case of very low resistance junctions, the junction may leave the ballistic regime and enter the thermal regime: while increasing the bias current through these transparent junctions we reach the critical current $I_{c}$, an effect that comes on top of the BTK-model. This critical current does not refer to the typical bulk $I_{c}$ of a superconductor, but rather to a reduced critical current in the disordered surface of the $\mathrm{PdTe}_{2}$ close to the interface. Upon reaching this critical current, a voltage suddenly appears across the junction, which is represented as a step in the IV-characteristic. Taking the derivative of this will yield sharp dips in $d I / d V$ at the critical current. Figure 6.3 schematically shows the device as it was modeled. It consists of three separate channels labelled in the schematic as "Diffusive", "Ballistic", and "Superconductor". The diffusive and ballistic channels are situated on the normal metal side of the interface and are treated as parallel conductance channels. The diffusive part is simply modeled as a resistor, which includes some Joule heating. The parallel ballistic channel is nothing more than the earlier described BTK model. Finally, the other side of the interface, labelled "Superconductor", is the disordered part of the $\mathrm{PdTe}_{2}$, where the critical current is lower than in the rest of the $\mathrm{PdTe}_{2}$. This conducting channel is included in series with the other two. The individual contributions of the three parts to the conductance are shown in figure 6.4, where the (a) panel shows the I-V characteristics and the (b) panel shows the differential conductance, which is the derivative of the curves in (a). The solid line of figure 6.4(b) has significant bearing on the interpretation of not only the data presented in this thesis, but of all conductance spectra that differ from the simple $s$-wave BTK shape. Although this curve is generated by a model that only incorporates conventional $s$-wave pairing, it looks like the textbook differential conductance spectrum of a $p$-wave superconductor. In fact, appendix A includes two identical looking fits of the differential conductance measured on a junction different from those previously reported in this chapter. One fit is made using the $\mathrm{I}_{c}+s$-wave BTK model, while the other is made using a helical $p$-wave BTK model. One should be aware that conventional superconductivity in some cases is easy to misinterpret as unconventional. Additional arguments should be provided on top of the agreement of the model with the data, to prove the existence of unconven- 


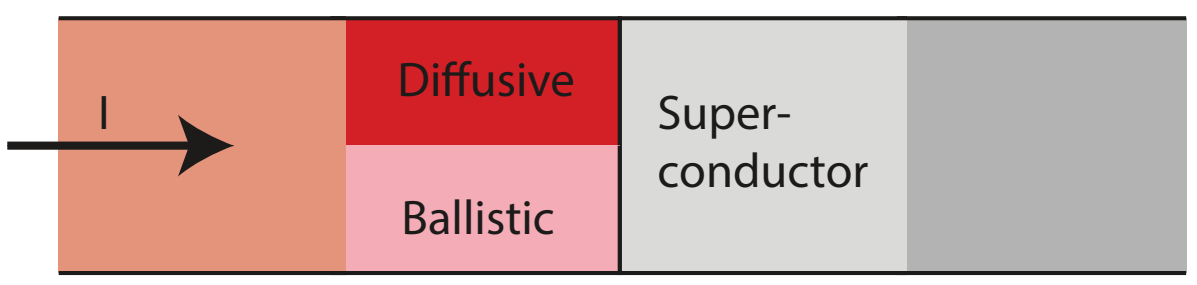

Figure 6.3: Schematic representation of the side-junction devices as they are modeled. The left side (red) represents the normal metal side of the junction. This side is modelled as a parallel diffusive channel and a ballistic channel (BTK). The right side (gray) represents the superconductor. The light-gray area near the SN interface is the disordered region, where the conductance spectrum is dominated by the local critical current.

tional superconductivity in one's device.

The red line in figures 6.1(a) shows the striking agreement of an $s$-wave BTK-model with our data, when the effect of $I_{c}$ is taken into account. The BTK parameters, as well as $I_{c}$, are reported inside the graph. $F$ describes the mixing of the $I_{c}$-model and the BTK-model through a linear combination, where $F=0$ means purely BTK-model and $F=1$ is purely the $I_{c}$-model. Note that this fitting is performed using only conventional $s$ wave pairing, like in figure 6.1(c). The device with the largest barrier is apparently in the ballistic regime for all applied currents, whereas this does not hold for the most transparent device. The $d I / d V$ features, occasionally ascribed to unconventional superconductivity, arise in our case from high transparency of the junction in combination with a disordered interface. This high transparency can be due to the design of the device, or an accidental feature, such as a pinhole or otherwise broken barrier.

\subsection{Additional features}

So far we have found that the main features of all three measured devices can be understood with the same OP symmetry, even though their differential conductance spectra differ greatly. The high resistance device is the best candidate for a more extensive conductance spectroscopy study, as it exhibits ballistic transport for up to $8 \mathrm{mV}$. So the $d I / d V$ spectrum of this device was measured at different temperatures and for a much larger range of bias currents. The experimental data is presented in figure 6.5(a). Figure 6.5(b) serves as a zoomed in version of this graph around zero bias and 
Chapter 6. S-wave superconductivity in $\mathrm{PdTe}_{2}$ observed by tunneling spectroscopy on side-junctions
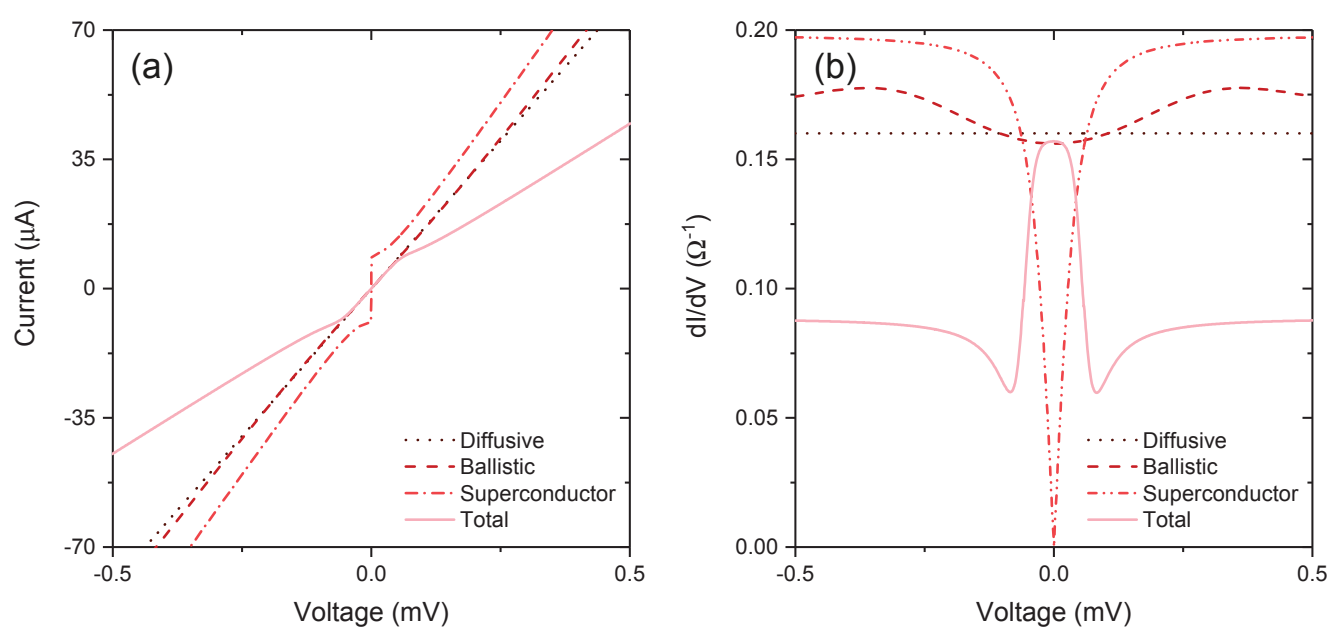

Figure 6.4: (a) $I-V$ characteristics of the separate parts of the interface, plotted against the voltage over this separate part. The total is modeled assuming a current bias setup. (b) The $d I / d V$ conductance spectra corresponding to the $I-V$ characteristics in panel a.

shows, as solid lines, the curves obtained from a conventional $s$-wave BTKmodel. The theoretically obtained curves describe the data near perfectly. A shallow dip-feature presents itself in figure 6.5(a) at bias voltages greater than $5 \mathrm{mV}$, far beyond the superconducting gap $(\sim 300 \mu \mathrm{V})$. The dip differs from the dips earlier attributed to the critical current. Such dips are quite sharp, since they relate to an instant increase in voltage, whereas this feature is shallow and stretched wide in voltage. Additionally, the position of this dip as a function of temperature is plotted in red circles in figure $6.5(\mathrm{c})$. They are accompanied by the superconducting gap $\Delta$ as extracted from the BTK fit on the low-bias part of this dataset. Both temperature dependences can be described using standard BCS theory. The two dashed lines show this standard BCS behavior, scaled to the voltage value at the lowest temperature. The dips generated by the $I_{c}$ do not necessarily follow the same temperature dependence as $\Delta$. Over the past decades there have been numerous experiments in which dips such as presented in figure have been observed [211-214]. The gradual loss and recurrence of conductance at high bias could be attributed to Andreev reflected holes recombining with quasiparticles inside the superconductor [215]. This removes carriers normally available for transport, thus decreasing the conductance. Another possible origin of this dip is that weak spots in the barrier are responsible for the crossover into the thermal regime at larger currents, similar to our $I_{C}$-model $[213,214]$. Although the exact mechanism that generates these features cannot be claimed definitively, we can examine one of the 

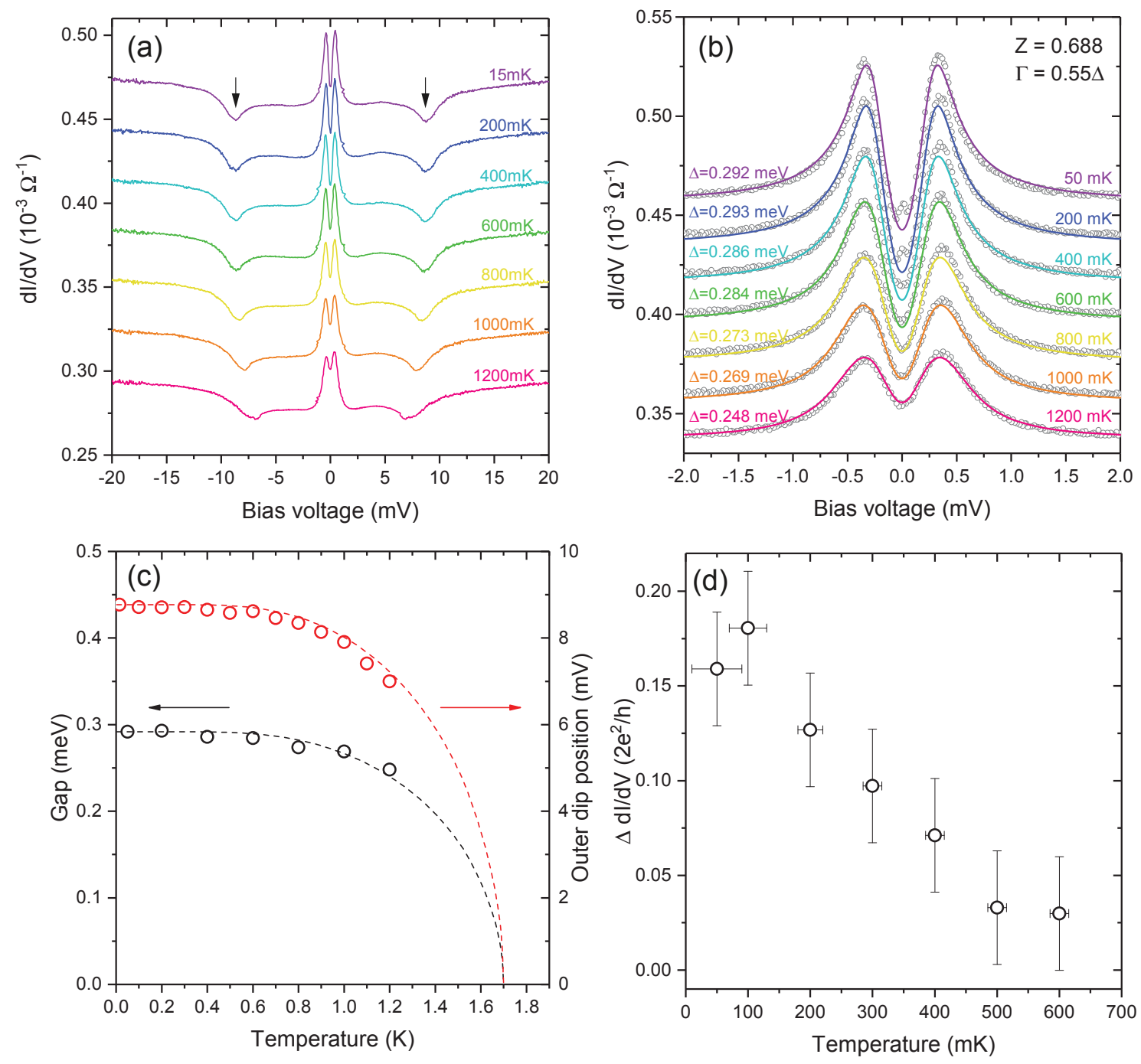

Figure 6.5: Additional measurements and analysis on the highest resistance sample. (a) $d I / d V$ of the $2.16 \mathrm{k} \Omega$ sample for different temperatures measured over a large range of bias voltage. For clarity, all the curves except for the $15 \mathrm{mK}$ curve have been given a constant offset. (b) $s$-wave BTK fits (colored lines) to the measured $d I / d V$ of the $2.16 \mathrm{k} \Omega$ junction (gray circles) at different temperatures. Again, all the curves except for the $15 \mathrm{mK}$ curve have been given a constant offset. The temperature and fitted gap $\Delta$ are indicated next to the line. $Z$ and $\Gamma$ are shared across the curves and are indicated in the top-right corner of the graph. (c) The superconducting gap from the BTK fits as a function of temperature (black circles) and the position of the shallow dip versus temperature (red circles). Dashed lines show the standard temperature dependence from BCS theory for a $T_{c}$ of $1.7 \mathrm{~K}$. (d) The height of the ZBCP as a function of temperature. 

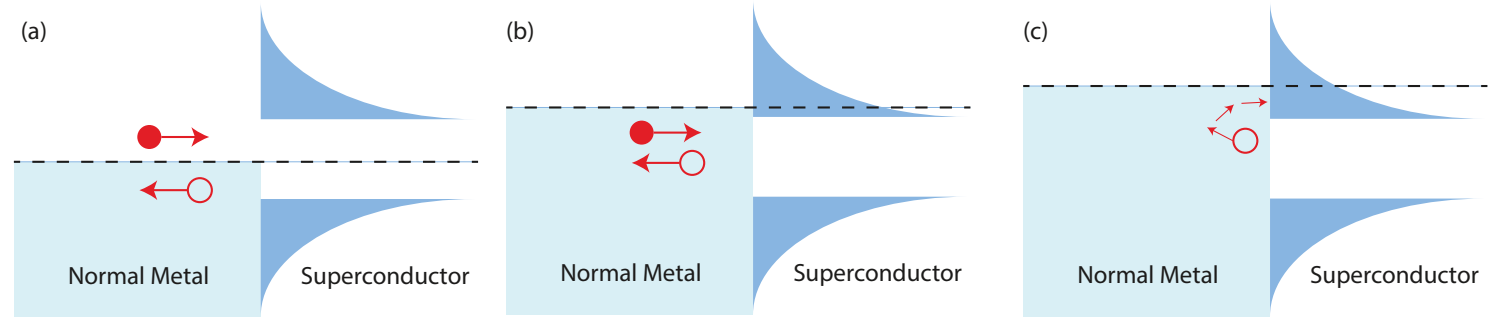

Figure 6.6: Processes at the NS interface for different bias voltages, $V$. The dashed line indicates the position of the Fermi level. Electrons and holes are represented by solid and open circles respectively.(a) At low bias voltages Andreev reflections occur at the NS interface. (b) When $V>\Delta$ Andreev reflections can still happen, albeit far more rarely. Because of the position of $E_{F}$ quasiparticles can also enter the superconductor. (c) An Andreev reflected hole enters the superconductor, where it can recombine with a quasiparticle. This prevents the AR hole from contributing to the conductance.

candidates in more detail. Besides the earlier mentioned $I_{c}$-effects there is the process wherein Andreev reflected holes recombine with quasiparticles inside the superconductor, leading to shallow and extended dips in the conductance. Figure 6.6 provides a visual aid to understanding this process. The recombination of $\mathrm{AR}$ holes inside the superconductor removes carriers normally available for transport, thus decreasing the conductance. For this effect to occur there must be Andreev reflected holes present, as well as quasiparticles to recombine with. Quasiparticle poisoning in the superconductor increases with bias voltage, but the Andreev reflected hole density decreases with increasing bias voltage. The convolution of these two effects has an optimum at a certain bias voltage, which leads to a shallow and extended dip in the differential conductance, such as shown in figure 6.5(a).

\section{The ZBCP}

The final, and perhaps most intriguing, feature is the aforementioned ZBCP. This peak can clearly be distinguished in low bias region of the low transparency device at sufficiently low temperatures. The BTK fits shown in figure $6.5(\mathrm{~b})$ are subtracted from the respective data allowing us to track the height of this peak as a function of temperature. The extracted peak heights are shown in figure 6.5(d). The height of the conductance peak is normalized to $2 e^{2} / h$, which is the value associated with tunneling into a Majorana mode [216]. Our ZBCP reaches about $20 \%$ of this value. The temperature dependence of the $\mathrm{ZBCP}$ is linear and the temperature at which the peak vanishes is much lower than the reported critical temperature of 


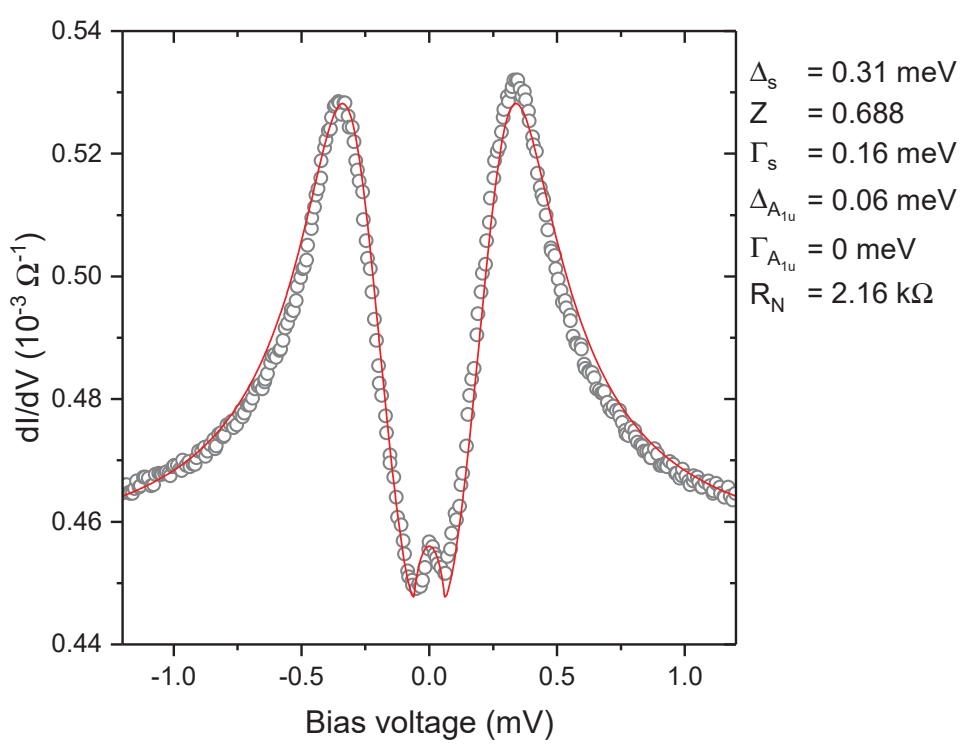

Figure 6.7: BTK fit (red line) to the experimentally obtained $d I / d V$ of the highest resistance sample at $50 \mathrm{mK}$ (gray circles). The model is a linear combination of $96 \%$ $s$-wave pairing and $4 \%$ helical $p$-wave pairing. The indicated fitting parameters, including the two gap magnitudes $\Delta$ are included in the top right corner of the panel.

$\mathrm{PdTe}_{2}$. At these temperatures the thermal energy, $k_{B} T$, is smaller than the width of the ZBCP, which hints to the fact that we are probing a different characteristic temperature here. It is important to note that a more intensive study is required to be able to make definite claims about the presence of unconventional superconductivity in $\mathrm{PdTe}_{2}$, since the $\mathrm{ZBCP}$ has only been observed in one device, albeit the most promosing device. I have not been able to fully describe the ZBCP by the $I_{c}+s$-wave BTK model. Once a small (4\%) $\mathrm{A}_{1 u}$ (helical $p$-wave) contribution is added to the $s$-wave BTK model, we obtain the red line shown in figure 6.7. The different contributions are modeled as two separate and parallel conduction channels. The obtained gap magnitude, $\Delta_{A_{1 u}}$ for the unconventional part relates to a $T_{c}$ of $400 \mathrm{mK}$ when I take $2 \Delta=3.52 k_{B} T_{c}$. Although this relation does not necessarily hold for unconventional superconductivity it is a fair estimate of the critical temperature of this phase and corresponds reasonably well with the experimental findings in figure $6.5(\mathrm{~d})$. It should be noted that no ZBCPs have been found in differential conductance measurements along the $c$-axis, which rules out $3 \mathrm{D}$ isotropic $\mathrm{A}_{1 u}$ pairing but leaves room for an anisotropic variant $[119,203]$. Recent studies of the superconductivity of $\mathrm{PdTe}_{2}$ have confirmed the existence of multiple superconducting channels, related to parallel bulk and surface superconductivity [201]. It is therefore 
Chapter 6. S-wave superconductivity in $\mathrm{PdTe}_{2}$ observed by tunneling spectroscopy on side-junctions

not unimaginable that the origin of the ZBCP lies in unconventional pairing in $\mathrm{PdTe}_{2}$, which results in topological surface states. If this is the case, the most likely pairing symmetry is $A_{1 u}$, which matches our analysis. The other nodeless pairing symmetry, $\mathrm{E}_{u(1,0)}$, consists of components that are linear in $k$ and cubic in $k$, i.e. $p$-wave $+f$-wave symmetry. This can behave like a fully gapped system only when the $k^{3}$ component is sufficiently strong compared to the linear term. Although the $\mathrm{E}_{u(1,0)}$ nematic $p+f$-wave state is unlikely to occur in nature, recent reports on the topological superconductor $\mathrm{Cu}_{x} \mathrm{Bi}_{2} \mathrm{Se}_{3}$ have found indications of $\mathrm{E}_{u}$ pairing symmetry [217-219].

In short, I have fabricated three $\mathrm{PdTe}_{2} /$ Normal metal side-junctions with different transparencies. Conductance spectroscopy measurements on the devices have shown that the order parameter has conventional $s$-wave symmetry. The shape of the conductance spectra heavily depends on the normal state resistance of the junction and can make conventional superconductivity look unconventional. One should exert caution in analyzing the data of low resistance SN junctions and confirm that the junction is solely in the ballistic limit, or include the effect of the critical current on the $d I / d V$ spectrum in one's model. The most opaque sample produced clear Andreev spectra in which a ZBCP has clearly been observed. Although I cannot claim the presence of unconventional superconductivity in $\mathrm{PdTe}_{2}$ based on a single device, the data shows indications of a small helical $p$-wave contribution. 
Appendix A: An example of a low resistance device that mimics p-wave behavior

\section{Appendix A. An example of a low resistance device that mimics $\mathrm{p}$-wave behavior}

One of the lowest resistance N(I)S devices fabricated for this work, has a resistance of $11.3 \Omega$. The measured conductance spectrum is shown in figure A.1 and exhibits a pronounced dome at zero bias, along with a parabolic background that is associated with Joule heating effects [208]. This spectrum perfectly matches what one would expect for a helical- $p$ superconductor with a high barrier (see Figure 2(h) of the main text).

Figure A.1(b) shows the excellent correspondence between the measured data and a numerical model for an $\mathrm{A}_{1 u}$ order parameter with $\Delta_{A_{1 u}}=0.085$ $\mathrm{meV}$, a dimensionless barrier strength $\mathrm{Z}=2.1$, and a small chemical potential mismatch $\mu_{s c} / \mu_{n}=1.15$. Despite the good fit to the data, the fitting parameters do not seem very appropriate for the device. Following the BCS model, the gap magnitude indicates $T_{c} \approx 560 \mathrm{mK}$, which does not match well to the temperature dependence in panel A.1(a). The dimensionless barrier strength of $\mathrm{Z}=2.1$ is very high for a device with a normal state resistance of $11.3 \Omega$. Contrary to the other datasets presented in the main text, interpreted as $p$-wave this spectrum shows no sign of an additional $s$-wave order parameter, which is another indication that these measurements should not be interpreted as fully described by the BTK formalism.

In figure A.1(c), the critical current model described in section A is fitted to the data. The fit parameters indicate $70 \%$ ballistic transport in the metal side of the barrier and a critical current $I_{c}=9.2 \mu \mathrm{A}$. Instead of fitting the BTK parameters to the data, we assumed the same values as found from fits in the main text because the influence of the $s$-wave BTK contribution is rather small. This $s$-wave $+I_{c}$ model matches just as well to the data as the $p$-wave model does, but in this case with much more realistic fit parameters.

The experimental data and theoretical fits presented in this section illustrate neatly the difficulties of studying the superconducting order parameter with a low resistance, point-contact like setup. To make hard statements about the nature of the superconducting order parameter, one preferably uses devices with thick barriers without pinholes, so that the resulting tunneling spectrum accurately corresponds to the density of states of the superconductor. 

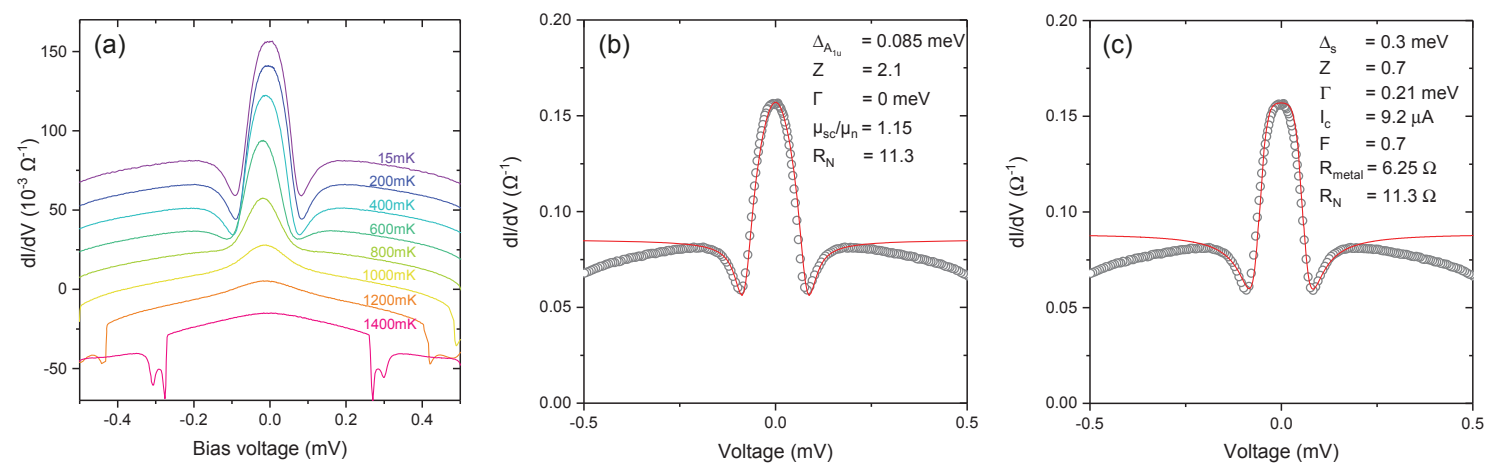

Figure A.1: (a) Temperature dependent $d I / d V$ spectra for an $R_{n}=11.3 \Omega \mathrm{N}(\mathrm{I}) \mathrm{S}$ device. The curves, except for $15 \mathrm{mK}$, are offset for clarity. (b) A 2D, $\mathrm{A}_{1 u}$ BTK model fitted to the $15 \mathrm{mK}$ data. (c) The $s$-wave $+I_{c}$ model, discussed in section A, fitted to the $15 \mathrm{mK}$ data. 


\section{Summary}

One of the most promising ways of creating computational devices beyond the limits of the current semiconductor technology, is being able to utilize the properties of the electron spin. This field of technology has been named spintronics. In this light, topological matter comes to mind since it has a fundamental property called spin-momentum locking. The fact that the spin orientation is directly coupled to the momentum direction of the charge carrier, could be the key to unlocking the field of spintronics. This thesis explores the role that the electron spin has on the electronic properties of a few recently discovered topological materials: $\mathrm{BiSbTeSe}_{2}$, a topological insulator designed to have very little contribution of the bulk to the electronic transport. Secondly, I study ZrSiS, a nodal-line semimetal that shows hints of a trivial to topological transition under a certain magnetic field. And finally, I investigate $\mathrm{PdTe}_{2}$, a material known to be both a superconductor and a Dirac semimetal, which makes it a good candidate for being a highly sought-after topological superconductor.

In chapter 3 the spin-momentum locking in $\mathrm{BiSbTeSe}_{2}$ is shown experimentally. To this end, I have placed topological insulator flakes on a strip of graphene, that can transport the spin-polarization because of its long spin-relaxation length. These devices allow for the creation of spinpolarization and its detection in a non-local configuration. By changing the magnetization of ferromagnetic leads with a magnetic field, the spinpolarization underneath the ferromagnetic lead was measured through the observed tunnel magnetoresistance. The experiments show that the spinpolarization changes linearly with the applied bias current, until increased scattering due to Joule heating diminishes the polarization. This experiment shows that spin-momentum locking indeed generates a discernible spin-polarization. Furthermore, the tuning of the Fermi level through backgating shows that the spin-polarization remains qualitatively unchanged when the carrier type is changed from electrons to holes.

Because of spin-momentum locking elastic scattering is suppressed in a topological insulator. The hyperfine interaction then plays a more important role in scattering processes. Effectively, this type of scattering transfers 
the electron spin to the bismuth nuclei. When the nuclear spins relax they again polarize the electrons, making the system act like a capacitor, or a battery. A square wave with a minimum voltage of zero is applied to the device while the voltage response of the device is measured. The extracted $\mathrm{RC}$-time is a measure for the capacitance that is present in the set-up. The experiments described in chapter 4 show no capacitance beyond the calibration, providing an upper limit to the performance of a topological insulator flake-based spin battery. In our measurements this upper limit was determined to be $0.1 \mathrm{nF}$.

Chapter 5 turns its attention to the nodal-line semimetal ZrSiS. Several research groups have reported on its curious angle-dependent magnetoresistance, called the butterfly magnetoresistance. By studying the magnetoresistance of several ZrSiS flakes from two different crystals, near-perfect electron hole compensation has been identified in this chapter as the origin of the butterfly magnetoresistance. For certain angles of the magnetic field the Zeeman shift slightly distorts the bands, which produces these equal, but opposite, carrier densities. In the two-band Drude model this leads to a large magnetoresistance. The beating pattern in the Shubnikov-de Haas oscillations supports this conclusion. Additionally, the Berry phase that is extracted from the Lifshitz-Kosevich fitting of quantum oscillations, increases with angle and hints towards a link between the butterfly magnetoresistance and the topological properties of $\mathrm{ZrSiS}$.

In the final chapter the order parameter symmetry of the superconductivity in $\mathrm{PdTe}_{2}$ is investigated by tunneling spectroscopy. Even though $\mathrm{PdTe}_{2}$ has been firmly established as a superconductor and a Dirac semimetal, it remains uncertain whether it also exhibits topological superconductivity. The experiments performed on Josepshon junctions in the ab-plane of $\mathrm{PdTe}_{2}$, combined with a theoretical model of the experiments, point to an important conclusion: in transparant Josephson junctions the conductance spectrum of conventional superconductivity can be nearly indistinguishable from unconventional superconductivity. The addition of effects related to the critical current in the disordered region near the interface to the model, is required to properly interpret the conductance spectra. In the conductance spectrum obtained from most opaque junction, there is a small feature that could not be explained without the addition of $4 \%$ helical $p$-wave symmetry to the model. This hints to the presence of unconventional superconductivity in $\mathrm{PdTe}_{2}$. 



\section{Samenvatting}

Een van de meest veelbelovende manieren om computerelementen te creëren die voorbijgaan aan de huidige limieten van de halfgeleidertechnologie, is om gebruik te maken van de spin van een elektron. Deze technologie wordt spintronica genoemd. Met deze gedachte in het achterhoofd, kom je al snel uit op topologische materie, omdat het de fundamentele eigenschap heeft genaamd spin-impulskoppeling. Deze koppeling van de spinoriëntatie aan de impulsrichting kan de sleutel zijn tot het ontwikkelen van spintronica. In dit proefschrift wordt onderzocht welke rol de elektron spin speelt in de elektronische eigenschappen van een aantal recentelijk ontdekte topologische materialen. Ten eerste, BiSbTeSe 2 , een topologische isolator die zo is ontworpen dat het een zo klein mogelijke bijdrage van de bulk toestanden heeft aan de transporteigenschappen. Daarnaast bestudeer ik ZrSiS, een halfmetaal met een ring van Dirac punten, dat een overgang lijkt te ondergaan van een triviale naar een topologische toestand als gevolg van een bepaald magneetveld. En tot slot onderzoek ik $\mathrm{PdTe}_{2}$, een materiaal dat zowel bekend staat als supergeleider als als Dirac halfmetaal, wat het tot een goede kandidaat maakt om een felbegeerde topologische supergeleider te zijn.

In hoofdstuk 3 wordt de spin-impulskoppeling in $\mathrm{BiSbTeSe}_{2}$ experimenteel aangetoond. Om dit te doen, heb ik vlokken van deze topologische isolator geplaatst op grafeen, dat vanwege de lange spinrelaxatielengte de spinpolarisatie kan doorgeven zonder het te verliezen. Deze structuren kunnen een spin-polarisatie generen, doorgeven, en detecteren in een niet-lokale opstelling. Door de magnetisatie van een ferromagnetisch contact te veranderen door middel van een magneetveld kan de spinpolarisatie onder dit contact bepaald worden door de tunnelmagnetoweerstand te bekijken. Uit deze experimenten blijkt dat de spinpolarisatie lineair verandert met de aangelegde stroom, totdat de verstrooiing door Joule verhitting deze spinpolarisatie weer verkleint. Het experiment toont aan dat de spin-impulskoppeling inderdaad een meetbare spinpolarisatie kan generen. Daarnaast kan het Fermi niveau verschoven worden door middel van het veldeffect. Hiermee is laten zien dat de spinpolarisatie niet verandert als het ladingsdragertype 
van elektronen in gaten wordt veranderd.

Omdat spin-impulskoppeling de elastische verstrooiing in een topologische isolator onderdrukt, speelt de hyperfijne interactie een belangrijkere rol in de verstrooiingsprocessen. Dit komt neer op het feit dat wanneer een elektron wordt verstrooid het zijn spininformatie doorgeeft aan een bismutkern. Als de kernspin van de bismutkernen weer terug valt, polariseert dit weer elektronen, waardoor het systeem zich in feite gedraagt als een condensator, ofwel een spin batterij. Een blokgolf met een minimum van nul wordt aangelegd over het experiment terwijl het voltageverschil over de structuur wordt gemeten. De RC-tijd die hieruit gehaald wordt, is een maat voor de capaciteit dit aanwezig is in de meetopstelling. De experimenten die beschreven zijn in hoofdstuk 4 laten geen capaciteit zien bovenop de gemaakte kalibratie, hetgeen een limiet van $0.1 \mathrm{nF}$ stelt aan de prestatie van een spinbatterij die gebaseerd is op topologische isolatorkristallen.

Hoofdstuk 5 richt de aandacht op het halfmetaal ZrSiS. Een aantal onderzoeksgroepen heeft al verslag gedaan van de interessante hoekafhankelijke magnetoweerstand van dit materiaal, genaamd de vlindermagnetoweerstand. Door de magnetoweerstand van twee verschillende kristallen te bestuderen, is de nagenoeg perfecte electron-gat verhouding in dit hoofdstuk aangewezen als oorzaak van de vlindermagnetoweerstand. Bij sommige hoeken van het magneetveld kan het Zeeman effect de banden een beetje verbuigen, waardoor er gelijke, maar tegengestelde, ladingsdragerdichtheden onstaan. In het tweebands Drudemodel leidt dit tot een grote magnetoweerstand. Het zwevingspatroon van de Shubnikov-de Haas oscillaties ondersteunt deze conclusie. Daarnaast duidt de Berryfase, die is gehaald uit het toepassen van het Lifshitz-Kosevich model op de kwantumoscillaties, er op dat een verband is tussen de vlindermagnetoweerstand en de topologische eigenschappen van $\mathrm{ZrSiS}$.

In het laatste hoofdstuk wordt de ordeparametersymmetrie van de supergeleiding in $\mathrm{PdTe}_{2}$ onderzocht door middel van tunnelspectroscopie. Het staat vast dat $\mathrm{PdTe}_{2}$ zowel een supergeleider als een Dirac halfmetaal is, maar het is vooralsnog niet zeker of het ook topologische supergeleiding vertoont. De experimenten die in dit hoofdstuk worden verricht aan Josephson juncties in het ab-vlak van $\mathrm{PdTe}_{2}$, wijzen op een belangrijke conclusie: in transparante Josephson juncties is het geleidinsspectrum van conventionele supergeleiding vrijwel identiek aan dat van onconventionele supergeleiding. 
Het is noodzakelijk om effecten, die te maken hebben met de kritische stroom in het ongeordende gebied aan het grensvlak, mee te nemen in het model om de geleidingsspectra correct te interpreteren. In de geleidingsspectra gemeten aan de minst transparante junctie is een kleine afwijking te zien, die ik niet kan verklaren zonder $4 \%$ schroefvormige $p$-golf symmetrie aan het model toe te voegen. Dit is een aanwijzing dat er onconventionele supergeleiding aanwezig kan zijn in $\mathrm{PdTe}_{2}$. 



\section{Dankwoord}

Tien jaar geleden kwam ik naar Enschede om te beginnen aan mijn studie. Ik had niet kunnen vermoeden dat ik nu, nog altijd in Enschede, de laatste hand aan mijn proefschrift zou leggen. Mijn tijd in Enschede is kleur gegeven door de vele kleurrijke mensen om mij heen.

Allereerst wil ik Alexander bedanken, die mij inspireerde en motiveerde om te gaan beginnen aan dit vierjarige avontuur. Juist het fenomeen van papadagen illustreert waarom promoveren bij jou zo prettig is: de diepgravende natuurkunde wordt gecombineerd met een onmisbaar stukje menselijkheid.

李川,谢谢你成为我的老师. Without you, my work in the lab and in the office would have never reached this level. You never stop helping people, even when it has nothing to do with science. Thank you for being a constant motivation to learn more. And sorry for all the shitty music.

Zonder Dick en Frank, geen ICE/QTM. Jullie voorkomen niet alleen dat onze labs vervallen tot een hoopje RVS, maar waken ook voor het sociale verval van de groep. Wanneer Dick, met zijn lepeltje in zijn mok tikkend als een cipiers wapenstok tussen de tralies, langs loopt in de ochtend weet je het al: koffietijd! Alle lof en dank voor het werk dat jullie doen, waardoor ook de rest van de groep iets gedaan krijgt.

Mijn tijd als PhD had de meeste kleur in de perioden dat ik mijn tijd mocht delen met anderen. Daan, Luca, Martina, Liesbeth en iedereen die ooit bij me langs is gekomen met een vraag. Bedankt voor het vertrouwen dat jullie in mij hadden en de gezelligheid die jullie brachten. Ik ben erg blij, en stiekem ook een beetje trots, dat jullie nu nog altijd bij ICE/QTM (of PIN) te vinden zijn.

Bob, jou ben ik misschien meer excuses dan dank verschuldigd. Vier jaar lang heb je mijn muziek moeten aanhoren en heb ik de verwarming hoger gezet. Bedankt voor het bijna vijf jaar samenwerken, je adviezen, je samples, 
het ijken van mijn horloge op 9:45 en natuurlijk de vele gesprekken over volleybal!

En dan mijn partner-in-crime, Jorrit. Als ik de baas was van de NPO, kreeg jij je eigen voetbalpraatprogramma. Of beter, ik zou Pierre van Hooijdonk overal vervangen door jou. Ik wil je niet alleen bedanken voor het oeverloze gelul over voetbal, FIFA, voetbalnieuws, natuurkunde, politiek, en voetbal, maar ook voor de geweldige samenwerking. Een dag werk mét of zónder Jorrit is een groot verschil. Zelfs op 1e kerstdag helium vullen, wordt met jou een feestje.

Uiteraard wil ik iedereen van ICE/QTM van harte bedanken. De mensen maken de groep. Zonder koffie, fruit, taart, gepraat aan de koffietafel, VriMiBo's, sportdagen, DASH, colloquia, ICE-ijs, skitripjes en schaatsuitjes, is een PhD-opdracht niet hetzelfde. Hans, Ans, Jaap, Sasha, Omar, Sander, Pim, Martijn, Denise, Ankur, Gerben, Gerben, Sebastian, en vele anderen die ik heb mogen leren kennen in mijn tijd bij ICE/QTM: dank!

De afgelopen vier jaar zijn door vele volleyballers voorzien van een beetje extra oranje en paars. Of in het geval van de U66R, een beetje extra rood. Синергия принадлежит всем нам! Naast het zelf volleyballen, heb ik ook het meest geweldige team van Harambee drie jaar lang mogen begeleiden. Ik wil alle dames van Dames 9 bedanken voor hun inzet en vuur, zowel binnen als buiten het veld. Het is geweldig om mijn liefde voor volleybal te mogen delen met zulke enthousiaste mensen.

In het bijzonder wil ik Ronald bedanken. Je eeuwige rust in alles dat je doet is een bron van inspiratie, die ik onvoldoende benut. Bedankt ook voor het delen van mijn passie voor spelletjes. Zonder jou stonden er kilo's karton doelloos mijn kast.

De combinatie volleybal en spelletjes is een gouden combinatie gebleken over de afgelopen jaren. Roy, Niels, Axel, Gerrit, Sander, Yuri, Nadia, en Tim, ook al volleybal je niet, bedankt!

Mijn liefde voor leren in het algemeen en de natuurkunde in het bijzonder heb ik te danken aan mijn ouders. Pap en mam, het is dankzij jullie onvoorwaardelijke steun in alle tijden dat ik nu deze laatste paar regels kan 
toevoegen aan mijn proefschrift. Het is mij ontzettend waardevol dat ik nu mijn promotie met jullie kan delen.

Tot slot wil ik mijn bron van liefde en steun, Sanne, bedanken. Je motiveert me om mezelf te blijven verbeteren en daarvoor ben ik je eeuwig dankbaar. Je hebt mijn leven alle mogelijke kleuren gegeven in de afgelopen jaren. Hoewel mijn boekje hier eindigt, is het onze pas net begonnen. 



\section{Bibliography}

[1] The Nobel prize in Physics 1956, https://www.nobelprize.org/ prizes/physics/1956/summary/ Accessed 1-Nov-2018

[2] Intel $14 \mathrm{~nm}$ technology, https://www.intel.com/content/www/us/ en/silicon-innovations/intel-14nm-technology.html Accessed $1-N o v-2018$

[3] H. Iwai, Micr. Eng. 86, 1520-1528 (2009)

[4] The international technology roadmap for semiconductors published by the ITRS (2015), https://www. semiconductors . org/wp-content/uploads/2018/06/0_2015-ITRS-2 . 0-Executive-Report-1.pdf, Accessed 1-Nov-2018

[5] V.K. Joshi, Eng. Sci. and Tech. 19, 1503-1513 (2016)

[6] E. Pop, S. Sinha, and K.E. Goodson, Proc. IEEE 98, 8, 1587-1601 (2006)

[7] A. Hoffman and S.D. Bader, Phys. Rev. App. 4, 047001 (2015)

[8] I. Zutic, J. Fabian, and S. Das Sarma, Rev. Mod. Phys. 76, 323-410 (2004)

[9] David. J. Griffiths, Introduction to Quantum Mechanics, Second edition (2005), ISBN 0131911759

[10] N.W. Ashcroft and N.D. Mermin, Solid state physics, ISBN-13: 9780-09-083993-1

[11] B. Yan, and C. Felser, Ann. Rev. of Cond. Mat. Phys., 8 337-354 (2017)

[12] N.P. Armitage, E.J. Mele, and A. Vishwanath, Rev. Mod. Phys., 90, 015001 
[13] H. Weng, C. Fang, Z. Fang, B.A. Bernevig, and X. Dai, Phys. Rev. $\mathrm{X}, \mathbf{5}, 011029(2015)$

[14] S. Huang, S. Xu, I. Belopolski, C. Lee, G. Chang, B. Wang, N. Alidoust, G. Bian, M. Neupane, C. Zhang, S. Jia, A. Bansil, H. Lin, and M.Z. Hasan, Nat. Comm., 6, 7373 (2015)

[15] S. Xu, I. Belopolski, N. Alidoust, M. Neupane, G. Bian, C. Zhang, R. Sankar, G. Chang, Z. Yuan, C. Lee, S. Huang, H. Zheng, D.S. Sanchez, B. Wang, A. Bansil, F. Chou, P.P. Shibayev, H. Lin, S. Jia, and M.Z. Hasan, Science, 349, 6248, 613-617 (2015)

[16] L. Lu, Z. Wang, L. Ran, L. Fu, J.D. Joannopoulos, and M. Soljačic, Science, 349, 6248 622-624 (2015)

[17] H. Weyl, Z. Phys. A: Hadrons Nucl., 56, 330-352 (1929)

[18] Z. Li, H. Chen, S. Jin, D. Gan, W. Wang, L. Guo, and X. Chen, Cryst. Growth Des., 2016, 16 1172-1175 (2016)

[19] G. Montambaux, F. Piéchon, J.-N. Fuchs, and M.O. Goerbig, Phys. Rev. B, 80, 153412 (2009)

[20] Z. Wang, Y. Sun, X. Chen, C. Franchini, G. Xu, H. Weng, X. Dai, and Z. Fang, Phys. Rev. B, 85, 195320 (2012)

[21] S.M. Young, S. Zaheer, J.C.Y. Teo, C.L. Kane, E.J. Mele, and A.M. Rappe, Phys. Rev. Lett., 108, 140405 (2012)

[22] M. Neupane, S. Xu, R. Sankar, N. Alidoust, G. Bian, C. Liu, I. Belopolski, T. Chang, H. Jeng, H. Lin, A. Bansil, F. Chou, and M.Z. Hasan, Nat. Comm., 5, 3786 (2014)

[23] Z.K. Liu, B. Zhou, Y. Zhang, Z.J. Wang, H.M. Weng, D. Prabhakaran, S.-K. Mo, Z.X. Shen, Z. Fang, X. Dai, Z. Hussain, and Y.L. Chen, Science, 343, 864-867 (2014)

[24] H.J. Kim, K.S. Kim, J.F. Wang, M. Sasaki, N. Satoh, A. Ohnishi, M. Kitaura, M. Yang, and L. Li, Phys. Rev. Lett. 111, 246603 (2013)

[25] M. Neupane, I. Belopolski, M. Mofazzel Hosen, D.S. Sanchez, R. Sankar, M. Szlawska, S. Xu, K. Dimitri, N. Dhakal, P. Maldonado, P.M. Oppeneer, D. Kaczorowski, F. Chou, M.Zahid Hasan, and T. Durakiewicz, Phys. Rev. B, 93, 201104 (2016) 
[26] E.H. Hall, Am. J. of Math. 2, 3 287-292 (1879)

[27] E.H. Hall, Philos. Mag. 12, 157 (1981)

[28] R. Karplus and J.M. Luttinger, Phys. Rev., 95, 5 (1954)

[29] N. Nagaosa, J. Sinova, S. Onoda, A.H. MacDonald and N.P. Ong, Rev. Mod. Phys., 82, 1539 (2010)

[30] K. von Klitzing, G. Dorda, and M. Pepper, Phys. Rev. Lett., 45, 7 (1980)

[31] The Nobel prize in Physics 1985, https://www.nobelprize.org/ prizes/physics/1985/summary/ Accessed 2-Nov-2018

[32] X-L. Qi and S-C. Zhang, Phys. Today, 63, 1, 33 (2010)

[33] J.E. Moore Nature, 464, 194-198 (2010)

[34] M.Z. Hasan and C.L. Kane, Rev. Mod. Phys. 82, 3045 (2010)

[35] Y. Ando, J. Phys. Soc. Jpn. 82, 102011 (2013)

[36] N. Tombros, C. Jozsa, M. Popincuic, H.T. Jonkman, and B.J. van Wees, Nature, 2007, 448, 571-574 (2007)

[37] W. Han, K.M. McCreary, K. Pi, W.H. Wang, Y. Li, H. Wen, J.R. Chen, R.K. Kawakami, J. Magn. and Magn. Mat., 324, 369-381 (2012)

[38] V.V. Schmidt, The physics of superconductors - Introduction to fundamentals and applications, 1997, ISBN 3-540-61243-2

[39] J. Bardeen, L.N. Cooper, and J.R. Schrieffer, Phys. Rev., 108, 5 1175$1204(1957)$

[40] M.R. Norman, Science, 332, 196-200 (2011)

[41] M. Sigrist, and K. Ueda, Rev. Mod. Phys., 62, 2 239-311 (1991)

[42] K. Ishida, H. Mukuda, Y. Kitaoka, K. Asayama, Z.Q. Mao, Y. Mori, and Y. Maeno, Nature, 396, 658-660

[43] A.P. Mackenzie, and Y. Maeno, Rev. Mod. Phys., 75, 657-712 
[44] D.A. Wollman, D.J. Van Harlingen, W. C.Lee, D.M. Ginsberg, and A.J. Leggett, Phys. Rev. Lett., 71, 2134 (1993)

[45] A.F. Andreev, Sov. Phys. JETP, 19, 1228

[46] C.W.J. Beenakker, Rev. Mod. Phys., 80, 4 1337-1354 (2008)

[47] E. Majorana, Il Nuovo Cimento, 14, 171-184 (1937)

[48] L. Fu, and C.L. Kane, Phys. Rev. Lett., 100096407

[49] S. Das Sarma, M. Freedman, and C.Nayak, npj Quantum Information, 1, 15001

[50] X-L. Qi and S-C. Zhang, Rev. Mod. Phys. 83, 1057 (2011)

[51] L. Fu, C.L. Kane, and E.J. Mele, Phys. Rev. Lett. 98, 106803 (2007)

[52] M. Sato, and Y. Ando, Rep. Prog. Phys., 80, 076501 (2017)

[53] M. Julliere, Phys. Lett. A, 54, 2, 225-226 (1975)

[54] M.N. Baibich, J.M. Broto, A. Fert, F.N. van Dau, and F. Petroff, Phys. Rev. Lett., 61, 21 2472-2475 (1988)

[55] G. Binasch, P. Grünberg, F. Saurenbach, and W. Zinn, Phys. Rev. B, 39, 7 4828-4830 (1989)

[56] The Nobel prize in Physics 2007, https://www.nobelprize.org/ prizes/physics/2007/summary/ Accessed 5-Nov-2018

[57] X. Fong, Y. Kim, R. Venkatesan, S.H. Choday, A. Raghunathan, and K. Roy, Proc. of the IEEE, 104, 7 1449-1488 (2016)

[58] L. Liu, O.J. Lee, T.J. Gudmundsen, D.C. Ralph, and R.A. Buhrman, Phys. Rev. Lett., 109, 096602 (2012)

[59] I.M. Miron, G. Gaudin, S. Auffret, B. Rodmacq, A. Schuhl, S. Pizzini, J. Vogel, and P. Gambardella, Nat. Mat., 9, 230-234 (2010)

[60] M.I. Dyakonov, and V.I. Perel, Sov. Phys. JEPT Lett., 13, 467 (1971)

[61] M.I. Dyakonov, and V.I. Perel, Phys. Lett. A, 35, 6, 459-460 (1971)

[62] Y.K. Kato, R.C. Meyers, A.C. Gossard, and D.D. Awschalom, Science, 306, 1910 (2004) 
[63] J. Wunderlich, B. Kaestner, J. Sinova, and T. Jungwirth, Phys. Rev. Lett., 94, 047204 (2005)

[64] Y. Ando, and M. Shiraishi, J. Phys. Soc. Jpn., 86, 011011 (2017)

[65] A. Manchon, H.C. Koo, J. Nitta, S.M. Frolov, and R.A. Duine, Nat. Mat., 14, 871-882 (2015)

[66] J. Puebla, F. Auvray, M. Xu, B. Rana, A. Albouy, H. Tsai, K. Kondou, G. Tatara, and Y. Otani, Appl. Phys. Lett., 111, 092402 (2017)

[67] A.R. Mellnik, J.S. Lee, A. Richardella, J.L. Grap, P.J. Mintun, M.H. Fischer, A. Vaezi, A. Manchon, E.-A. Kim, N. Samarth, and D.C. Ralph, Nature, 511, 449-451 (2014)

[68] Y. Fan, P. Upadhyaya, X. Kou, M. Lang, S. Takei, Z. Wang, J. Tang, L. He, L-T. Chang, M. Montazeri, G. Yu, W. Jiang, T. Nie, R.N. Schwarts, Y. Tserkovnyak, and K.L. Wang, Nat. Mat., 13, 699704 (2014)

[69] K.S. Novoselov, A.K. Geim, S.V. Morozov, D. Jiang, Y. Zhang, S.V. Dubonos, I.V. Grigorieva, and A.A. Firsov, Science, 306, 666-669 (2004)

[70] W. Han, R.K. Kawakami, M. Gmitra, and J. Fabian, Nat. Nanotech., 9, 794-807 (2014)

[71] D. Pesin, and A.H. MacDonald, Nat. Mat., 11, 409-416 (2013)

[72] D. Huertas-Hernando, F. Guinea, and A. Brataas, Phys. Rev. Lett., 103, 146801 (2009)

[73] M.S.A. Bhuyan, M.N. Uddin, M.M. Islam, F.A. Bipasha, and S.S. Hossaim, Int. Nano Lett., 6, 65-83 (2016)

[74] W. Han, K. Pi, W. Bao, M. McCreary, Y. Li, W.H. Wang, C.N. Lau, and R.K. Kawakami, Appl. Phys. Lett., 94, 222109 (2009)

[75] C. Józsa, T. Maassen, M. Popinciuc, P.J. Zomer, A. Veligura, H.T. Jonkman, and B.J. van Wees, Phys. Rev. B, 80, 241403(R) (2009)

[76] X. Li, W. Cai, J. An, S. Kim, J. Nah, D. Yang, R. Piner, A. Velamakanni, I. Jung, E. Tutoc, S.K. Banerjee, L. Colombo, and R.S. Ruoff, Science, 324, 1312-1314 (2009) 
[77] M.V. Kamalakar, C. Groenveld, A. Dankert, and S.P. Dash, Nat. Comm., 6, 6766 (2015)

[78] H. Cao, Q. Yu, L.A. Jauregui, J. Tian, W. Wu, Z. Liu, R. Jalilian, D.K. Benjamin, Z. Jiang, J. Bao, S.S. Pei, and Y.P. Chen, Appl. Phys. Lett., 96, 122106 (2010)

[79] J. Balakrishnan, G.K.W. Koon, A. Avsar, Y. Ho, J.H. Lee, M. Jaiswal, S.-J. Baeck, J.-H. Ahn, A. Ferreira, M.A. Cazalilla, A.H. Castro Neto, and B. Özyilmaz, Nat. Comm. 5, 4748 (2014)

[80] A.H. Castro Neto, and F. Guinea, Phys. Rev. Lett., 103, 026804 (2009)

[81] A.K. Patra, S. Singh, B. Barin, Y. Lee, J-H. Ahn, E. del Barco, E.R. Mucciolo, and B. Özyilmaz, Appl. Phys. Lett., 101, 162407 (2012)

[82] J.W. Suk, A. Kitt, C.W. Magnunson, Y. Hao, S. Ahmed, J. An, A.K. Swan, B.B. Goldberg, and R.S. Ruoff, ACS Nano, 5, 6916-6924 (2011)

[83] N. Petrone, C.R. Dean, I. Meric, A.M. van der Zande, P.Y. Huang, L. Wang, D. Muller, K.L. Shepard, and J. Hone, Nano Lett., 12, $2751-2756(2012)$

[84] O.A. Pankratov, S.V. Pakhomov, and B.A. Volkov, Solid State Comm., 61, 2 93-96 (1987)

[85] M. König, S. Wiedmann, C. Brüne, A. Roth, H. Buhmann, L.W. Molenkamp, X-L. Qi, and S-C. Zhang, Science, 318, 766-770

[86] D. Hsieh, D. Qian, L. Wray, Y. Xia, Y.S. Hor, R.J. Cava, and M.Z. Hasan, Nature, 452, 970-974

[87] H. Zhang, C-X. Liu, X-L. Qi, X. Dai, Z. Fang, and S-C. Zhang, Nat. Phys., 5, 438-442 (2009)

[88] J.G. Analytis, J-H. Chu, Y. Chen, F. Corredor, R.D. McDonald, Z.X. Shen, and I.R. Fisher, Phys. Rev. B, 81, 205407 (2010)

[89] J.G. Checkelsky, Y.S. Hor, R.J. Cava, and N.P. Ong, Phys. Rev. Lett., 106, 196801 (2011) 
[90] Z. Ren, A.A. Taskin, S. Sasaki, K. Segawa, and Y. Ando, Phys. Rev. B, 82, 241306 (2010)

[91] C. Weyrich, M. Drögeler, J. Kampmeler, M. Eschbach, G. Mussler, T. Merzenich, T. Stoica, J. Schubert, L. Plucinski, B. Beschoten, C.M. Schnelder, C. Stampfer, D. Grützmacher, and Th. Schäpers, J. Phys. Cond. Matt., 28, 495501 (2016)

[92] Z. Ren, A.A. Taskin, S. Sasaki, K. Segawa, and Y. Ando, Phys. Rev. B, 84, 165311 (2011)

[93] A.A. Taskin, Z. Ren, S. Sasaki, K. Segawa, and Y. Ando, Phys. Rev. Lett., 107, 016801 (2011)

[94] T. Arakane, T. Sato, S. Souma, K. Kosaka, K. Nakayama, M. Komatsu, T. Tatahashi, Z. Ren, K. Segawa, and Y. Ando, Nat. Comm., 3, 636 (2012)

[95] T.P. Ginley, Y. Wang, and S. Law, Crystals, 6, 154 (2016)

[96] G. Zhang, H. Qin, J. Teng, J. Guo, Q. Gue, X. Dai, Z. Fang, and K. Wu, Appl. Phys. Lett., 95, 053114 (2009)

[97] J. Kellner, M. Eschbach, J. Kampmeier, M. Lanius, E. Mlynczak, G. Mussler, B. Holländer, L. Plucinski, M. Liebmann, D. Grützmacher, C.M. Schneider, and M. Morgenstern, Appl. Phys. Lett., 107, 251603 (2015)

[98] M. Eschbach, E. Mlynczak, J. Kellner, J. Kellner, J. Kampmeier, M. Lanius, E. Neumann, C. Weyrich, M. Gehlmann, P. Gospodaric, S. Döring, G. Mussler, N. Demarina, M. Luysberg, G. Bihlmayer, Th. Schäpers, L. Plucinski, S. Blügel, M. Morgenstern, C.M. Schneider, and D. Grützmacher, Nat. Comm., 6, 8816 (2015)

[99] M.Lanius, J. Kampmeier, G. Weyrich, S. Kölling, M. Schall, P. Schüffelgen, E. Neumann, M. Luysberg, G. Mussler, P.M. Koenraad, Th. Schäpers, and D. Grützmacher, Crys. Growth Des., 16, 2057-2061 (2016)

[100] C. Li, B. de Ronde, A. Nikitin, Y. Huang, M.S. Golden, A. de Visser, and A. Brinkman, Phys. Rev. B, 96, 195427 (2017)

[101] K. Vaklinova, A. Hoyer, M. Burgard, K. Kern, Nano. Lett. 16(4), 2595-2602 (2016) 
[102] W. Tremel, and R. Hoffmann, J. Am. Chem. Soc., 1987, 109 124-140 (1987)

[103] F. Jellinek, and H. Hahn, Naturwissenschaften, 5, 103 (1962)

[104] A.J. Klein Haneveld, F. Jellinek, Rec. Trav. Chim., 83, 776 (1964)

[105] H. Onken, K. Vierheilig, and H. Hagn, Zeitschrift für anorganische und allgemeine Chemie, 333, 4-6 (1964)

$[106]$

[107] L.M. Schoop, M.N. Ali, C. Strasser, A. Topp, A. Varykhalov, D. Marchenko, V. Duppel, S.S.P. Parkin, B.V. Lotsch, and C.R. Ast, Nat. Comm., 7, 11696 (2016)

[108] Y. Lv, B. Zhang, X. Li, S. Yao, Y.B. Chen, J. zhou, S. Zhang, M. Lu, and Y. Chen, Appl. Phys. Lett., 108, 244101 (2016)

[109] R. Sankar, G. Peramaiyan, I.P. Muthuselvam, C.J. Butler, K. Dimitri, M. Neupane, G.N. Rao, M.-T. Lin, and F.C. Chou, Sc. Rep., 7, 40603 (2017)

[110] M.N. Ali, L.M. Schoop, C. Garg, J.M. Lippmann, E. Lara, B. Lotsch, S.S.P. Parkin, Sc. Adv. 2, 160742

[111] S. Pezzini, M.R. van Delft, L.M. Schoop, B.V. Lotsch, A. Carrington, M.I. Katsnelson, N.E. Hussey, and S. Wiedmann, Nat. Phys., 14, 178-182 (2018)

[112] W.O.J. Groeneveld Meijer, American Mineralogist, 40, 646-657 (1955)

[113] F. Grønvold, and E. Røst, Acta Chemica Scand., 10, 1620-1634 (1956)

[114] J. Guggenheim, F. Hulliger, and J. Müller, Helv. Phys. Acta, 34, 408 (1961)

[115] Y. Liu, J. Zhao, L. Yu, C. Lin, A. Liang, C. Hu, Y. Ding, Y. Xu, S. He, L. Zhao, Chin. Phys. Lett., 32, 067303 (2015)

[116] H. Noh, J. Jeong, E. Cho, K. Kim, B.I. Min, B. Park, Phys. Rev. Lett., 119, 016401 (2017) 
[117] F. Fei, X. Bo, R. Wang, B. Wu, J. Jiang, D. fu, M. Gao, H. Zheng, Y. Chen, X. Wang, H. Bu, F. Song, X. Wang, B. Wang, and G. Wang, Phys. Rev. B, 96, 041201 (2017)

[118] H. Leng, C. Paulsen, Y.K. Huang, and A. de Visser, Phys. Rev. B, 96, 220506 (2017)

[119] S. Das, Amit, A. Sirohi, L. Yadav, S. Gayen, Y. Singh, and G. Sheet, Phys. Rev. B, 97, 014523 (2018)

[120] S. Teknowijoyo, N.H. Jo, M.S. Scheurer, M.A. Tanatar, K. Cho, S.L. Bud'ko, P.P. Orth, P.C. Canfield, and P. Prozorov, Phys. Rev. B, 98, 024508 (2018)

[121] A. Lyons, D. Schleich, A. Wold, Mat. Res. Bull., 11, 9 1155-1159 (1976)

[122] Y. Xia, D. Qian, D. Hsieh, L. Wray, A. Pal, H. Lin, A. Bansil, D. Grauer, Y.S. Hor, R.J. Cava, and M.Z. Hasan, Nat. Phys. 4, 398 (2009)

[123] D Hsieh, Y. Xia, D. Qian, L. Wray, J.H. Dil, F. Meier, J. Osterwalder, L. Patthey, J.G. Checkelsky, N.P. Ong, A.V. Fedorov, H. Lin, A. Bansil, D. Grauer, Y.S. Hor, R.J. Cava, and M.Z. Hasan, Nature 460, 1101 (2009)

[124] Z.-H. Pan, E. Vescovo, A.V. Fedorov, D. Gartner, Y.S. Lee, S. Chu, G.D. Gu, and T. Valla, Phys. Rev. Lett. 106, 257004 (2011)

[125] C. Jozwiak, Y.L. Chen, A.V. Fedorov, J.G. Analytis, C.R. Rotundu, A.K. Schmid, J.D. Denlinger, Y.-D. Chuang, D.-H. Lee, I.R. Fisher, R.J. Birgeneau, Z.-X. Shen, Z. Hussain, and A. Lanzara, Phys. Rev. B 84, 165113 (2011)

[126] C.H. Li, O.M.J. van 't Erve, Y.Y. Li, L. Li, and B.T. Jonker, Sci. Rep., 629533 (2016)

[127] Y. Ando, T. Hamasaki, T. Kurokawa, K. Ichiba, F. Yang, M. Novak, S. Sasaki, K. Segawa, Y. Ando, and M. Shiraishi, Nano. Lett. 14, 6226-6230 (2014)

[128] Y. Shiomi, K. Nomura, Y. Kajiwara, K. Eto, M. Novak, K. Segawa, Y. Ando, and E. Saitoh, Phys. Rev. Lett. 113, 196601 (2014) 
[129] A. Dankert, J. Geurs, M. Venkata Kamalakar, S. Charpentier, and S.P. Dash, Nano. Lett. 15, 7976-7981 (2015)

[130] J.S. Lee et al, Phys. Rev. B 92, 155312 (2015)

[131] C.H Li, O.M.J. van 't Erve, J.T. Robinson, Y. Liu, L. Li, and B.T. Jonker, Nat. Nano, 9 218-224 (2014)

[132] L. Liu, A. Richardella, I. Garate, Y. Zhu, N. Samarth, and C-T. Chen, Phys. Rev. B 91, 235437 (2015)

[133] J. Tang, L-T. Chang, X. Kou, K. Murata, E.S. Choi, M. Lang, Y. Fan, Y. Jiang, M. Montazeri, W. Jiang, Y. Wang, L. He, and K.L Wang, Nano Lett. 14, 5423-5429 (2014)

[134] E.K. de Vries, A.M. Kamerbeek, N. Koirala, M. Brahlek, M. Salehi, S. Oh, B.J. van Wees, and T. Banerjee, Phys. Rev. B 92, 201102(R) (2015)

[135] F. Yang, S. Ghatak, A.A. Taskin, K. Segawa, Y. Ando, M. Shiraishi, Y. Kanai, K. Matsumoto, A. Rosch, Y. Ando, Phys. Rev. B 94, 075304 (2016)

[136] K. Vaklinova, K. Polyudov, M. Burghard, and K. Kern, J. Phys.: Condens. Matter 30, 105302 (2018)

[137] J. Tian, I. Childres, H. Cao, T. Shen, I. Miotkowski, Y.P. Chen, Solid State Comm. 191, 1-5 (2014)

[138] Y. Wang, P. Deorani, K. Banerjee, N. Koirala, M. Brahlek, S. Oh, and H. Yang, Phys. Rev. Lett., 114, 257202 (2015)

[139] J. Tian, S. Hong, I. Miotowski, S. Datta, and Y.P. Chen, Sci. Adv. 3, e1602531 (2017)

[140] Y. Pan, D. Wu, J.R. Angevaare, H. Luigjes, E. Frantzeskakis, N. de Jong, E. van Heumen, T.V. Bay, B. Zwartsenberg, Y.K. Huang, M. Snelder, A. Brinkman, M.S. Golden, and A. de Visser, New Jour. Phys., 16123053 (2014)

[141] S. Hong, V. Diep, S. Datta, and Y.P. Chen, Phys. Rev. B 86, 085131 (2012)

[142] P. Lee and I. Applebaum, Phys. Rev. B., 93, 220404(R) (2016) 
[143] C. Li, B. de Ronde, A. Nikitin, Y. Huang, M.S. Golden, A. de Visser, and A. Brinkman, Phys. Rev. B, 96, 195427 (2017)

[144] X. Li, W. Cai, J. An, S. Kim, J. Nah, D. Yang, R. Piner, A. Velamakanni, I. Jung, E. Tutuc, S.K. Banerjee, L. Colombo, R.S. Ruoff, Science, 324, 1312-1314 (2009)

[145] L. Banszerus, M. Schmitz, S. Engels, J. Dauber, M. oellers, F. Haupt, K. Watanabe, T. Taniguchi, B. Beschoten, C. Stampfer Sci. Adv., 1 1500222, (2015)

[146] C. Józsa, T. Maassen, M. Popinciuc, P.J. Zomer, A. Veligura, H.T. Jonkman, and B.J. van Wees, Phys. Rev. B, 80, 241403(R) (2009)

[147] G. Stecklein, P.A. Crowell, J. Li, Y. Anugrah, Q. Su, and S.J. Koester, Phys. Rev. Appl., 6, 054015 (2016)

[148] O. Breunig, Z. Wang, A.A. Taskin, J. Lux, A. Rosch, and Y. Ando, Nat. Comm., 8, 15545 (2017)

[149] N. Borgwardt, J. Lux, I. Vergara, Z. Wang, A.A. Taskin, K. Segawa, P.H.M. van Loosdrecht, Y. ando, A. Rosch, and M. Grüniger, Phys. Rev. B, 93, 245149 (2016)

[150] L. Kimme, B. Rosenow, and A. Brataas, Phys. Rev. B, 93, 081301 (2016)

[151] A.M. Lunde, and G. Platero, Phys. Rev. B, 86, 035112 (2012)

[152] V. Cheianov, and L.I. Glazman, Phys. Rev. Lett., 110, 206803 (2013)

[153] Y. Tanaka, A. Furusaki, and K.A. Matveev, Phys. Rev. Lett., 106, $236402(2011)$

[154] A. Del Maestro, T. Hyart, and B. Rosenow, Phys. Rev. B, 87, 165440 (2013)

[155] S.A. Tarasenko, and G. Burkard, Phys. Rev. B, 94, 045309 (2016)

[156] A.M. Lunde, and G. Platero, Phys. Rev. B, 88115411 (2013)

[157] C. Hsu, P. Stano, J. Klinovaja, and D. Loss, Phys. Rev. B, 96, 081405 (2017) 
[158] C. Hsu, P. Stano, J. Klinovaja, and D. Loss, Phys. Rev. B, 97, 125432 (2018)

[159] J. Tian, S. Hong, I. Miotowski, S. Datta, and Y.P. Chen, Sci. Adv. 3, e1602531 (2017)

[160] A. Mert Bozkurt, B. Pekerten, and İ. Adagideli, Phys. Rev. B, 97, 245414 (2018)

[161] A.K. Koh, and D.J. Miller, Atomic data and nuclear data tables, 33, 235-253 (1985)

[162] D. Teweldebrhan, V. Goyal, and A.A. Balandin, Nano Lett., 10, 1209$1218(2010)$

[163] I. Adigadeli, private communications

[164] A.A. Burkov, M.D. Hook, and L. Balents, Phys. Rev. B, 84, 235126 (2011)

[165] T. Bzdušek, Q. Wu, A. Rüegg, M. Sigrist, and A.A. Soluyanov, Nature, 538, 75-78 (2016)

[166] C. Fang, H. weng, X. Dai, and Z. Fang, Chin. Phys. B, 25, 117106 (2016)

[167] Y. Chen, Y. Xie, S.A. Yang, H. Pan, F. Zhang, M.L. Cohen, and S. Zhang, Nano lett., 15, 6974-6978 (2015)

[168] S-Y. Yang, H. Yang, E. Derunova, S.S.P. Parkin, B. Yan, and M.N. Ali, Adv. in Phys. X, 3:1, 1414631 (2018)

[169] X. Wang, X. Pan, M. Gao, J. Yu, J. Jiang, J. Zhang, H. Zuo, M. Zhang, W. Niu, Z. Xia, X. Wan, Y. Chen, F. Song, Y. Xu, B. Wang, G. Wang, and R. Zhang, Adv. Electron. Mater., 2, 1600228 (2016)

[170] J. Zhang, M. Gao, J. Zhang, X. Wang, X. Zhang, M. Zhang, W. Niu, R. Zhang, Y. Xu, Front. Phys. 13, 137201 (2018)

[171] R. Singha, A.K. Pariari, B. Satpati, and P. Mandal, Proc. Nat. Acad. Sc., 1618004114 (2016)

[172] J. Hu, Z. Tang, J. Liu, X. Liu, Y. Zhu, D. Graf, K. Myhro, S. Tran, C.N. Lau, J. wei, and Z. Mao, Phys. Rev. Lett., 117, 016602 (2016) 
[173] A. Topp, R. Queiroz, A. Grüneis, L. Müchler, A.W. Rost, A. Varykhalov, D. Marchenko, M. Krivenkov, F. Rodolakis, J.L. McChesney, B.V. Lotsch, L.M. Schoop, and C.R. Ast, Phys. Rev. $\mathrm{X}, \mathbf{7}, 041073(2017)$

[174] A.N. Rudenko, E.A. Stepanov, A.I. Lichtenstein, and M.I. Katsnelson, Phys. Rev. Lett., 120, 216401 (2018)

[175] M. Matusiak, J.R. Cooper, and D. Kaczorowski, Nat. Comm., 8, $152192017)$

[176] J. Hu, Z. Tang, J. Liu, Y. Zhu, J. Wei, and Z. Mao, Phys. Rev. B., 96, 045127 (2017)

[177] C. Li, C.M. Wang, B. Wan, X. Wan, H-Z. Lu, and X.C. Xie, Phys. Rev. Lett., 120, 146602 (2018)

[178] S.I. Hyun, I. Lee, G. Lee, and J.H. Shim, Phys. Rev. B, 98, 165108 (2018)

[179] M.V. Berry, Proc. R. Soc. Lond. A, 392, 45-57 (1948)

[180] D. Xiao, M. Chang, and Q. Niu, Rev. Mod. Phys., 82, 1959 (2010)

[181] Y. Lv, X. Li, J. Zhang, B. Pang, S. Chen, L. Cao, B. Zhang, D. Lin, Y.B. Chen, S. Yao, J. Zhou, S. Zhang, M. Lu, M. Tian, and Y. Chen, Phys. Rev. B, 97, 245151 (2018)

[182] M. Matin, R. Mondal, N. Barman, A. Thamizhavel, and S.K. Dhar, Phys. Rev. B, 97, 205130 (2018)

[183] Y. Wang, K. Wang, J. Reutt-Robey, J. Paglione, and M.S. Fuhrer, Phys. Rev. B, 93, 121108 (2016)

[184] D. Rhodes, S. Das, Q.R. Zhang, B. Zeng, N.R. Pradhan, N. Kikugawa, E. Manousakis, and L. Balicas, Phys. Rev. B, 92, 125152 (2015)

[185] T. Liang, Q. Gibson, M.N. Ali, M. Liu, R.J. Cava, and N.P. Ong, Nat. Mater., 14, 280-284 (2015)

[186] L. Onsager, Phil. Mag., 43, 1006 (1952)

[187] I.M. Lifshitz, and A.M. Kosevich, Sov. Phys. JETP, 2, 636 (1956) 
[188] J. Hu, Z. Tang, J. Liu, X. Liu, Y. Zhu, D. Graf, J. Myhro, S. Tran, C.N. Lau, J. Wei, and Z. Mao, Phys. Rev. Lett., 117, 016602 (2016)

[189] C. Li, C.M. Wang, B. Wan, X. Wan, H. Lu, and X.C. Xie, Phys. Rev. Lett., 120, 146602 (2018)

[190] W.J. de Haas, and P.M. van Alphen, Proc. Acad. Sci. Amst., 33, 1106-1118 (1930)

[191] D. Schoenberg, Magnetic oscillations in metals (1984)

[192] L. Fu, and C.L Kane, Phys. Rev. Lett. 100, 096407 (2008)

[193] V. Mourik, K. Zuo, S.M. Frolov, S.R. Plissard, E.P.A.M. Bakkers, and L.P. Kouwenhoven, Science 336, 6084 pp 1003-1007 (2012)

[194] L.P. Rokhinson, X. Liu, and J.K. Furdyna, Nat. Phys. 8, 795-799 (2012)

[195] J. Wiedenmann, E. Bocquillon, R.S. Deacon, S. Hartinger, O. Herrmann, T.M. Klapwijk, L. Maier, C. Ames, C. Brüne, C. Gould, A. Oiwa, K. Ishibashi, S. Tarucha, H. Buhmann, and L.W. Molenkamp, Nat. Comm. 7, 10303 (2016)

[196] H-H. Sun, K-W. Zhang, L-H. Hu, C. Li, G-Y. Wang, H-Y. Ma, ZA. Xu, C-L. Gao, D-D. Guan, Y-Y. Li, C. Liu, D. Qian, Y. Zhou, L. Fu, S-C. Li, F-C. Zhang, and J-F. Jia, Phys. Rev. Lett. 116, 257003

[197] M. Snelder, M. Veldhorst, A.A. Golubov, and A. Brinkman, Phys. Rev. B 87, 104507 (2013)

[198] C. Li, J.C. de Boer, B. de Ronde, S.V. Ramankutty, E. van Heumen, Y. Huang, A. de Visser, A.A. Golubov, M.S. Golden, A. Brinkman, Nat. Mater. 17, 875-880 (2018)

[199] S. Sasaki, M. Kriener, K. Segawa, K. Yada, Y. Tanaka, M. Sato, and Y. Ando, Phys. Rev. Lett. 107, 217001 (2011)

[200] L. Fu, and E. Berg, Phys. Rev. Lett. 105, 097001 (2010)

[201] H. Leng, C. Paulsen, Y.K. Huang, and A. de Visser, Phys. Rev. B 96, 220506(R) (2017)

[202] Amit, and Y. Singh, Phys. Rev. B 97, 054515 (2018) 
[203] O.J. Clark, M.J. Neat, K. Okawa, L. Bawden, I. Marković, F. Mazzola, J. Feng, V. Sunko, J.M. Riley, W. Meevasana, J. Fujii, I. Vobornik, T.K. Kim, M. Hoesch, T. Sasagawa, P. Wahl, M.S. Bahramy, and P.D.C. King, Phys. Rev. Lett. 120, 156401 (2018)

[204] H-J. Noh, J Jeong, E-J. Cho, K. Kim, B.I. Min, B-G. Park, Phys. Rev. Lett. 119, 016401 (2017)

[205] Y. Liu, J. Zhao, L Yu, C. Lin, C. Hu, D. Liu, Y. Peng, Z. Xie, J. He, C. Chen, Y. feng, H. Yi, X. Liu, L. Zhao, S. He, G. Liu, X. Dong, J. Zhang, C. Chen, Z. Xu, H. Weng, X. Dai, Z. Fang, and X.J. Zhou, Chin. Phys. B 24, 067401 (2015)

[206] G.E. Blonder, M. Tinkham, and T.M. Klapwijk, Phys. Rev. B 25, 4515 (1982)

[207] G. Sheet, S. Mukhopadhyay, and P. Raychaudhuri, Phys. Rev. B 69, 134507 (2004)

[208] V. Baltz, A.D. Naylor, K.M. Seemann, W. Elder, S. Sheen, K. Westerholt, H. Zabel, G. Burnell, C.H. Marrows, and B.J. Hickey, J. Phys. Condens. Matter 21, 095701 (20096)

[209] D. Daghero, and R.S. Gonnelli, Supercond. Sci. Technol. 23, 043001 (2010)

[210] Yu.G. Naidyuk, and K. Gloos, Low Temp. Phys. 44, 257 (2018)

[211] P.S. Westbrook, and A. Javan, Phys. Rev. B 59, 14606 (1999)

[212] R. Taboryski, T. Clausen, J. Bindslev Hansen, J.L. Skov, J. Kutchinsky, C.B. Sørensen, and P.E. Lindelof, Appl. Phys. Lett. 69, 656 (1996)

[213] P. Raychaudhuri, D. Jaiswal-Nagar, G. Shout, S. Ramakrishnan, and H. Takeya, Phys. Rev. Lett., 93, 156802 (2004) (Supp. Mat.)

[214] D.L. Bashlakov, Yu.G. Naidyuk, I.K. Yanson, S.C. Wimbush, B. Holzapfel, G. Fusch, and S-L. Drechsler, Supercond. Sci. Tech., 18, 1094-1099 (2005)

[215] C. Nguyen, H. Kroemer, and E.L. Hu, Phys. Rev. Lett. 69, 2847 (1992) 
[216] H. Zhang, C-X. Liu, S. Gazibegovic, D. Xu, J.A. Logan, G. Wang, N. van Loo, J.D.S. Bommer, M.W.A. de Moor, D. Car, R.L.M. op het Veld, P.J. van Veldhoven, S. Koelling, M.A. Verheijen, M. Pendharkar, D.J. Pennachio, B. Shojaei, J.S. Lee, C.J.Palmstrøm, E.P.A.M. Bakkers, S. Das Sarma, and L.P. Kouwenhoven, Nature 556, $26142(2018)$

[217] K. Matano, M. Kriener, K. Segawa, Y. Ando, and G. Zheng, Nat. Phys. 12, 3781 (2016)

[218] T. Hashimoto, K. Yada, A. Yamakage, M. Sato, and Y. Tanaka, J. Phys. Soc. Jpn. 82, 044704 (2013)

[219] S. Yonezawa, K. Tajiri, S. Nakata, Y. Nagai, Z. Wang, K. Segawa, Y. Ando, and Y. Maeno, Nat. Phys. 13, 3907 (2017) 